Aus der Abteilung Prothetik

(Komm. Leiter: PD Dr. med. dent. N. Gersdorff)

im Zentrum Zahn-, Mund- und Kieferheilkunde

der Medizinischen Fakultät der Universität Göttingen

\title{
Vergleichende Analyse der Research Diagnostic Criteria for Temporomandibular Disorders (RDC/TMD) und der real-time- Magnetresonanztomographie
}

\section{Inaugural - Dissertation}

zur Erlangung des Doktorgrades für Zahnheilkunde

der Medizinischen Fakultät der Georg-August-Universität zu Göttingen

vorgelegt von

Olaf Ansgar Chrysanthus Kling

aus

Bonn 
Dekan:

Prof. Dr. med. C. Frömmel

I. Berichterstatter: PD. Dr. med. dent. N. Gersdorff

II. Berichterstatter/in:

II. Berichterstatter/in:

Tag der mündlichen Prüfung: 


\section{$\underline{\text { Inhaltsverzeichnis }}$}

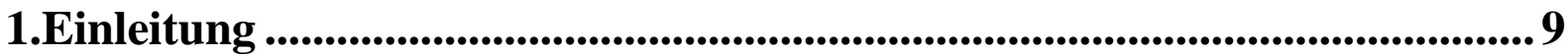

1.1. Anatomischer Aufbau und Funktion des Kiefergelenks ................................................... 11

1.2 Pathophysiologie der Kraniomandibulären Dysfunktion (CMD) ......................................... 16

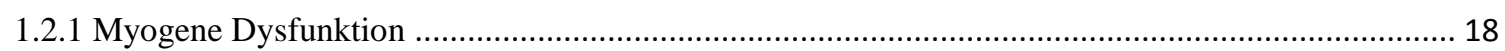

1.2.1.1Pathophysiologie der myogenen Dysfunktion ................................................................... 18

1.2.1.2 Symptomatik der myogenen Dysfunktion ..................................................................... 19

1.2.1.3 Therapie der myogenen Dysfunktion .................................................................................. 20

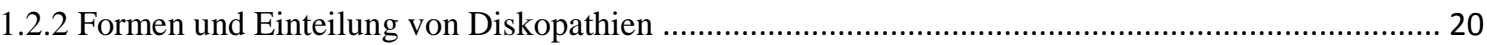

1.2.2.1 Pathophysiologie der Diskopathien .................................................................................. 20

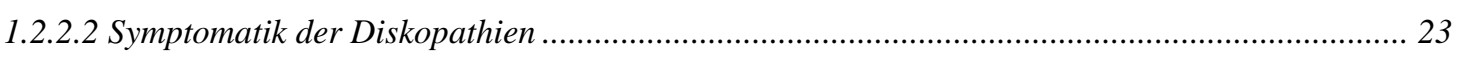

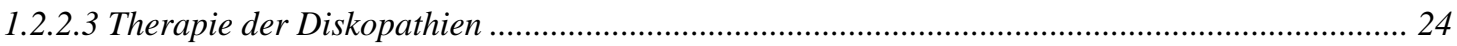

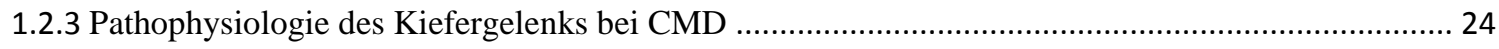

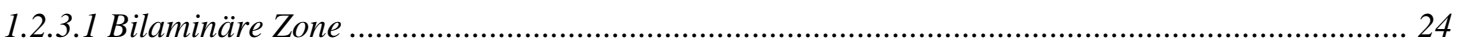

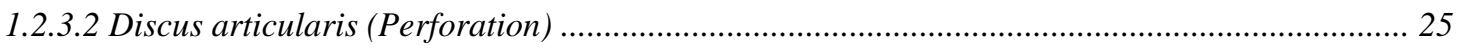

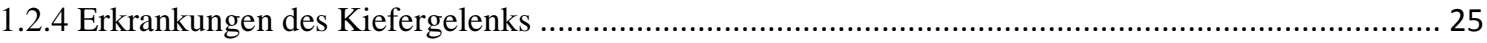

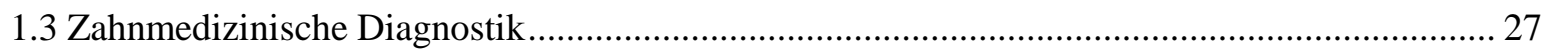

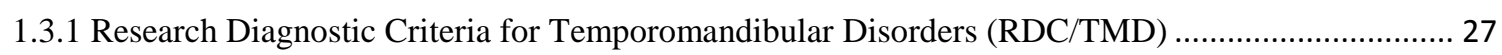

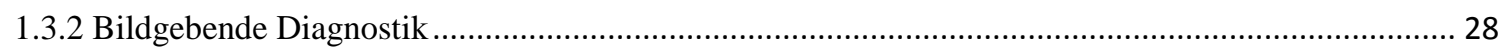

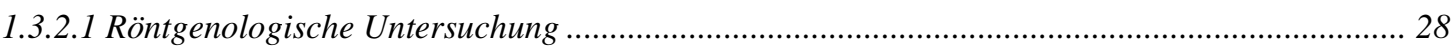

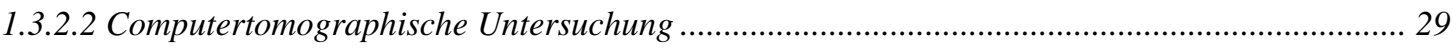

1.3.2.3 Magnetresonanztomographische Untersuchung ................................................................. 29

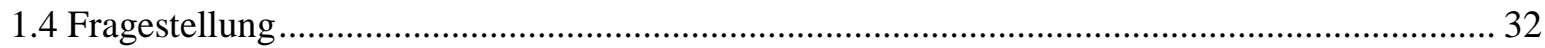

2. Material und Methoden ...........................................................................................33

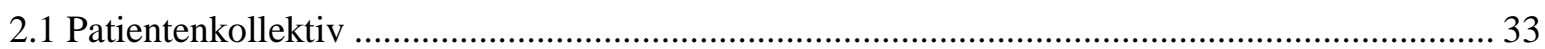




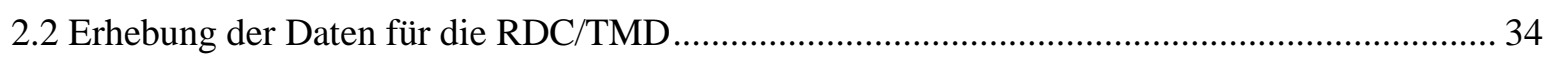

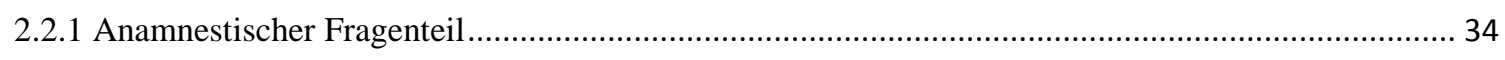

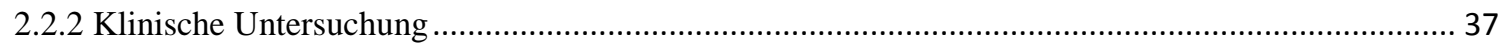

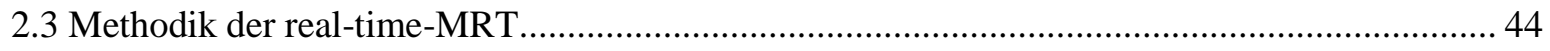

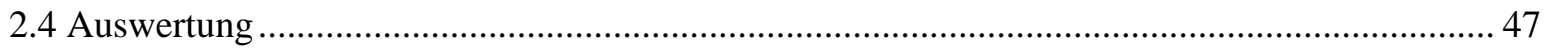

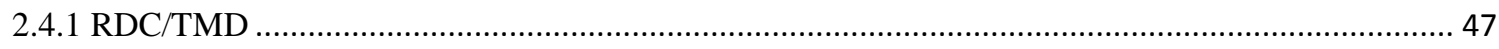

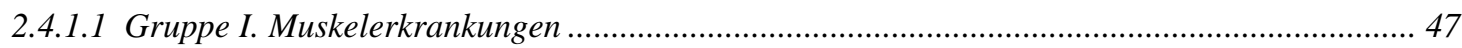

2.4.1.2. Gruppe II. Verlagerung des Discus articularis............................................................... 49

2.4.1.3. Gruppe III. Arthralgie, Arthritis, Arthrose des Kiefergelenks ........................................... 51

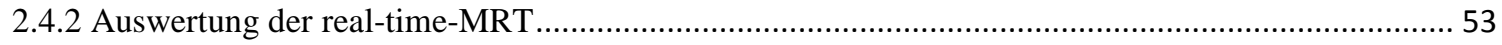

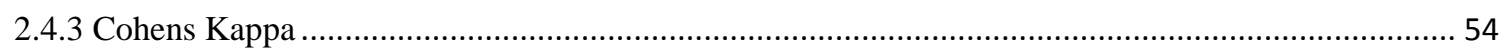

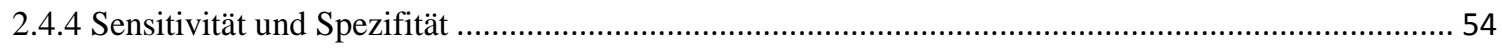

3. Ergebnisse ….........................................................................................................................56

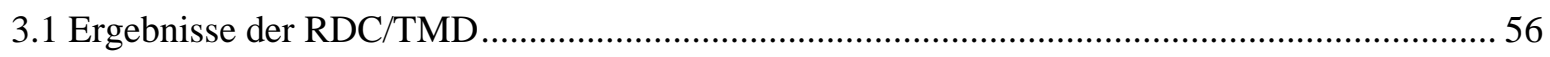

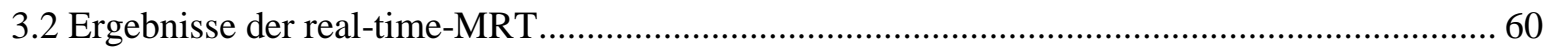

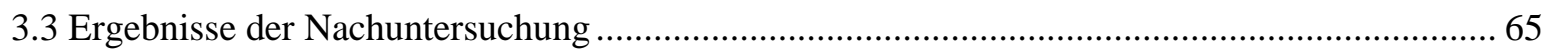

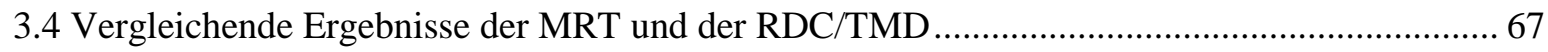

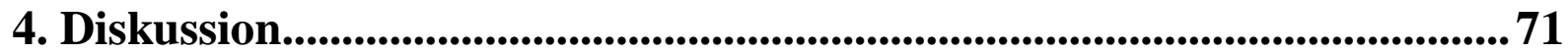

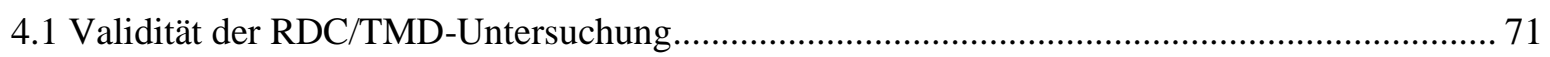

4.2 Validität der real-time-MRT gegenüber der statischen MRT …............................................. 77

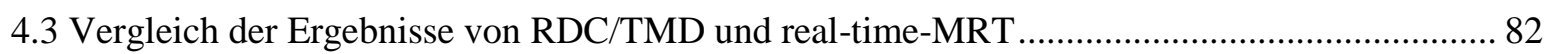

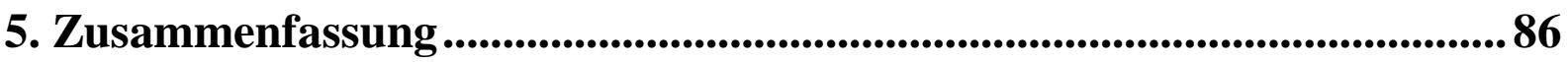




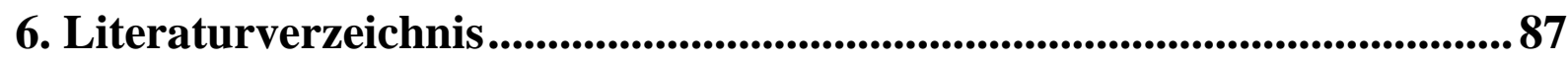

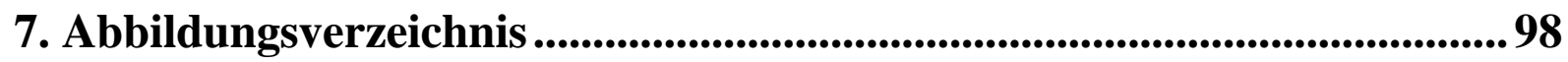

8. Tabellen-/Diagrammverzeichnis ..............................................................99

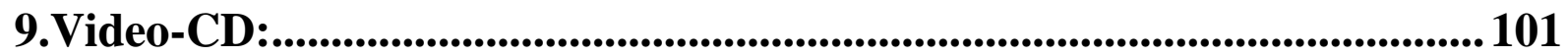




\section{Abkürzungsverzeichnis}

A.H.

Abb.

ATP

CBCT

CINE-Mode

CMD

CT

2D-FLASH-Sequenz

DGZMK

diff.

DV

DVR

entspr.

et al

F.

FOV

Gadolinium-DTPA

i.v.

$\kappa$

$\mathrm{kDV}$

li.

Lig.

lim.

M.

M.W.

Max.

$\mathrm{mm}$

MMP

MRT

N.G.

NMR-Forschungs GmbH

O.K.
Prof. Dr. Dr. Alfons Hüls

Abbildung

Adenosin-Triphosphat

Cone Beam Computed Tomography

Kinematographischer Modus

Kraniomandibuläre Dysfunktion

Computertomographie

2 dimensionale fast low angle shot Sequenz

Deutsche Gesellschaft für Zahn-, Mund- und Kieferkrankheiten

Differenz

Diskusverlagerung

Diskusverlagerung ohne Reposition

entsprechend

et alii

Frage

Field of view

Gadolinium Diethylenetriaminopentaacetic Acid

intravenös

Kappa

Keine Diskusverlagerung

links

Ligamentum

limitierte

Musculus

Dr. M. Wasser

Maximale

Millimeter

Matrix-Metalloproteinasen

Magnetresonanztomographie

PD Dr. Nikolaus Gersdorff

nuklearmagnetische Resonanz-Forschungs Gesellschaft mit beschränkter Haftung

ZA Olaf Kling 
OPG

Pass.

Proc.

RDC/TMD

re.

sec.

sog.

STR

$\mathrm{T}$

Tab.

TE

TNF $\alpha$

TR

v.D.

VEGF

z. B.
Orthopantomogramm

passive

Processus

Research Diagnostic Criteria for Temporomandibular Disorders rechts

Sekunden

sogenannte

Stretch

Tesla

Tabelle

Gradient Echo Time

Tumor-Nekrose-Faktor $\alpha$

Repetition Time

verlagerter Diskus

endothelialer Wachstumsfaktor

zum Beispiel 


\section{Einleitung}

In der heutigen Zeit begegnen den Zahnärzten auch im klinischen Alltag immer häufiger Kiefergelenksprobleme. Neben den arrivierten Tätigkeitsfeldern eines Zahnarztes ist daher der Diagnostik und Behandlung von pathophysiologischen Veränderungen im Kiefergelenk größte Aufmerksamkeit zu widmen. Dabei steht trotz der Vielzahl an Ursachen und Symptomen für Kiefergelenksprobleme im Vordergrund, mit Hilfe von effizienten Untersuchungsmethoden und Untersuchungsverfahren die für jeden Patienten individuell adäquateste Behandlung einleiten zu können.

In der heutigen internationalen Literatur stehen für dasselbe Syndrom verschiedene Begriffe zur Verfügung, wie zum Beispiel Internal Derangement (Katzberg et al. 1996), Myoarthropathie (Türp und Hugger 2000), myofaziales Schmerzdysfunktionssyndrom, temporomandibuläre Funktionsstörungen (Carlson und Magnusson 1999), Temporomandibular Disorders (McNeill 1997), Costen Syndrom (Costen 1997) oder Craniofacial Dysfunction (Krestan et al. 2001).

Im weiteren Textverlauf wird ausschließlich der von der DGZMK verwendete Begriff „Craniomandibuläre Dysfunktion“ bzw. die aus der englischen Schreibweise abgeleitete Abkürzung „CMD“ (craniomandibular disorders) verwendet.

Der Ausdruck CMD ist somit ein Sammelbegriff für ein breites Spektrum an klinischen Gelenk- und Muskelproblemen im orofazialen Bereich. CMDs gelten als spezielle Untergruppe von muskuloskelettalen und rheumatologischen Störungen; sie stellen die Hauptursache für nicht dentale Schmerzen im orofazialen System dar (Carlson und Magnusson 1999).

Als Leitsymptome dieser Erkrankungen sind Bewegungseinschränkungen des Unterkiefers (Limitation), Schmerzen im Kiefergelenksbereich, Kiefergelenksgeräusche, Druckdolenzen in der Kaumuskulatur als auch Deviationen zu nennen. Die große Anzahl von Symptomen kommt vor allem dadurch zustande, dass nicht immer alle Symptome, die theoretisch möglich wären, auch gleichzeitig auftreten. Daher kann man von einem multikausalen Erscheinungsbild sprechen.

Es hat sich bei epidemiologischen Untersuchungen von Bevölkerungsgruppen gezeigt, dass bei bis zu 75\% der Bevölkerung mindestens ein Anzeichen bzw. ein Symptom einer CMD vorhanden ist. Allerdings bleiben diese von der Bevölkerung oft unbemerkt oder werden tole- 
riert, da sie keinerlei Schmerzen verursachen. So kommt es dazu, dass nur 5 - 7\% der Bevölkerung wegen einer durch CMD verursachten Problematik behandelt werden müssen (McNeill 1997). Dabei sind Frauen ungefähr doppelt so häufig von einer schmerzhaften CMD betroffen wie Männer (Carlson und Magnusson 1999; Türp und Hugger 2000). Am häufigsten treten auf CMD zurückzuführende Symptome bei Personen zwischen der zweiten und vierten Lebensdekade auf und nehmen mit zunehmendem Alter ab. Da der Krankheitsverlauf der CMD sich häufig als sehr langwierig und komplex darstellt, ist die CMD verantwortlich dafür, dass auch im zahnärztlichen Bereich immer wieder mit dem Beschwerdebild: „,chronischer Schmerzpatient“ zu rechnen ist. Diese spezielle Patientenklientel ist zudem mit wesentlichen physischen und psychosozialen Beeinträchtigungen belastet, wobei auch die gesundheitsökonomischen Auswirkungen für die Gesellschaft nicht außer Acht gelassen werden dürfen (Katzberg et al. 1996; McNeill 1997; Türp und Hugger 2000).

Da klinische Untersuchungen nur begrenzt in der Lage sind, spezifische Diagnosen zu stellen und zu differenzieren, und diese überdies stark mit den Erfahrungen des Behandlers variieren können, sollten bildgebende Verfahren bei der Therapieentscheidung immer mit berücksichtigt werden. Als Standard für die Darstellung der knöchernen Kiefergelenksstrukturen gilt das Orthopantomogramm (OPG) bzw. die Computertomographie (CT). Zur Überprüfung der klinischen Befunde hat sich insbesondere bei Verdacht auf eine Diskusverlagerung die Magnetresonanztomographie (MRT) bewährt. Die MRT ist als nicht-invasives bildgebendes Verfahren in der Lage, sowohl die Position des Diskus als auch die Morphologie der knorpeligen und weichgewebigen Kiefergelenksstrukturen mit großer Zuverlässigkeit darzustellen (Westesson 1993; Krestan et al. 2001). Inzwischen ist sie durch stetige Weiterentwicklung zum „Goldstandard“ in der Kiefergelenksdiagnostik avanciert und hilft dabei, die Diagnose und Therapieentscheidung zu erleichtern (Krestan et al. 2001). 


\subsection{Anatomischer Aufbau und Funktion des Kiefergelenks}

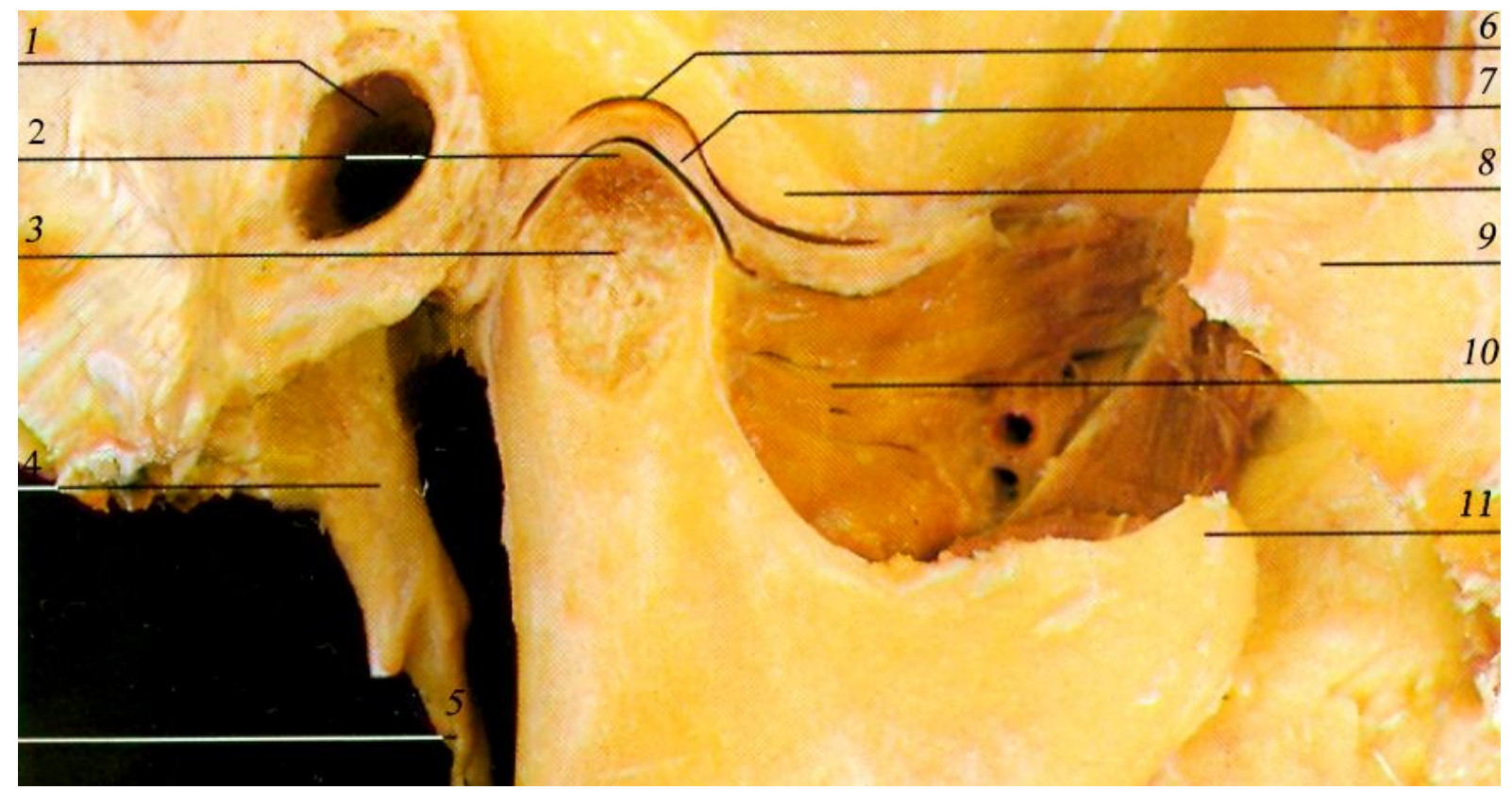

Abb. 1 Anatomie des Kiefergelenks 1. Gehörgang (= Porus acusticus) 2. Cartilago articularis proc. condylaris 3. Kondylus ( = Caput mandibulae, $=$ Processus condylaris) 4. Proc. Styloideus 5. Lig. Stylomandibulare 6. Fossa mandibularis (= Fossa articularis) 7. Discus articularis 8. Eminentia (= Tuberculum articulare, = Gelenkhöcker, = Protuberantia articularis) 9. Os zygomaticum 10. Musculus pterygoideus lateralis 11. Proc. coronideus (modifiziert Rohen et al. 2002 S. 56)

Die funktionelle Verbindung des Unterkiefers (Mandibula) mit dem Schläfenbein (Os temporale) wird durch das Kiefergelenk (Articulatio temporomandibularis) (Abb. 1) dargestellt.

Der Unterkiefer stellt mit dem Kondylus einen Teil des Gelenks dar, der durch den Diskus (Discus articularis) von der Gelenkfläche am Os temporale, dem anderen Teil des Gelenkes, getrennt ist.

Der einer abgerundeten Walze ähnelnde Unterkieferkopf (Caput mandibulae, Kondylus) (Abb.1 Punkt 3) sitzt antevertiert auf dem Collum mandibulae, einem Teil des Processus condylaris. Dabei zeigen die Längsachsen der beiden Kieferköpfe ein wenig schräg nach dorsal und medial. Ihre Achsen schneiden sich dabei vor dem Foramen magnum in einem Winkel von $150-165^{\circ}$. Der Gelenkkopf ist vom anterioren bis zum posterioren Rand des Gelenks mit Faserknorpel überzogen ebensowie der anteriore Teil der Fossa mandibularis (Abb.1 Punkt 6). Er reicht bis an die Fissura petrotympanica und bildet die Facies temporalis. Der posteriore Teil liegt extrakapsulär. Dort verläuft am Rand ein Nerv, die Chorda tympani. Der Gelenkraum ist immer noch so groß konzepiert, dass sich der Unterkieferkopf bei Mahlbewegungen, also bei einer Drehung um die vertikale Achse, schräg stellen kann. Nach anterior greift die Facies articularis auf das Tuberculum articulare (Abb.1 Punkt 3) über, das ebenso wie der anteriore Abschnitt der Fossa mandibularis (Abb.1 Punkt 6) mit Faserknorpel überzogen ist. Dieser Gelenkhöcker bildet eine querstehende Rolle, die von anterior nach posterior konvex 
gekrümmt ist und an deren posterioren Fläche sich der Unterkieferkopf in der Ruhelage unter Zwischenschaltung des Diskus (Abb.1 Punkt 7) anlagert (Benninghoff und Drenckhahn 2003).

Der Discus articularis (Abb.1 Punkt 7) besteht aus unterschiedlichen Gewebearten. Die dünne, in der Mitte liegende intermediäre Zone ist aus straffem Bindegewebe aufgebaut, während die verdickten Randzonen aus Faserknorpel bestehen. Diese Verdickungen heften sich ringsum an die Gelenkkapsel und zerlegen das Gelenk in zwei Kammern. Der Diskus bedeckt kappenartig den Kieferkopf und begleitet ihn als bewegliche Pfanne bei seinen Verschiebungen. Gleichzeitig bildet der Diskus mit seiner oberen Fläche eine Pfanne für den Gelenkhöcker. Dieser ist medial und lateral mit der Gelenkkapsel verwachsen und löst sich nach hinten in einen bindegewebigen, reichlich elastische Fasern enthaltenden anatomisch begrenzten Raum - die bilaminäre Zone - auf (Parsons und Boucher 1966; Benninghoff und Drenckhahn 2003). Dieser besteht aus einem Stratum superius und einem Stratum inferius. Zwischen beiden liegt das Genu vasculosum mit zahlreichen Gefäßen, Nerven und Fett. Das Stratum superius ist aus einem lockeren Netzwerk aus elastischen und kollagenen Fasern, Fett und Gefäßen aufgebaut. Im Gegensatz dazu besteht das Stratum inferius aus straffen, kollagenen Fasern. In der bilaminären Zone sind die kollagenen Fasern locker organisiert und verlaufen annähernd sagittal. Die Fasern beider Strata strahlen in die Pars posterior des Diskus ein und vernetzen sich dort mit den transversalen Fasern der Pars posterior und mit den sagittalen Fasern der Pars intermedia. Die elastischen Fasern in der bilaminären Zone weisen einen größeren Durchmesser als im Diskus auf und sind vorwiegend im Stratum superius konzentriert. Das Stratum superius ist dorsal mit dem Processus glenoidalis am knöchernden Gehörgang, am knorpeligen Teil des Gehörgangs und an der Faszie der Ohrspeicheldrüse (Glandula parotidea) fixiert (Parsons und Boucher 1966; Bumann und Lotzmann 2000).

Hinzu kommt, dass in den Discus articularis Fasern des M. pterygoideus lateralis von anterior einstrahlen und er an seiner medialen Seite mit der Oberseite des Muskels verheftet ist. Der gesamte M. pterygoideus lateralis kontrolliert die Bewegungen, die im Kiefergelenk ablaufen. Mittlere und laterale Abschnitte des Discus articularis werden außerdem noch von Fasern des M. masseter und M. temporalis erreicht.

Die Gelenkkapsel ist so weit, dass der Kieferkopf nach anterior vor das Tuberculum articulare luxieren kann. Sie umfasst die Gelenkhöcker, heftet sich seitlich an den Rand der Gelenkpfanne und reicht bis zur Fissura petrotympanica. Die Fasern konvergieren trichterförmig zum Kieferkopf, an dem die Kapsel am posterioren Ende weiter herab reicht als am anterioren. Eine gewisse Verstärkung erfährt die seitliche Kapselwand durch das Lig. laterale. Zwei wei- 
tere Ligamente, die zwar am Kiefergelenk selbst beteiligt, aber keine Beziehung zur Gelenkkapsel haben, sind zum einen das Lig. sphenomandibulare und zum anderen das Lig. stylomandibulare (Benninghoff und Drenckhahn 2003).

Bei der physiologischen Mundöffnung wird durch die suprahyoidale Muskulatur eine initiale Rotation und durch den M. pterygoideus lateralis die sich anschließende Translation ermöglicht. Dabei führen Rotationsbewegungen des Kondylus stets zu einer Relativbewegung des Diskus nach dorsal und somit zu einer Stabilisierung des Diskus auf dem Kondylus. Schematisch werden alle Bewegungen des Inzisalpunktes in der Sagittalen, häufig im sog. PosseltDiagramm dargestellt. Dabei stellen die hier blauen und roten Pfeile die habituelle Mundöffnungs- und Mundschließbewegung dar. Alle anderen Punkte und Linien sind als Grenzpunkte und Grenzbewegungen der Protrusion, Retrusion und Laterotrusion sowie der Mundöffnung und des Mundschlusses zu verstehen.

Mundöffnungsbewegung (Abb. 2):
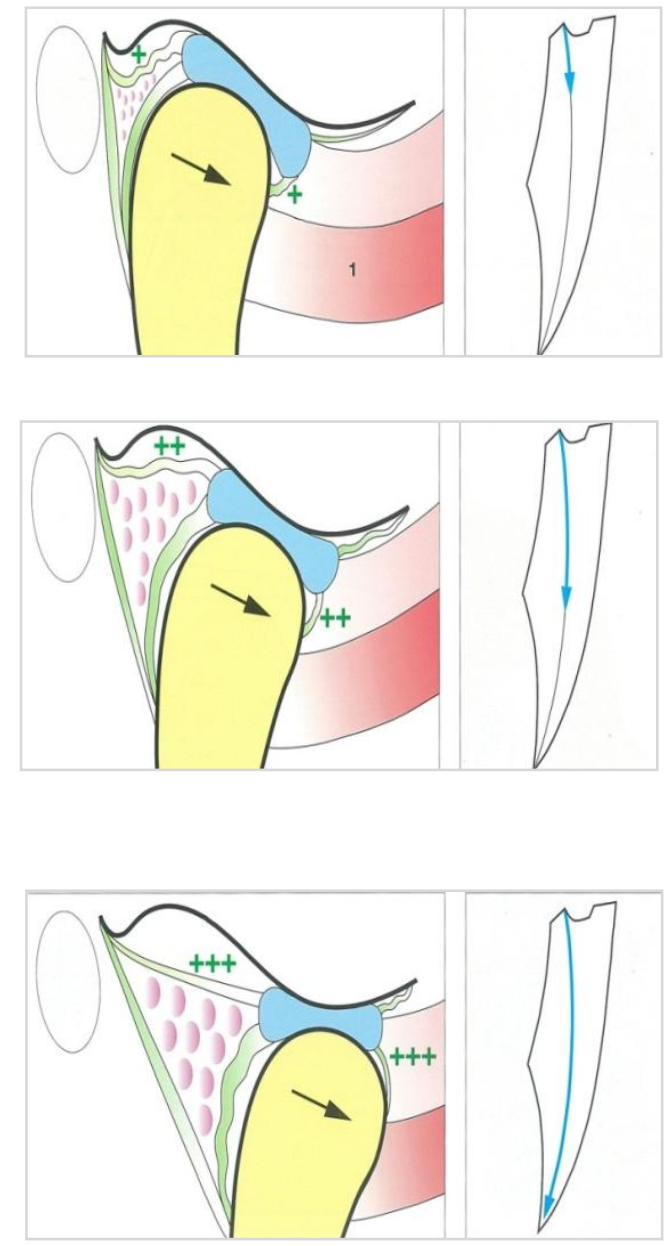

Abbildung 2: „Bewegungsmuster Mundöffnung 1. Initiale Phase: Schematische Darstellung der Belastungen während der initialen Mundöffnung. Der Kondylus führt eine Rotationsbewegung aus. Dadurch verändert sich die Diskusposition in Relation zur Fossa nur unwesentlich. So bewegt sich der Diskus nach dorsal in Relation zum Kondylus. Die elastischen Fasern werden nur geringfügig aus ihrem Äquilibrium gebracht. Vom M. pterygoideus lateralis ist nur das Caput inferius (1) aktiv (Abb. re.: entspr. Posselt-Diagramm).

2. Intermediäre Phase: Der Kondylus vollzieht eine deutliche Translation. Dies führt zu einer ventralen Bewegung des Diskus in Relation zur Fossa und zu einer dorsalen Bewegung in Relation zum Kondylus. Der Spannungszustand im Stratum superius und in der vorderen Gelenkkapsel nimmt stetig zu. Das Startum inferius entspannt. Das Genu vasculosum expandiert und entwickelt einen Unterdruck, so dass es sich mit Blut füllt (Abb. re.: entspr. PosseltDiagramm).

3. Terminale Phase: Der Kondylus erreicht das größte Ausmaß an Rotation und Translation. Die Translationskomponente führt den Diskus weiter passiv nach ventral. Die Rotation fördert weiterhin die Relativbewegung nach dorsal. Das Stratum superius und die untere Gelenkkapsel sind jetzt maximal gespannt. Der retrokondyläre Raum ist durch das in das Genu vasculosum einströmende Blut ausgefüllt. Das Stratum inferius ist entspannt (Abb. re.: entspr. PosseltDiagramm)“ (Bumann und Lotzmann 2000 S. 46). 
Während der Translation wird der Diskus passiv nach ventral mitbewegt. Mit der Mundöffnung steigt die Spannung im Stratum superius und der vorderen Gelenkkapsel. Die Mundöffnung wird durch die Gelenkkapsel und das Lig. laterale begrenzt, nicht jedoch vom Stratum superius. Dieses begrenzt lediglich die passive ventrale Bewegung des Diskus. Das Genu vasculosum wird auf das vier- bis fünfache seines Ausgangszustandes gedehnt und weist einen Unterdruck auf.

Bei der physiologischen Mundschließung werden der M. temporalis, der M. masseter, der M. pterygoideus medialis und das Caput superius des M. pterygoideus lateralis aktiviert. Bei der Schließbewegung gilt, dass der Diskus eine Relativbewegung - in Relation zum Kondylus nach ventral durchführt. Während der Kondylus muskulär nach dorsal in die Fossa gezogen wird, führen mehrere Strukturen den Diskus nach dorsal und verhindern am Ende der Schließbewegung eine anteriore Vorverlagerung des Diskus.

Mundschließbewegung (Abb.3):
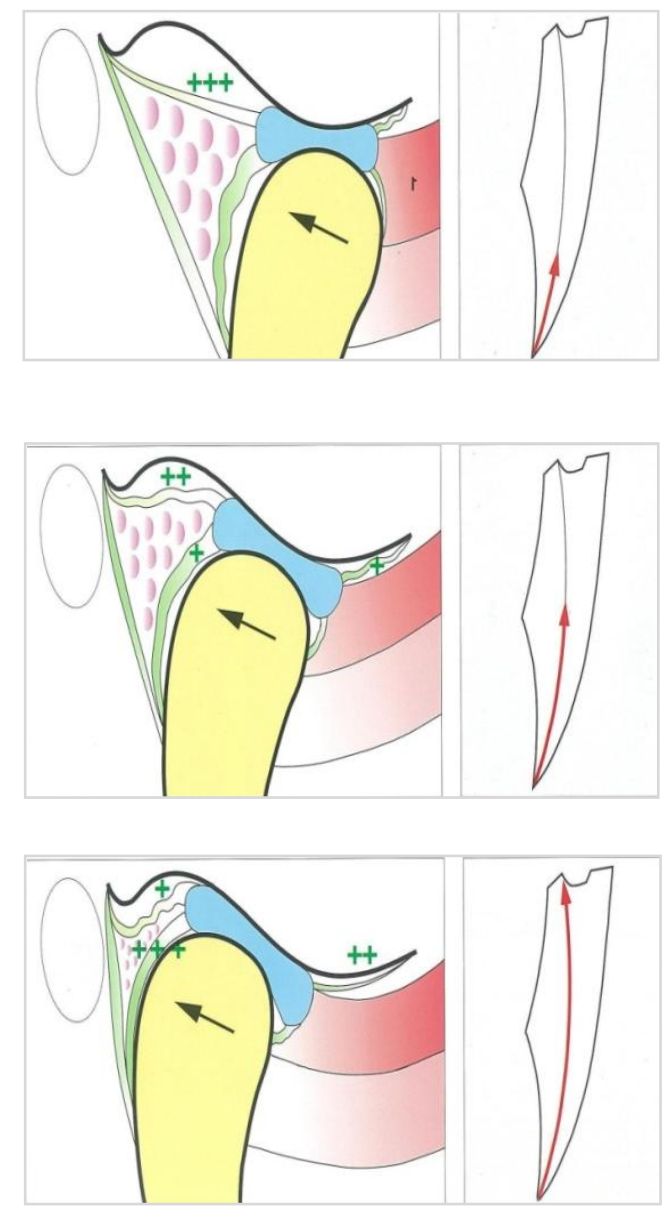

Abb. 3: „Bewegungsmuster Mundschluss 1. Initiale Phase: Schematische Darstellung der Belastungen während der initialen Mundschließbewegung. Die Dorsalbewegung des Kondylus wird durch das Caput superius des M. ptery. lateralis (1) gebremst. Der Diskus wird passiv nach dorsal geführt. Dies wird in dieser Phase durch die Spannung des Stratum superius möglich. Im Genu vasculosum entsteht ein physiologischer Überdruck (Abb. re.: entspr. PosseltDiagramm).

2. Intermediäre Phase: Das Caput superius stabilisiert weiterhin den Kondylus. Die Spannung im Stratum superius nimmt ab und der Diskus wird durch die Konvexität der Pars posterior weiterhin passiv nach dorsal geschoben (Abb. re.: entspr. Posselt-Diagramm).

3. Terminale Phase: Bei Mundschluss sind die elastischen Strukturen wieder entspannt. Das Stratum inferius kommt zunehmend auf Spannung und verhindert so bei übermäßiger Dorsalbewegung eine Verlagerung des Diskus nach anterior (Abb. re.: entspr. Posselt-Diagramm) “(Bumann und Lotzmann 2000 S. 47)

Das elastische Stratum superius ist in der initialen Schließungsphase für die Rückholbewegung des Diskus verantwortlich. Intermediär wird der Diskus - bedingt durch die Konvexität 
der Pars posterior - passiv mit nach dorsal geführt. Bei der terminalen Schlussrotation hält das straffe Stratum inferius den Diskus auf dem Kondylus. Bei physiologischen Bewegungen des Kondylus wird der Discus articularis durch den direkt am Diskus oder an der Kapsel ansetzenden M. pterygoideus lateralis und durch die elastischen Fasern der bilaminären Zone auf dem Kondylus so positioniert, dass Inkongruenzen ausgeglichen und Druckbelastungen ausgehend vom Kondylus auf die knöcherne Gelenkstruktur an der Schädelbasis vermieden werden (Bumann und Lotzmann 2000). 


\subsection{Pathophysiologie der Kraniomandibulären Dysfunktion (CMD)}

Eine große Vielfalt an Erkrankungen kann das muskuloskelettale System betreffen; die meisten können auch das Kiefergelenk mit einbeziehen. Einige dieser Erkrankungen sind relativ häufig und bekannt wie die Ostheoarthrose, die Ostheoarthritis und die rheumatische Arthritis, während andere wie infektiöse Erkrankungen und Stoffwechselerkrankungen seltener und für den Zahnarzt weniger relevant sind. Zu Beginn der für die CMD entwickelten Konzepte standen Gelenksprobleme im Blickpunkt (Carlson und Magnusson 1999). Dann wandte sich das Interesse der Muskulatur zu, der Hauptquelle von Schmerzen bei CMD (Carlson und Magnusson 1999). Während der 80er Jahre wurde allgemein angenommen, dass interne Gelenksveränderungen die Hauptfaktoren für die CMD seien. Heute ist allgemein anerkannt, dass eine Vielzahl verschiedener Störungen im Kiefergelenk und der Muskulatur gemeinsam oder getrennt zu einer CMD führt (Carlson und Magnusson 1999).

Die RDC/TMD von Dworkin (Dworkin und LeResche 1992) teilen die möglichen Pathophysiologien wie in der nachfolgenden Tabelle in drei Gruppen ein:

\begin{tabular}{|l}
\hline I. Muskelerkrankungen \\
\hline I.a. Myofaszialer Schmerz \\
I.b. Myofaszialer Schmerz mit eingeschränkter Mundöffnung
\end{tabular}

\section{Verlagerung des Discus articularis}

II.a. Verlagerung des Discus articularis mit Reposition

II.b. Verlagerung des Discus articularis ohne Reposition mit eingeschränkter Kieferöffnung

II.c. Verlagerung des Discus articularis ohne Reposition ohne eingeschränkte Kieferöffnung

\section{Arthralgie, Arthritis, Arthrose des Kiefergelenks}

III.a. Arthralgie

III.b. Arthritis

III.c. Arthrose

Tab.1: Einteilung der Pathophysiologien des Kiefergelenks nach Dworkin (Dworkin und LeResche 1992). 
Eine weitere mögliche Einteilung ist der Dysfunktionsindex nach Helkimo (1974), der vier Kriterien berücksichtigt:

\section{a) Unterkieferbeweglichkeit}

Eingeschränkte Unterkieferbeweglichkeit

Eingeschränkte Lateralbewegungen

Eingeschränkte Protusion

b) Gelenkfunktion

Kiefergelenksgeräusche (Reiben und Knacken)

Deviation bei Mundöffnung

c) Palpation der Muskulatur

Anzahl der Stellen der Muskulatur mit Palpationsempfindlichkeit

d) Palpation des Kiefergelenks

Schmerz bei Palpation von lateral

Schmerz bei Palpation von dorsal

Durch Bewertung der Teilindices a-d zusammen wird der Dysfunktionsindex definiert:

D0 keine klinische Dysfunktion

D1 leichte klinische Dysfunktion

D2 moderate klinische Dysfunktion

D3 schwere klinische Dysfunktion

Tab.2: Dysfunktionsindex nach Helkimo (Helkimo 1974).

Die weiteren Ausführungen sollen sich vor allen Dingen an die Einteilungsvorschläge der RDC/TMD halten, da diese international gebräuchlich sind und auch in dieser Arbeit eine übergeordnete Rolle spielen. Wichtig ist dabei allerdings, dass es neben der RDC/TMD auch weitere klinische Bewertungskriterien für die CMD gibt, wie sie hier mit dem Dysfunktionsindex von Helkimo (1974) kurz veranschaulicht werden sollen. 


\subsubsection{Myogene Dysfunktion}

\subsubsection{Pathophysiologie der myogenen Dysfunktion}

Geht man davon aus, dass das stomatognathe System über einen Regelkreis untereinander verbunden ist, das heißt, dass es Propriorezeptoren, Parodont, Muskelspindeln etc. gibt, die über Nerven, Leitungsbahnen zum Pons, Mesenzephalon, Thalamus und Gyrus postcentralis Informationen schicken, welche dann nach Verarbeitung über denselben Weg zurück eine entsprechende Muskelreaktion auslösen, so führen Störungen auch immer reflektorisch zu einer Muskelreaktion.

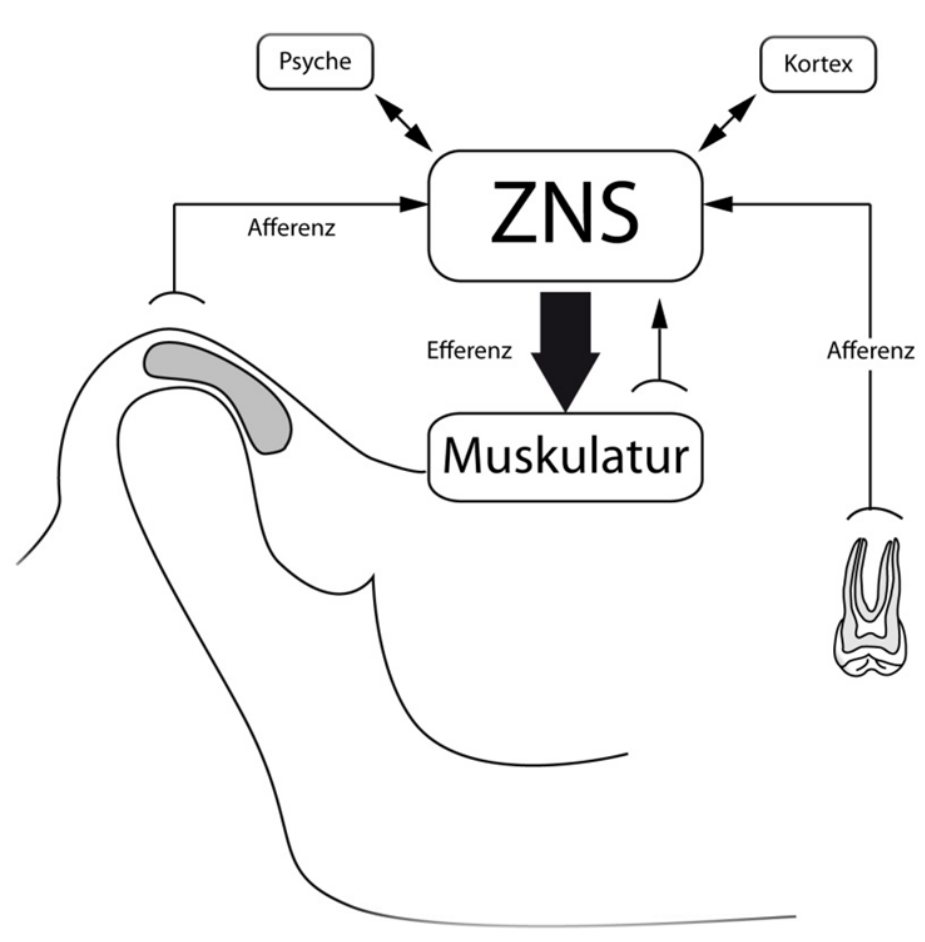

Abb.4: Physiologische myogene Funktion (Meyer et al. 2003 S. 19)

Während z.B. Fremdkörper als temporäre Störfaktoren muskulär völlig problemlos verkraftet werden, können langfristig auftretende Reizfaktoren zu einer Überlastung der Kaumuskulatur führen. So stellen langfristige Störfaktoren im Modell des Regelkreises Trigger dar und lösen reflektorisch Parafunktionen aus.

Dabei muss allerdings klar gestellt werden, dass auch die myogenen Kiefergelenkserkrankungen und Schmerzen multikausal bedingt sind (Clark 2008) und daher genauso wie die Diskopathien und die arthrogenen Kiefergelenkserkrankungen, lokal oder zentral ausgelöst, zu einer Hyperaktivität und somit zu einer Überlastung der Kaumuskulatur führen können (Cairns et al. 1998; Sunakawa et al. 1999). Dies zeigt aber auch, dass therapeutische 
Konzepte, welche auf die Muskulatur abzielen, bei jeder Art von Kiefergelenkserkrankungen anders als bei einer muskulären Genese zu einer Linderung des Schmerzbildes führen werden.

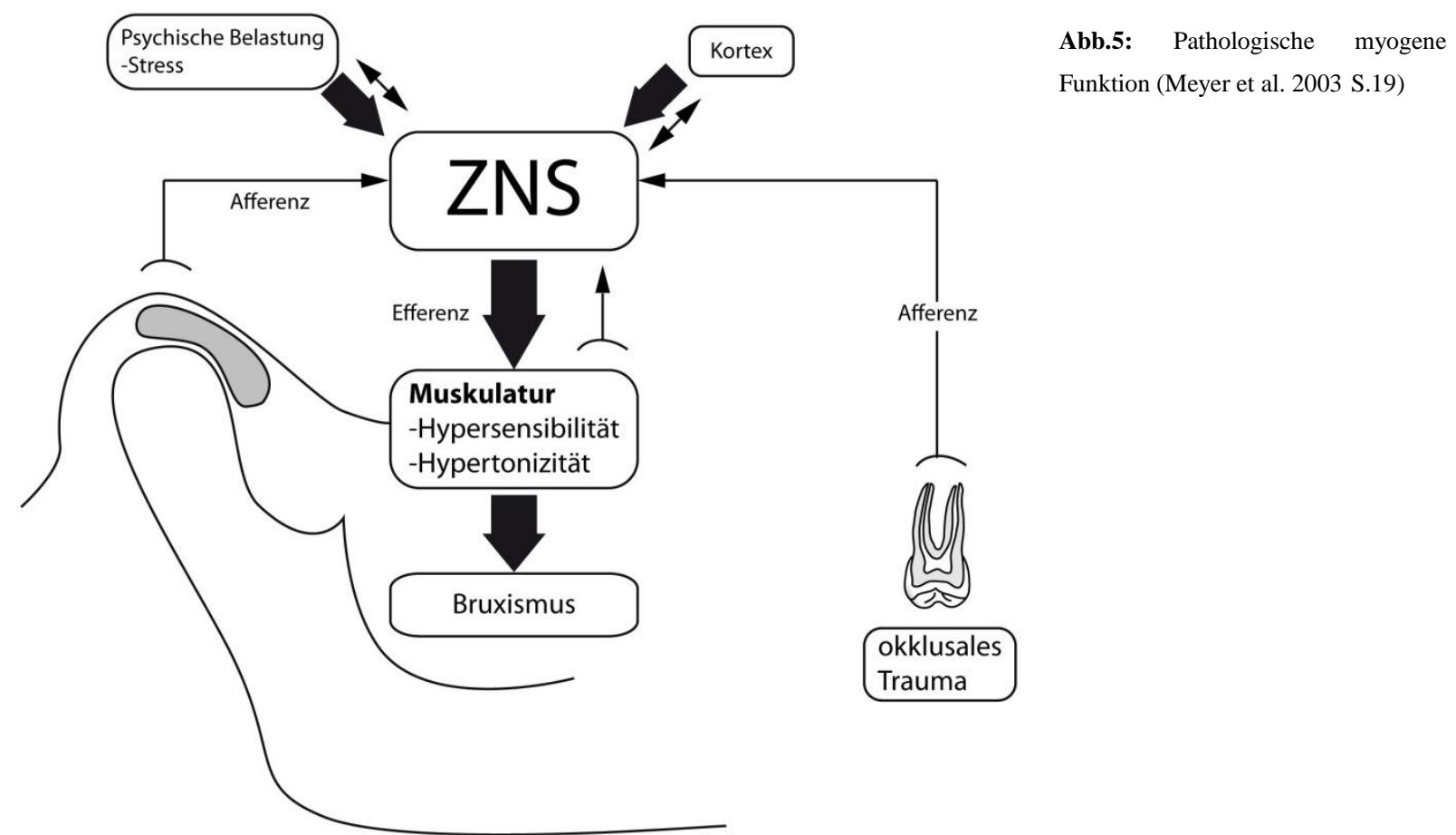

\subsubsection{Symptomatik der myogenen Dysfunktion}

Bei der Überbelastung der Muskulatur wird Adenosintriphosphat (ATP) verbraucht, was zu einer verstärkten Creatininphosphatspaltung führt, verbunden mit Sauerstoffmangel und Anreicherung von Milchsäure bzw. Histamin im Muskel. Die verminderte Sauerstoffzufuhr und der hohe Lactatspiegel können zu ischämischen Zuständen im Muskel und in dessen Folge zu Muskelhartspann, Verspannungen und Muskelschmerzen führen. Bleibt der Spannungszustand bestehen, können Myogelosen entstehen, stecknadelkopfgroße, spindelige, knotige, druckschmerzhafte Verhärtungen, die als Folge kolloidchemischer Veränderungen gewertet werden. 


\subsubsection{Therapie der myogenen Dysfunktion}

Ziel der Therapie muss es sein, das Reflexgeschehen zu unterbrechen und so nicht nur die Normalfunktion wieder herzustellen, sondern auch die Schmerz auslösenden Belastungszustände der myogenen Dysfunktion zu beseitigen. Dabei haben sich zwei Therapieprinzipien bewährt:

Zum einen myofunktionelle Übungen, die Elemente der Verhaltenstherapie als auch der physikalischen Medizin enthalten. Ziel dieser Übungen ist es, durch gezielte Selbstmassage der Kaumuskulatur den Muskelhartspann zu lösen und die schmerzhaften Myogelosen auszumassieren. Danach wird über Bewegungsübungen eine auf die Mittellinie des Patienten ausgerichtete Öffnungs- und Schließbewegung trainiert. Zusätzlich kann auch mit professioneller Physiotherapie gearbeitet werden.

Zum anderen können insbesondere bei Verdacht auf eine okklusale Genese temporäre Aufbissschienen angefertigt werden. Dabei kommt es durch die erhöhte Vertikaldistanz zu einer Vordehnung der Kaumuskulatur und somit zu einer Entspannung. Außerdem werden durch die in Zentrik angefertigten Schienen die durch Fehlokklusion ausgelösten pathologischen Reflexmechanismen aufgehoben (Schwenzer und Ehrenfeld 2000). Dabei liegt die Schwierigkeit der Behandlung darin, deren Komplexizität den Bedürfnissen des Patienten anzupassen (Fricton 2007).

\subsubsection{Formen und Einteilung von Diskopathien}

\subsubsection{Pathophysiologie der Diskopathien}

Diskopathien sind durch eine pathologische Diskus-Kondylus-Fossa-Relation charakterisiert. Fast immer ist der Diskus nach anterior oder anteriomedial verlagert. Es werden jedoch auch Einzelfälle von lateral oder posterior verlagerten Diski beschrieben (Blankestijn und Boering 1985; Gallagher 1986; Vasconcelos et al. 2010). Dabei ist für die Entstehung einer Diskusverlagerung die Überdehnung oder im Extremfall das Reißen der posterioren Bänder Voraussetzung. Diese entsteht durch eine dorsokraniale Kondylenverlagerung, welche Folge einer reduzierten dentalen Abstützung des Unterkiefers im Seitenzahnbereich als auch eines Traumas mit dorsaler Krafteinwirkung auf den Kondylus sein kann (Schwenzer und Ehrenfeld 2000). 


\subsection{Morphologische Pathologien des Discus articularis:}

\subsection{Morphologie der Pars posterior des Discus articularis}

a.) physiologische Pars posterior

Die bikonvexe Form ist für die physiologische Pars posterior charakteristisch. Der Diskus kann sich nur erschwert nach anterior verlagern und wird somit auf dem Kondylus stabilisiert, weil die Pars posterior dicker ist als der Abstand zwischen der ventrokranialen Kontur des Kondylus und der Protuberantia

b.) biplanare Pars posterior

Der Verlauf der kraniale und kaudale Fläche der Pars posterior ist annähernd parallel. Es sollten im MRT immer Aufnahmen bei geschlossenem und geöffnetem Mund befundet werden, da sich die Form der Pars posterior bei diesen unterschiedlich darstellen kann.

c.) keilförmig abgeflachte Pars posterior

Bei anhaltenden kraniale oder dorsokranialen Belastungen, kann diese Formveränderung auftreten (Bumann und Lotzmann 2000).

\subsection{Adhäsion des Discus articularis :}

a.) Diskusadhäsion ohne Diskusverlagerung

Bei habitueller Okklusion zeigt das MRT eine geringgradige Tendenz zur anterioren Diskusverlagerung. der Kondylus steht Bei maximaler Mundöffnung fast auf dem Zenit der Eminentia und die Lage des Diskus ist dabei unverändert.

b.) Diskusadhäsion mit Diskusverlagerung

Bei habitueller Okklusion zeigt das MRT eine eindeutige anteriore Diskusverlagerung. Beurteilt man das MRT bei maximaler Mundöffnung, so ist der Diskus reponiert. Gleichzeitig ist die Translation aber eingeschränkt.

c.) Diskusadhäsion mit Kondylushypermobilität

Bei habitueller Okklusion ist die Diskus-Kondylus-Relation als intakt zu betrachten. Bei maximaler Mundöffnung zeigt der Diskus nur eine minimale Translation. Anzunehmen ist, dass der Kondylus durch seine Hypermobilität den Diskus nach ventral verlassen hat (Bumann und Lotzmann 2000) .

\subsection{Hypermobilität des Discus articularis :}

Die Diskushypermobilität kann ein initiales Stadium einer eindeutigen anterioren Diskusverlagerung darstellen. Dadas Erscheinungsbild im MRT sehr variieren kann, ist sie meist nicht von einer partiellen Diskusverlagerung zu unterscheiden (Bumann und Lotzmann 2000).

\subsection{Durch Verlagerungen bedingte Pathologien des Discus articularis:}

\subsection{Partielle Verlagerung des Discus articularis nach anterior}

Die Bezeichnung partielle oder totale Diskusverlagerung ist unabhängig von der Reposition des Diskus. die Verlagerung des Diskus in einer Schicht reicht für einen Befund aus. Aber sie ist immer ein Summationsbefund der Diskuspositionen in medialen, zentralen und lateralen Gelenkanteilen. Letztendlich führt ist eine Überdehnung der dorsolateralen Anteile des Stratum inferius zur partiel- 
len Diskusverlagerung. Hierfür ist ein dorsolateraler oder dorsokraniolateraler Belastungsvektor erforderlich.

a.) ohne Reposition

Bei habitueller Okklusion ist der Diskus partiell nach anterior verlagert und leicht deformiert. Weiterhin sind oft degenerative Veränderungen am Kondylus zu finden. Kondylus und Diskus können bei maximaler Mundöffnung eine weite Bewegung nach ventral ausführen. Zu einer Reposition kommt es jedoch nicht.

b.) partielle Reposition

Bei habitueller Okklusion ist der Diskus partiell nach anterior verlagert. Die Pars posterior ist nicht deformiert. Bei maximaler Mundöffnung haben Diskus und Kondylus eine deutliche Translation nach ventral vollzogen. Der Zenit des Kondylus steht als Zeichen einer unvollständigen Reposition auf der Pars posterior und nicht auf der Pars intermedia.

\section{c.) totale Reposition}

Bei habitueller Okklusion ist der Diskus partiell nach anterior verlagert, und die Pars posterior ist abgeflacht (Abb.4 Punkt 1). Bei maximaler Mundöffnung hat der Kondylus eine deutliche Translation nach ventral ausgeführt. Da der Diskus eine Relativbewegung nach dorsal vollzogen hat, kommt es zur vollständigen Reposition des Diskus (Abb.4 Punkt 4) (Bumann und Lotzmann 2000).

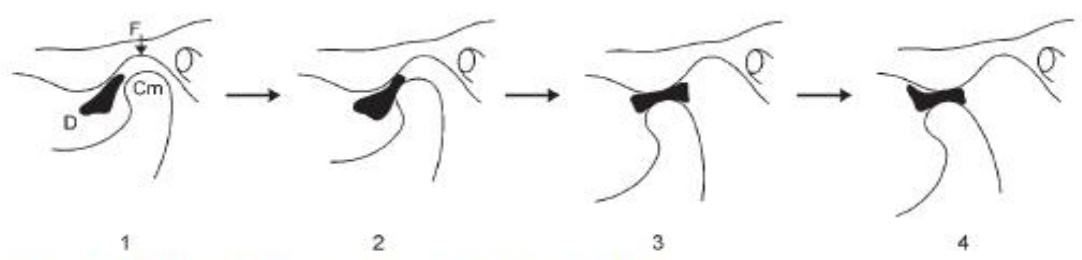

Abb.6: Diskusverlagerung mit totaler Reposition. $\mathrm{F}=$ Fossa articularis, $\mathrm{Cm}=$ Collum mandibulae, $\mathrm{D}=$ Discus articularis (Krestan et al. 2001 S. 744)

\subsection{Totale Verlagerung des Discus articularis nach anterior}

Die totale Verlagerung des Discus articularis nach anterior ist immer ein Summationsbefund der Diskuspositionen in medialen, zentralen und lateralen Gelenkanteilen, wobei die Verlagerung des Diskus in allen drei Schichten für einen Befund nötig ist. Die Bezeichnung partielle oder totale Diskusverlagerung ist unabhängig von der Reposition des Diskus. Die totale Diskusverlagerung ist gleichzusetzen mit einer Überdehnung des gesamten Stratum inferius, wofür ein dorsaler oder dorsokranialer Belastungsvektor erforderlich ist.

a.) ohne Reposition

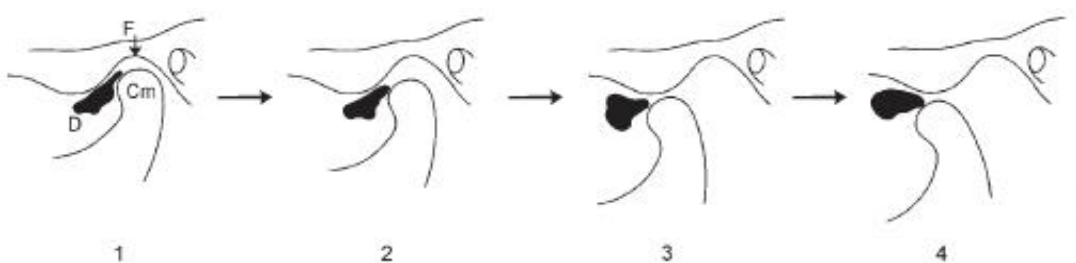

Abb.7: Diskusverlagerung ohne Reposition. $\mathrm{F}=$ Fossa articularis, $\mathrm{Cm}=$ Collum mandibulae, $\mathrm{D}=$ Discus articularis (Krestan et al. 2001 S. 746) 
Bei habitueller Okklusion ist der Diskus nach anterior verlagert und leicht deformiert (Abb.5 Punkt 1). Zudem bestehen oft degenerative Veränderungen am Kondylus. Bei maximaler Mundöffnung können Kondylus und Diskus eine weite Bewegung nach ventral ausführen, wobei es jedoch nicht zur Reposition kommt (Abb.5 Punkt 4).

b.) partielle Reposition

Bei habitueller Okklusion ist der Diskus nach anterior verlagert. Die Pars posterior ist nicht deformiert. Bei maximaler Mundöffnung haben Diskus und Kondylus eine deutliche Translation nach ventral vollzogen. Der Zenit des Kondylus steht als Zeichen einer unvollständigen Reposition auf der Pars posterior und nicht auf der Pars intermedia.

a.) totale Reposition

Bei habitueller Okklusion ist der Diskus nach anterior verlagert und die Pars posterior ist abgeflacht. Bei maximaler Mundöffnung hat der Kondylus eine deutliche Translation nach ventral ausgeführt. Da der Diskus eine Relativbewegung nach dorsal vollzogen hat, kommt es zur vollständigen Reposition des Diskus (Bumann und Lotzmann 2000).

\subsection{Verlagerung des Discus articularis nach posterior}

Diese Art der Verlagerung entsteht oft nach Traumata und ist relativ selten. Dabei liegt der Kondylus bei geöffnetem und geschlossenem Mund ventral $\mathrm{zu}$ der Pars anterior des Discus articularis. Beim Mundschluss wird der Diskus im retrokondylären Raum komprimiert (Bumann und Lotzmann 2000).

\subsubsection{Symptomatik der Diskopathien}

Zunächst einmal muss man festhalten, dass Diskopathien auch symptomlos auftreten können und mit bis zu 22\% auch keine Seltenheit darstellen (Larheim et al. 2001). Also scheint es keine enge Korrelation mit strukturellen Variationen des Diskus-Kondylus-Komplexes und der klinischen Symptome zu geben.

Überdies variieren die mit Diskopathien einhergehenden Symptome sehr stark. Sie umfassen meist Schmerz, Palpationsempfindlichkeit der Gelenke und/oder der Muskulatur, Gelenkgeräusche und eingeschränkte Kieferbeweglichkeit. Dabei besteht in der Differntialdiagnostik das Problem, dass Kiefergelenksgeräusche am unzuverlässigsten von allen Symptomen sind (Carlson und Magnusson 1999; Kobs et al. 2005). 


\subsubsection{Therapie der Diskopathien}

Bei der Mehrzahl der Patienten sollte zunächst eine einfache Therapie mit Anweisungen, ggf. Medikation und physikalischer Therapie, oft kombiniert mit einer Aufbissschiene, versucht werden. Studien haben gezeigt, dass einige Diskopathien eher mit Schmerz und Dysfunktion korrelieren als andere (Larheim et al. 2001). Viele Personen, die im Röntgenbild oder MRT eine Diskusverlagerung zeigen, haben keine Symptome einer CMD. In der Allgemeinpraxis ist es daher nicht notwendig, hochtechnische Imaging-Techniken einzusetzen, um klinische Diagnosen einer Diskusverlagerung zu erstellen. Bei den meisten Patienten reicht ein einfacher Ansatz zur klinischen Diagnose und Therapie aus. Wenn nach einer solchen Behandlung die Symptome Schmerz und Dysfunktion immer noch persistieren, sollte der Patient zu einem Spezialisten überwiesen werden (Carlson und Magnusson 1999).

\subsubsection{Pathophysiologie des Kiefergelenks bei CMD}

\subsubsection{Bilaminäre Zone}

Hier dominieren histologisch Fibroblasten, dickwandige Arterien, dünnwandige Venen und zahlreiche vaskuläre Sinus (Wish-Baratz et al. 1993). Trotz des hohen Glykosaminoglykananteils der Matrix kommen physiologisch im Gelenk keine Chondrozyten vor. Eine Belastung der bilaminären Zone durch dorsokraniale oder dorsale Belastungsvektoren führt zu einer Stimulation der Kollagensynthese, was mit einer progressiven Adaption zu vergleichen ist, oder zu einer entzündlichen, schmerzhaften Veränderung, welche mit einer regressiven Adaption gleichzusetzten ist. Progressiv adaptiertes Bindegewebe wird durch Ablagerung polyanionischer Glykosaminoglykane gegen Belastung resistenter, es entsteht ein sog. „Pseudodiskus“ (Scapino 1983; Blaustein und Scapino 1986). Eine progressive Adaptation der bilaminären Zone ist nicht von der Diskusposition, sondern von der Richtung und vom Ausmaß der Gelenkbelastung abhängig. Fibrosierungen treten bei physiologischer Diskusposition sowie bei Diskusverlagerungen mit oder ohne Reposition auf. Sie sind therapeutisch von großer Bedeutung, da mehr als $90 \%$ der Gelenkschmerzen von der bilaminären Zone stammen (Bumann und Lotzmann 2000). 


\subsubsection{Discus articularis (Perforation)}

Initiale Phase der Osteoarthrose (s.u.). Auch hier kommt es durch Gelenksüberbelastung und/oder eingeschränkter Adaptionskapazität des Knorpels zur Abnutzung des Diskus bis hin zur vollständigen Perforation, teilweise mit Krepitus (Bumann und Lotzmann 2000).

\subsubsection{Erkrankungen des Kiefergelenks}

Zunächst einmal sind die Begriffe Osteoarthrose und Osteoarthritis voneinander abzugrenzen, da sie nicht die gleiche Ätiologie, Pathologie und Behandlung haben. Die Osteoarthose ist eine nicht entzündlich bedingte Verschleißerscheinung an Gelenken. Die Osteoarthritis ist eine entzündlich bedingte degenerative Erkrankung von Gelenken. Dabei muss man aber z.B. die rheumatische Arthritis, welche auch die Kiefergelenke befallen kann, von der durch eine Dysfunktion ausgelösten Osteoarthritis unterscheiden. Diese besitzt eine andere Pathophysiologie als die Rheumatische Arthritis, ist dabei zwar auch durch eine niedrige Anzahl entzündungskennzeichnender Botenstoffe zu diagnostizieren, aber nicht durch eine extrem hohe Anzahl charakterisiert. So kann durch eine Abnahme der adaptiven Fähigkeiten der am Kiefergelenk beteiligten Strukturen und/oder durch exzessive mechanische Belastungen, die die normalen adaptiven Fähigkeiten übersteigen, fehlerhafte Gewebebildungen induzieren. Hierbei wirken funktionelle Überbelastungen und eine dadurch erhöhte Gelenksfriktion als Synergisten zusammen (Tanaka et al. 2008). In den Chondrozyten des Kiefergelenks kommt es bei Zugbelastung zur vermehrten Expression von Matrix-Metalloproteinasen (MMP-13) und vaskulärem endothelialen Wachstumsfaktor (VEGF), während die Expression des Gewebeinhibitors für MMP-1 reduziert wird. Dieser Effekt ist bei hydrostatischem Druck genau umgekehrt (Wong et al. 2003). Hierdurch und durch die bei funktioneller Überlastung entstehende Hypoxie im Kiefergelenk kommt es über die VEGFs, welche die Produktion der MMP's und des Gewebeinhibitors TIMP-1 regulieren, zu einem Ungleichgewicht in der Herstellung der Komponenten der extrazellulären Matrix, Kollagen und Proteoglykane, so dass diese schneller abgebaut als neu hergestellt werden. Dieses Ungleichgewicht führt dann zu einer Zerstörung des Gelenkknorpels (Pufe et al. 2004). Die Überlastung hat außerdem zur Folge, dass die regelgerechte Versorgung der Kiefergelenke mit Synovialflüssigkeit nicht mehr gewährleistet ist. Dies ist ein Resultat des Abbaus der Hyaloronsäure durch freie Radikale. Die Produktion der Hyaluronsäure wird von verschiedenen Entzündungsmediatoren bewirkt. Dabei spielen der Tumor-Nekrose-Faktor $\alpha$ (TNF $\alpha$ ) und Interleukin-1 und -6 eine entscheidende Rolle für die degenerativen Prozesse im Zuge der Ostheoarthritis. Sie sind vor allen Dingen für die Beschleunigung und Progression der pathologischen Prozesse verant- 
wortlich, weil sie die Knochenresorption über die Differenzierung und Aktivierung von Osteoklasten steuern und kontrollieren. Somit unterhalten sie die degenerativen Prozesse im Kiefergelenk und fördern den Abbau der am Kiefergelenk beteiligten Strukturen, bis eine physiologische Funktion in diesem Stadium dann bereits nicht mehr möglich ist (Tanaka et al. 2008).

Zudem existieren auch noch nicht-arthritisch bedingte Kiefergelenksschmerzen, sog. Arthralgien. Diese können traumatischer oder infektiöser Genese sein. Manch eine systemische Erkrankung kann auch mit Kiefergelenksschmerzen einhergehen. Hier wären die ankylosierende Spondylitis, Gicht und Akromegalie zu nennen. Noch seltener sind Tumore im Bereich des Kiefergelenks. Die Prävalenz dieser Erkrankungen ist sehr gering, muss aber bei einer Untersuchung immer als Möglichkeit in Betracht gezogen bzw. in der Anamnese erfragt werden (Carlson und Magnusson 1999). 


\subsection{Zahnmedizinische Diagnostik}

Neben der rein manuellen Untersuchung des Patienten bieten sich dem Zahnarzt vermehrt und dazu noch hoch technisierte Methoden an, um das Kiefergelenk zu untersuchen. Dabei ist die manuelle Untersuchung als erstes Screening des Patienten immer noch aussagekräftig und für den alltäglichen Gebrauch in der Praxis völlig ausreichend. Bei Patienten mit schwerwiegenderen Pathologien ist dann eine bildgebende Untersuchung unerlässlich, um eine gesicherte Diagnose stellen und eine gezielte Therapie einleiten zu können. Beim heutigen Stand der Technik ist es also möglich, jedem Patienten die für ihn aussagekräftigste Untersuchung zukommen zu lassen (Brooks et al. 1997).

\subsubsection{Research Diagnostic Criteria for Temporomandibular Disorders (RDC/TMD)}

1992 wurden die RDC/TMD auf der Basis von Expertenmeinungen und den vorhandenen empirischen Daten entwickelt. Ihre Intention war es, ein Diagnose- und Klassifikationssystem anzubieten, welches dafür sorgen sollte, zukünftige Forschungen zu unterstützen. Außerdem sollte es so angelegt werden, dass die Untersuchungen und deren Ergebnisse in der klinischen Praxis erleichtert werden, um den CMD-Patienten besser helfen zu können (Dworkin 2010). Die RDC/TMD orientiert sich dabei an den in Tab.1 gezeigten Untergruppen der CMD, welche indessen keinen Anspruch auf Vollständigkeit erheben. Dabei teilen sie sich in ihren Bewertungskriterien in zwei Achsen auf:

Achse I dient der Feststellung einer Diagnose zum einen aus der klinischen Geschichte des Patienten heraus und zum anderen aus der manuellen Untersuchung des Zahnarztes. Dabei werden dem Behandler sowohl die anamnestischen Fragen als auch die manuelle Untersuchung vorgegeben, um im Endeffekt evidenzbasiert mit den Ergebnissen arbeiten zu können.

Achse II dient dazu, den Schmerzlevel, die Unterkieferfunktion sowie die sozialen und psychosozialen Faktoren zu erfassen. Auch hier werden für den Behandler sowohl Fragen und Untersuchungen als auch Einteilungen für Schmerzparameter vorgegeben, damit auf dieser Grundlage evidenzbasiertes Arbeiten möglich wird (John et al. 2006).

Der Konsens aus beiden Achsen sollte es dem Behandler ermöglichen, eine eindeutige Diagnose zu stellen, aus der er dann eine geeignete Therapie ableiten kann (Dworkin 2010). 
Demzufolge wurde auch bei der Neufassung des klinischen Funktionsstatus der Arbeitsgemeinschaft für Funktionslehre der DGZMK eine Einbeziehung evidenzbasierter Erkenntnisse unter Zuhilfenahme der RDC/TMD gefordert (Ottl et al. 2005). Überdies zeigten die Übersetzung der RDC/TMD in mehr als 20 Sprachen und dass sie bis 2005 über 600 mal zitiert wurden, dass sie die an sich selbst gestellten Kriterien eines evidenzbasierten Arbeitens und einer klinischen Anwendbarkeit zum Wohle des CMD-Patienten erfüllen. Allerdings sind über die Jahre auch Kritikpunkte aufgetreten, welche aber nach deren Aufarbeitung eher zu einer Verbesserung der RDC/TMD führten und deren Nutzen letztlich nicht in Frage stellen sollten (Dworkin 2010).

\subsubsection{Bildgebende Diagnostik}

In der bildgebenden Diagnostik hat sich auf Grund des technischen Fortschritts in den letzten Jahren einiges verändert. So ist heute ein Wandel von der konventionellen Tomographie und der invasiven Arthrographie des Kiefergelenks zur Computertomographie (CT) und Magnetresonanztomographie (MRT) eingetreten. Diese gelten nunmehr je nach Fragestellung als Standarduntersuchung (Jäger et al. 2001).

Dabei können sie nach einer klinischen Untersuchung und bei Verdacht auf eine Strukturveränderung des Kiefergelenks zur Diagnosesicherung eingesetzt werden. Jedoch treffen sie in der Regel keine Aussage über die Kiefergelenksfunktion, da sie meist statisch sind. Ändern könnte dies die real-time-MRT (Zhang et al. 2011) oder auch die hochauflösende Sonographie, da sie keine Einzelbilder mehr liefern, sondern Videosequenzen der Kiefergelenke während ihrer Funktion (Emshoff et al. 2002 b). Eine Indikation zur Untersuchung der Kiefergelenksstrukturen liegt nach einer klinischen Untersuchung dann vor, wenn der Verdacht auf eine primäre Gelenkserkrankung, eine sekundäre morphologische Erkrankung oder eine pathologische Kondylus-Diskus-Lagebeziehung besteht.

\subsubsection{Röntgenologische Untersuchung}

Knöcherne Anteile des Kiefergelenks können entweder mit dem MRT oder mit röntgenologischen Verfahren dargestellt werden. Dazu gehören das Orthopantomogramm (OPG), welches mittels spezieller Zusatzprogramme für Gelenkaufnehmen ausgerüstet werden kann, ferner 
das Fernröntgenseitenbild, die axiale Schädelaufnahme nach Hirtz, die transkraniale Schädelaufnahme nach Schüller, die okzipitofrontale Schädelaufnahme nach Clementschitsch und die CT. Aber bis auf das OPG und die CT haben die hier aufgeführten Verfahren nur eine geringe Aussagekraft bei der Diagnosesicherung einer CMD. Dies ist den außer bei der Arthrographie enstehenden Überlagerungen geschuldet. Diese entstehen, wenn sich knöcherne Anteile überlagern und so aussagekräftige Befunde durch die röntgenologischen Schatten erschwert werden (Brooks et al. 1997).

\subsubsection{Computertomographische Untersuchung}

Indikationen sind sowohl die Frakturdiagnostik als auch die Erkennung von arthritischen und neoplastischen Veränderungen sowie von Ankylosen (Brooks et al. 1997). Dabei lassen sich die Kiefergelenksstrukturen am besten mit einem Spiralmodus darstellen. Bei diesem Mehrzeilen-CT lassen sich im Nachhinein alle Bildgebungsprojektionen frei rekonstruieren, so dass unter anderem die bekannten koronaren und sagittalen Projektionen möglich sind. Diese erfolgen in Rückenlage bei habitueller Interkuspidation. Die Projektionen können dann multiplanar rekonstruiert werden, so dass man Knochenbrüche des Kondylus oder etwaige andere Frakturen und eventuelle arthritische Veränderungen identifizieren und darstellen kann. Eine Alternative zum konventionellen CT bietet die Cone Beam Computed Tomography (CBCT). Sie verkürzt die Untersuchungszeit, verringert die Strahlendosis und auch das Investitionsvolumen ist geringer. Dabei lässt sich die Bildqualität mit der der konventionellen CT durchaus vergleichen (Barghan et al. 2010).

\subsubsection{Magnetresonanztomographische Untersuchung}

Abb.5 zeigt eine Sequenz von sagittalen Schnitten durch ein Kiefergelenk. Dabei sind deutlich größere anatomische Strukturen wie der Kondylus (a), der Porus acusticus externus (b) oder die Fossa und die Eminentia mandibularis (c) genauso zu erkennen wie auch Teile der Kaumuskulatur. Aber auch feine Strukturen wie der Discus articularis (d) und die bilaminäre Zone mit ihren einzelnen Kompartimenten treten deutlich hervor. Ziel einer MRTUntersuchung ist somit, Pathologien innerhalb dieser Strukturen zu erkennen und Aufschlüsse für die Diagnosefindung zu liefern. 

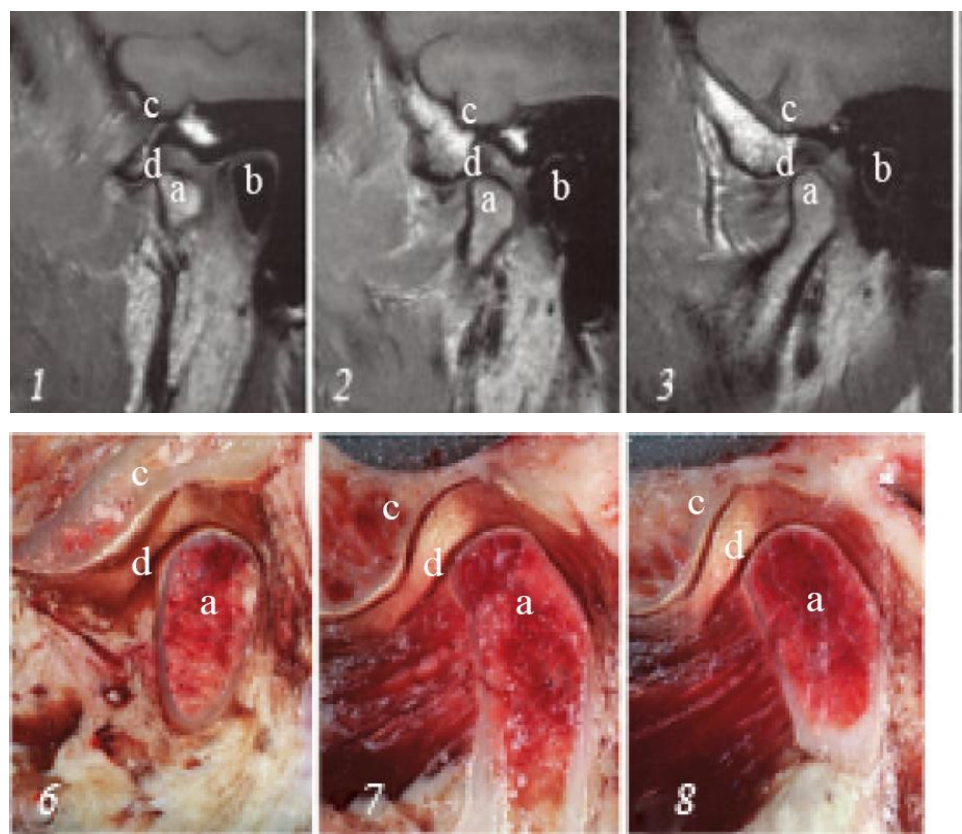

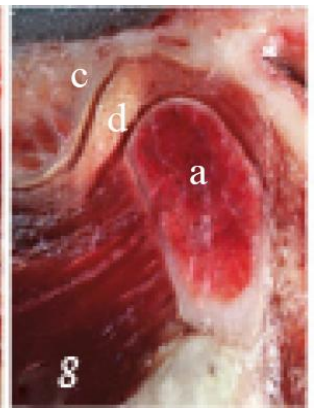

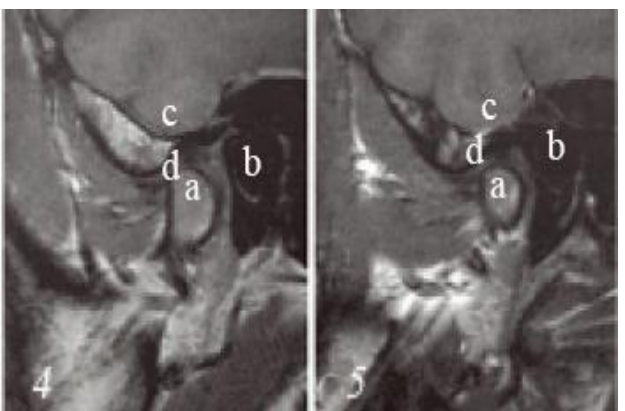

Abb. 8: Normale Anatomie-MRT (1-5) in symptomfreien Freiwilligen bei geschlossenem Mund und Autopsieproben (6-7). 1. Laterales Bild. 2. Laterozentrales Bild. 3. Zentrales Bild 4. Zentromediales Bild. 5.Mediales Bild 6. Laterales Bild. 7. Zentrales Bild. 8. Mediales Bild. Normale Diskusform und Position als auch unauffällige Knochen (modifiziert Larheim 2005 S. 8)

Auch bei der MRT wird der Patient in Rückenlage untersucht. Meist werden MRT-Geräte von mindestens 1 Tesla (T) oder mehr und Kiefergelenksspulen verwendet. Durch die Kiefergelenksspulen kann im Bereich der Kiefergelenke eine bessere Auflösung durch ein gutes Signal-Rausch-Verhältnis erzielt werden. Dabei ist es aber notwendig, dass die Spulen möglichst genau über dem Kiefergelenk platziert werden. Hier kann als Orientierung der äußere Gehörgang dienen, da die Spulen immer vor diesem platziert werden sollten.

Ein erstes Bild muss immer mit einem großen "Field of view" (FOV) in einer T1-Gewichtung gemacht werden. Auf ihm sollten beide Kiefergelenke zu sehen sein, da es als Orientierung und zur Planung der folgenden Aufnahmen gebraucht wird (sog. Localizer). Diese erfolgen nun meist seitengetrennt nacheinander. Dabei lässt sich auch die individuelle Kondylenbahnachse jedes Patienten und jeder Kiefergelenksseite in Relation zur Frontalebene festlegen. Das Kiefergelenk kann nun in kontinuierlichen Schichten untersucht werden. Die Wahl der Dicke der Schichten hängt dabei davon ab, welche Qualität man bei welcher Messzeit erreichen möchte. So ist eine Schichtdicke von $3 \mathrm{~mm}$ üblich. Will man jedoch z.B. bei einer Diskopathie eine höhere Detailauflösung erreichen und verkleinert dazu die Schichtdicke auf $2 \mathrm{~mm}$, so muss man die Messzeit verlängern, um ein adäquates Signal-Rausch-Verhältnis zu gewährleisten. Die Untersuchungsebenen lassen sich in diesem Fall parallel zur Kondylenachse und/oder senkrecht zu dieser wählen (Jäger et al. 2001).

Weiterhin lassen sich durch unterschiedlich gewichtete Sequenzen bestimmte Areale besser auflösen. Soll die Auflösung noch erhöht werden, kann auch bei Entzündungen mit einem Kontrastmittel gearbeitet werden, welches intravenös appliziert wird (Gadolinium-DTPA). 
Anatomische Detailauflösungen erfolgen meist mit protonengewichteten Sequenzen, wie z.B. einer 2D-FLASH-Sequenz (fast low angle shot) in parasagittaler Schichtführung. Der Diskus, der Kondylus, die bilaminäre Zone sowie das diskotemporale und diskokondyläre Gelenkkompartiment lassen sich auf diese Art und Weise gut abgrenzen (Jäger et al. 2001). Diskopathien sollten nicht nur in habitueller Interkuspidation, sondern auch mindestens in maximalen bzw. in unterschiedlich großen Winkeln bei der Mundöffnung mit Hilfe von Mundspreizern untersucht werden. Hierbei gab es bereits Versuche, die Serien von einzelnen gewonnenen Bildern durch deren Verknüpfung mittels eines Computerprogramms zusammenzufügen und damit eine pseudo-dynamische MRT-Aufnahmetechnik (CINE-Mode) zu entwickeln. Diese konnte aber bis heute noch keine Vorteile gegenüber der statischen MRTAufnahme liefern, obwohl eine dynamische Untersuchung der Kiefergelenksfunktion von den Behandlern durchaus als wünschenswert betrachtet wird (Behr et al. 1996). Die vorliegende Arbeit untersucht, ob die hier vorgestellte real-time-MRT die Schwächen der pseudodynamischen MRT überwinden kann (Zhang et al. 2011). Der Nutzen liegt darin, dass aus diesen Untersuchungsmethoden in Bezug auf die Diskus- und Kondylusmobilität mehr Informationen gewonnen werden können als aus statischen MRT-Aufnahmen (Eberhard et al. 2000). Besitzt der Patient zur Therapie bereits eine Aufbissschiene, so sind Aufnahmen mit dieser auch sinnvoll (Jäger et al. 2001). 


\subsection{Fragestellung}

Die Research Diagnostic Criteria for Temporomandibular Disorders (RDC/TMD) sind ein seit Jahren etabliertes und anerkanntes Verfahren zur Stellung von Diagnosen und deren evidenzbasierter Kontrolle. Dabei unterliegen sie seither der stetigen Verbesserung durch weltweiten wissenschaftlichen Austausch über gesammelte Erfahrungen und daraus entstandene Verbesserungsvorschläge (Dworkin 2010).

Nun hat sich in den letzten Jahren die Magnetresonanztomographie ebenfalls weiterentwickelt und ist bis zum heutigen Tage der „Goldstandard“ in der bildgebenden Diagnostik für CMD (Krestan et al. 2001). Dabei ist die real-time-MRT für das Kiefergelenk (Zhang et al. 2011) eine Weiterentwicklung der MRT. Jedoch muss sie noch den Beweis antreten, ebenso als „Goldstandard“ für die Untersuchung von an einer CMD leidenden Patienten zu gelten, wenn ihn nicht sogar noch zu übertreffen.

Daher legt diese Studie ihr Augenmerk darauf, ob und wie sich die Ergebnisse der RDC/TMD mit denen der real-time-MRT vergleichen lassen und ob letztere einen Mehrwert an Informationen für einen Behandler bieten können. 


\section{Material und Methoden}

Die Studie ist in Zusammenarbeit des Zentrums für Zahnmedizin der Medizinischen Fakultät der Universität Göttingen (Abteilung Prothetik; komm. Leiter PD. Dr. N. Gersdorff) mit der Biomedizinischen NMR-Forschungs GmbH am Max-Planck-Institut für biophysikalische Chemie (Direktor Prof. Dr. Jens Frahm) entstanden.

\subsection{Patientenkollektiv}

Bei dem Patientenkollektiv handelte es sich um Zahnmedizinstudenten/-innen der Universität Göttingen. Dabei wurden 30 Probanden, davon acht männlichen und 22 weiblichen Geschlechts im Alter zwischen 20 und 31 Jahren, mit einem Durchschnittsalter von 24,5 Jahren untersucht. Die Vorgeschichte ergab, dass die Probanden noch keine Untersuchung in Bezug auf eine CMD erhalten haben. Zunächst wurden alle Probanden auf der Grundlage der zu den RDC/TMD ergangenen Richtlinien untersucht und dann mit Hilfe des real-time-MRT gescannt. Vorher wurden alle Probanden über die MRT-Untersuchung und deren Risiken aufgeklärt. Alle erklärten sich einverstanden mit der Untersuchung. Des Weiteren lag auch ein positives Votum der Ethikkommission der Universitätsmedizin Göttingen vor. 


\subsection{Erhebung der Daten für die RDC/TMD}

Im Rahmen der Erhebung der Daten für die RDC/TMD wurden Fragebögen entwickelt (Dworkin und LeResche 1992), die sowohl anamnestische Fragen als auch solche zur Einflussnahme der CMD auf das tägliche Leben enthalten. Daneben wurden in den RDC/TMD stets auch epidemiologische Fragen gestellt, die aber für die vorliegende Arbeit keine Relevanz besaßen und somit vernachlässigt werden konnten. Ferner wurden die klinischen Untersuchungen streng nach Anleitung der RDC/TMD durchgeführt und lassen sich in zwei Achsen aufteilen:

- Achse I beschäftigt sich vor allen Dingen mit somatischen Beschwerden.

- Achse II beschäftigt sich mit psychosomatischen Beschwerden (Da diese für den Inhalt und das Ergebnis dieser Arbeit nicht von Belang und die Freiwilligen schmerzfrei waren, blieb Achse II unberücksichtigt).

Die in Tab. 2 - 4 dargestellten Fragen wurden den RDC/TMD entnommen (Dworkin und LeResche 1992). Die 30 Probanden wurden entlang der entwickelten Fragebögen vor der MRT-Untersuchung befragt und untersucht. Hierbei sei noch erwähnt, dass die Antworten durch Zahlen codiert sind, welche später dazu dienen, in einem vereinfachten Verfahren eine schnelle und sichere Diagnose anhand der RDC/TMD stellen zu können.

\subsubsection{Anamnestischer Fragenteil}

Die anamnestischen Fragen können von den zu Untersuchenden alleine und selbstständig beantwortet werden. Sie geben dem Behandler die Möglichkeit, sich ein Bild über den Verlauf und die Intensität der von einer CMD ausgelösten Schmerzen zu machen. Außerdem werden erste Fragen gestellt, die dabei helfen sollen, die Ursachen und die Lokalisation der Schmerzen herauszuarbeiten.

\begin{tabular}{|c|c|}
\hline \multicolumn{2}{|c|}{ Anamnestische Angaben des Patienten: } \\
\hline & Wie würden Sie Ihren allgemeinen Gesundheitszustand einschätzen? \\
\hline F 1 & ausgezeichnet \\
\hline
\end{tabular}




\begin{tabular}{|c|c|}
\hline \multirow[b]{2}{*}{ F 2} & Wie würden Sie Ihren Mundgesundheitszustand einschätzen? \\
\hline & 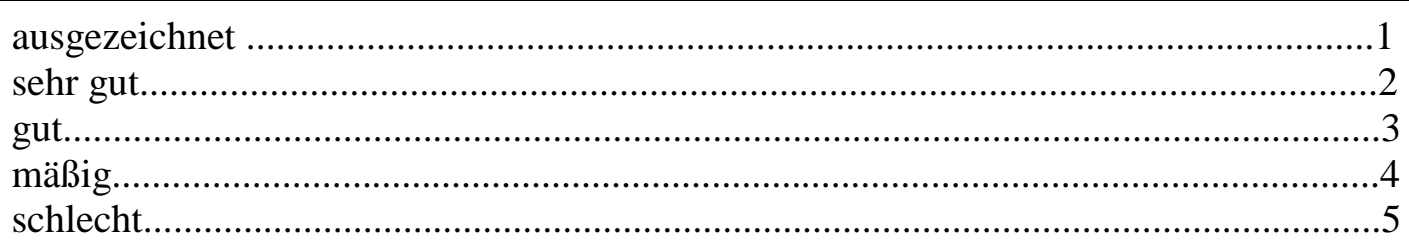 \\
\hline \multirow[b]{2}{*}{ F 3} & $\begin{array}{l}\text { Hatten Sie Schmerzen im Gesicht, dem Kiefer, den Schläfen, vor dem oder im } \\
\text { Ohr im vergangenen Monat? }\end{array}$ \\
\hline & Jann Ja: siehe nächste Frage, sonst weiter mit F 5 \\
\hline \multirow[b]{2}{*}{$\mathbf{F} 4 \mathbf{a}$} & Vor wie vielen Jahren begannen Ihre Gesichtsschmerzen zum ersten Mal? \\
\hline & $\begin{array}{l}\text { Jahre } \\
\text { (Wenn es ein Jahr oder länger her ist, dann weiter zu Frage F 5) } \\
\text { (Wenn es weniger als ein Jahr her ist, dann 00 eintragen.) }\end{array}$ \\
\hline \multirow{2}{*}{$\mathbf{F} \mathbf{4 b}$} & Vor wie vielen Monaten begannen Ihre Gesichtsschmerzen zum ersten Mal? \\
\hline & __ Monate \\
\hline \multirow[b]{2}{*}{ F 5} & Ist Ihr Gesichtsschmerz dauernd, wiederkehrend oder trat er nur einmalig auf? \\
\hline & 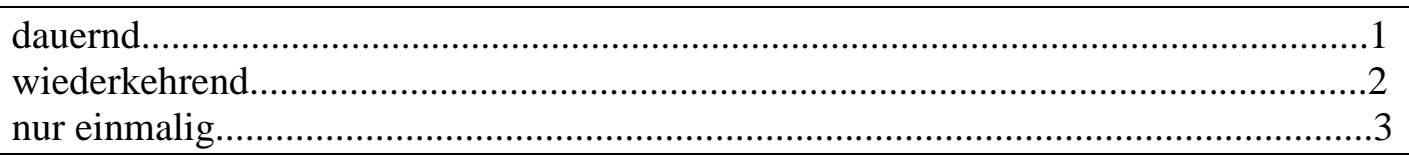 \\
\hline \multirow[b]{2}{*}{ F 6} & $\begin{array}{l}\text { Sind Sie aufgrund der Gesichtsschmerzen zu einem Arzt, Zahnarzt oder zu An- } \\
\text { gehörigen anderer Heilberufe gegangen? }\end{array}$ \\
\hline & 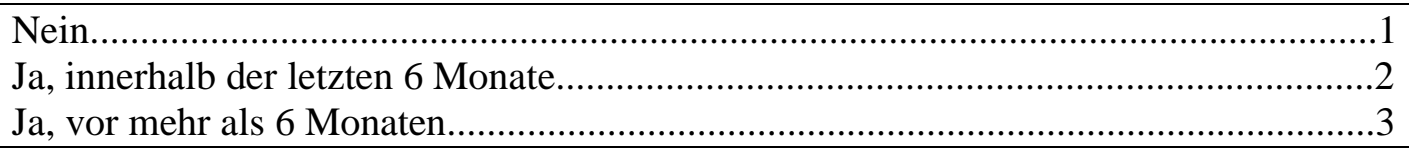 \\
\hline \multirow{2}{*}{ F 14a } & $\begin{array}{l}\text { War Ihr Unterkiefer jemals blockiert oder hatten Sie Schwierigkeiten, den Mund } \\
\text { vollständig zu öffnen? }\end{array}$ \\
\hline & Jann Nein, weiter mit Frage 15) \\
\hline \multirow[t]{2}{*}{ F 14b } & $\begin{array}{l}\text { War diese Mundöffnungsbehinderung so stark, dass dabei Ihre Fähigkeit zu es- } \\
\text { sen beeinflusst war? }\end{array}$ \\
\hline & Nain \\
\hline \multirow{2}{*}{ F 15a } & $\begin{array}{l}\text { Knackt es in Ihrem Kiefergelenk, wenn Sie den Mund öffnen oder schließen oder } \\
\text { wenn Sie kauen? }\end{array}$ \\
\hline & Nein \\
\hline \multirow[t]{2}{*}{ F 15b } & $\begin{array}{l}\text { Nehmen Sie in Ihrem Kiefergelenk ein reibendes Geräusch wahr, wenn Sie den } \\
\text { Mund öffnen oder schließen oder wenn Sie kauen? }\end{array}$ \\
\hline & Nain \\
\hline
\end{tabular}




\begin{tabular}{|c|c|}
\hline \multirow[t]{2}{*}{ F 15c } & $\begin{array}{l}\text { Wurde Ihnen gesagt oder haben Sie selbst bemerkt, dass Sie im Schlaf mit den } \\
\text { Zähnen pressen oder knirschen? }\end{array}$ \\
\hline & Nein \\
\hline \multirow{2}{*}{ F 15d } & Pressen oder knirschen Sie mit den Zähnen am Tage? \\
\hline & Nein \\
\hline \multirow[t]{2}{*}{ F 15e } & $\begin{array}{l}\text { Haben Sie Schmerzen oder ein Gefühl der Steifheit im Kieferbereich beim mor- } \\
\text { gendlichen Erwachen? }\end{array}$ \\
\hline & $\mathrm{N}$ \\
\hline \multirow{2}{*}{ F 15f } & Haben Sie Ohrgeräusche oder Ohrklingen? \\
\hline & Nein \\
\hline \multirow{2}{*}{ F 15g } & Fühlt sich der Zusammenbiss Ihrer Zähne ungewöhnlich oder unbequem an? \\
\hline & Nein \\
\hline \multirow{2}{*}{ F 16a } & $\begin{array}{l}\text { Haben Sie rheumatoide Arthritis, Lupus erythematodes oder eine andere allge- } \\
\text { meine Gelenkerkrankung des Körpers? }\end{array}$ \\
\hline & Nein \\
\hline \multirow{2}{*}{ F 16b } & Hat jemand in Ihrer Familie eine dieser Erkrankungen? \\
\hline & Nein \\
\hline \multirow{2}{*}{ F 16c } & $\begin{array}{l}\text { Hatten oder haben Sie geschwollene oder schmerzhafte Gelenke (das Kieferge- } \\
\text { lenk ausgenommen)? }\end{array}$ \\
\hline & Jann Nein, weiter mit Frage 17a) \\
\hline \multirow{2}{*}{ F 16d } & $\begin{array}{l}\text { Wenn ja, ist das ein anhaltender Schmerz, den Sie schon länger als ein Jahr ha- } \\
\text { ben? }\end{array}$ \\
\hline & Nein \\
\hline \multirow[b]{2}{*}{ F17a } & $\begin{array}{l}\text { Haben Sie in der letzten Zeit eine/n Verletzung/Unfall im Bereich des Kiefers } \\
\text { oder des Gesichtes erlitten? }\end{array}$ \\
\hline & $\begin{array}{l}\text { Ja } \\
\text { (Wenn Nein, weiter mit Frage 18) }\end{array}$ \\
\hline \multirow{2}{*}{ F17b } & Wenn Ja, hatten Sie Ihren Gesichtsschmerz schon vor diesem Ereignis? \\
\hline & . \\
\hline
\end{tabular}




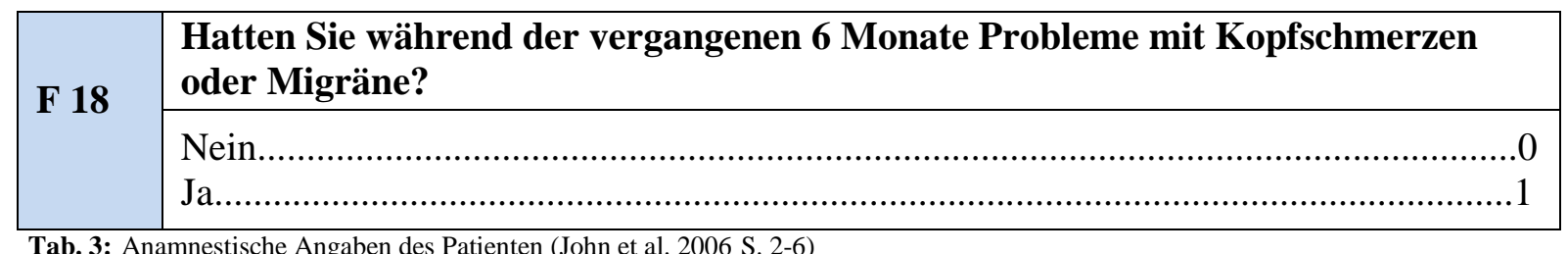

Hier sorgt die RDC/TMD durch ihre Zahlencodierung dafür, dass sich im Anschluss an die Untersuchung durch Auswertung der Bögen der Grad des chronischen Schmerzes klassifizieren lässt.

Dieser Teil des Fragebogens wurde den Probanden vor der Untersuchung vorgelegt und ausgefüllt. Im Nachhinein konnte somit für jeden von ihnen eine Einstufung der vorhandenen Schmerzen vorgenommen werden. Da sich die vorliegende Arbeit weniger mit den Schmerzen, sondern im Schwerpunkt mit den klinischen Symptomen befasst, sind die anamnestischen Fragen zwar für die Auffindung von Symptomen relevant und müssen deshalb an dieser Stelle auch genannt werden, sind aber letztlich von untergeordneter Bedeutung. Außerdem sollte erwähnt werden, dass die Gruppe der Probanden bisher nicht unter schmerzhaften Symptomen einer CMD gelitten hat. Aus dem gleichen Grund schien es auch nicht erforderlich zu sein, die "Jaw disability list" (Dworkin und LeResche 1992) sowie Fragen nach depressiven Erkrankungen in den Fragenkatalog der Probanden mit aufzunehmen. Diese Kriterien spielen nur für Patienten mit einer langen CMD-Vorgeschichte eine signifikante Rolle und sind somit bei schmerzfreien Probanden ohne Relevanz.

\subsubsection{Klinische Untersuchung}

Nachdem die anamnestischen Angaben gemacht worden waren, wurden alle Probanden anhand der Vorgaben des unten aufgeführten Fragebogens befragt und klinisch untersucht. Einige Fragen aus dem anamnestischen Teil der RDC/TMD wurden mit in das klinische Untersuchungsblatt aufgenommen, um es dem Arzt zu ermöglichen, die von Patienten selbst lokalisierten Schmerzen und das von ihnen beschriebene Ausmaß durch eine klinische Untersuchung genauer zu überprüfen und zu verifizieren.

Im Rahmen der Untersuchung wurden folgende Grundregeln beachtet:

1. Alle Messungen werden mit passiver Muskulatur durchgeführt, es sei denn, die Untersuchung besagt etwas anderes. 
2. Alle Millimetermessungen werden auf eine oder zwei Stellen hinter dem Komma gerundet. Liegt eine Messung genau zwischen zwei Werten, ist der kleinere zu Grunde zulegen.

3. Alle Probanden müssen in einem $90^{\circ}$-Winkel zum Behandler sitzen, und zwar am besten in einem Stuhl.

4. Der Behandler trägt zu jeder Zeit der Untersuchung Handschuhe.

5. Prothesen sind während der Untersuchung im Mund zu belassen, es sei denn, die Gingiva - und/oder die Schleimhautverhältnisse müssen überprüft werden.

6. Falls der Proband Bartträger ist oder eine Halskrause trägt, welche die Palpation der Kaumuskulatur behindert, so ist dies zu vermerken.

7. Die Untersuchung erfolgt in der vorgegebenen Reihenfolge.

Für die einzelnen Untersuchungen gelten ferner spezielle Verhaltensregeln, die von den Probanden und dem Behandler einzuhalten sind.

\section{Untersuchungsblatt (Achse II Befunde):}

\begin{tabular}{|l|l|c|}
\hline 1. & Haben Sie Schmerzen in der rechten Gesichtshälfte, in der linken oder in beiden? \\
\cline { 2 - 3 } & keine & 0 \\
& rechts & 1 \\
& links & 2 \\
& beide & 3 \\
\hline
\end{tabular}

Hier wird die entsprechende Antwort angekreuzt. Falls der Proband Schmerzen im Bereich der Mittellinie angibt, wird "beide" angekreuzt.

\begin{tabular}{|c|c|c|c|c|}
\hline \multirow[t]{6}{*}{2.} & \multicolumn{4}{|c|}{$\begin{array}{l}\text { Können Sie auf die schmerzende Stelle zeigen? (Der Untersucher tastet die gezeigte } \\
\text { Stelle ab, wenn unklar ist, ob es Muskel- oder Gelenkschmerzen sind) }\end{array}$} \\
\hline & \multicolumn{2}{|l|}{ rechts } & \multicolumn{2}{|l|}{$\underline{\text { links }}$} \\
\hline & keine & 0 & keine & 0 \\
\hline & Kiefergelenk & 1 & Kiefergelenk & 1 \\
\hline & Muskel & 2 & Muskel & 2 \\
\hline & beides & 3 & beides & 3 \\
\hline
\end{tabular}

Ist unklar, ob es sich an der gezeigten Stelle um Muskel- oder Gelenkschmerzen handelt, muss der Behandler die gezeigte Stelle palpieren. Ist das Ergebnis des Palpierens nicht identisch mit den Angaben des Probanden, wird immer das Ergebnis des Behandlers zugrundegelegt. 


\begin{tabular}{|c|c|c|}
\hline \multirow[t]{2}{*}{3.} & \multicolumn{2}{|l|}{ Mundöffnungsbewegung } \\
\hline & $\begin{array}{l}\text { gerade } \\
\text { seitliche Abweichung (Deflexion) nach rechts } \\
\text { „s"-förmige Abweichung (Deviation) nach rechts } \\
\text { seitliche Abweichung (Deflexion) nach links } 3 \\
\text { „,“-förmige Abweichung (Deviation) nach links } \\
\text { anderes Muster } \\
\text { Typ. }\end{array}$ & $\begin{array}{l}0 \\
1 \\
2 \\
3 \\
4 \\
5\end{array}$ \\
\hline
\end{tabular}

Tab. 6: Untersuchungsblatt (Achse II Befunde): Mundöffnungsbewegung (John et al. 2006 S. 9)

Zunächst sollte der Proband dazu aufgefordert werden, seinen Unterkiefer in eine komfortable Position zu bringen. Dabei können sich die Zähne leicht berühren. Nun sollte der Behandler mit dem Daumen die Unterlippe leicht nach unten bewegen, so dass man die Frontzähne einsehen und auf diese Weise beim Öffnen und Schließen des Mundes die Mittellinie beobachten kann. Nun sollte der Patient den Mund dreimal öffnen. Dabei wird die Mundöffnungsbewegung beobachtet. Ein Lineal kann hier zu Hilfe genommen werden.

\begin{tabular}{|c|c|c|c|c|c|c|}
\hline \multirow[t]{8}{*}{4.} & \multicolumn{6}{|c|}{ Vertikaler Bewegungsumfang } \\
\hline & \multicolumn{6}{|c|}{$\begin{array}{l}\text { a) akt. max. Öffnung ohne Schmerzen ............mm } \\
\text { b) akt. max. Öffnung ............mm } \\
\text { c) passive max. Öffnung } \\
\text { d) Overbite ................mm } \\
\text { e) Overjet ...........mm }\end{array}$} \\
\hline & \multicolumn{3}{|c|}{ Muskelschmerzen } & \multicolumn{3}{|c|}{ Gelenkschmerzen } \\
\hline & & b) & c) & & b) & c) \\
\hline & keine & 0 & 0 & keine & 0 & 0 \\
\hline & rechts & 1 & 1 & rechts & 1 & 1 \\
\hline & links & 2 & 2 & links & 2 & 2 \\
\hline & beide & 3 & 3 & beide & 3 & 3 \\
\hline
\end{tabular}

Tab. 7: Untersuchungsblatt (Achse II Befunde): Vertikaler Bewegungsumfang (John et al. 2006 S. 9)

Bei der Untersuchung zum vertikalen Bewegungsumfang bedeutet „aktiv“, dass der Proband den Mund zunächst alleine öffnet. Bei passiver Öffnung lässt der Behandler den Probanden den Mund soweit wie möglich öffnen, sodann drückt er mit dem Daumen gegen die Oberkieferfrontzähne und mit dem Zeigefinger gegen die Unterkieferfrontzähne. Nun kann er mit steigendem, aber nicht zu starkem Druck die Mundöffnung passiv erweitern, wenn es möglich ist. Alle Werte werden mit dem Lineal gemessen. Dabei sollte sich die Messung auf den Abstand zwischen den Inzisalkanten der jeweiligen Schneidezähne beziehen. Falls das Messergebnis unter $30 \mathrm{~mm}$ liegen sollte, ist dies gesondert zu dokumentieren. Entstehen bei dieser 
Untersuchung Schmerzen, so sind diese getrennt nach Muskel- und Gelenkschmerzen für die rechte, linke oder beide Seiten zu dokumentieren.

Der Overbite stellt die inzisale horizontale Überlappung der Schneidekanten dar, der Overjet die vertikale. Beide werden mit dem Lineal gemessen. Beim Overjet kann eine Hilfslinie am Zahn die Messung verbessern.

\begin{tabular}{|c|c|c|c|}
\hline \multirow[t]{7}{*}{5.} & \multicolumn{3}{|c|}{ Gelenkgeräusche (Palpation) } \\
\hline & a) Öffnung & rechts & $\underline{\text { links }}$ \\
\hline & $\begin{array}{l}\text { keine } \\
\text { Knacken } \\
\text { starkes Reiben } \\
\text { feines Reiben } \\
\text { Öffnungsknacken bei }\end{array}$ & $\begin{array}{c}0 \\
1 \\
2 \\
3 \\
\ldots \ldots \ldots m \\
\end{array}$ & $\begin{array}{c}0 \\
1 \\
2 \\
3 \\
\ldots \ldots \ldots . m m \\
\end{array}$ \\
\hline & \multicolumn{3}{|l|}{ b) Schließen } \\
\hline & $\begin{array}{l}\text { keine } \\
\text { Knacken } \\
\text { starkes Reiben } \\
\text { feines Reiben } \\
\text { Schließungsknacken bei }\end{array}$ & $\begin{array}{c}0 \\
1 \\
2 \\
3 \\
\ldots \ldots m m \\
\end{array}$ & $\begin{array}{c}0 \\
1 \\
2 \\
3 \\
\ldots \ldots \ldots m \\
\end{array}$ \\
\hline & \multicolumn{3}{|l|}{$\begin{array}{l}\text { c) reziprokes Knacken } \\
\text { verhindert bei protrusiver } \\
\text { Öffnung }\end{array}$} \\
\hline & $\begin{array}{l}\text { nein } \\
\text { ja } \\
\text { nicht reproduzierbar }\end{array}$ & $\begin{array}{l}0 \\
1 \\
9\end{array}$ & $\begin{array}{l}0 \\
1 \\
9\end{array}$ \\
\hline
\end{tabular}

Der Proband gibt zunächst an, ob Gelenkgeräusche vorhanden sind. Ist dies der Fall, sollten diese nun durch den Behandler näher untersucht werden. Bei der Untersuchung sollte der Behandler seine Zeigefinger auf der jeweiligen Seite auf das Kiefergelenk legen. Als Orientierung kann hier der Tragus des Ohres dienen, vor welchem der Zeigefinger platziert werden sollte. Daraufhin sollte der Proband gebeten werden, den Mund langsam zu öffnen und zu schließen. Bei Mundschluss sollte immer eine maximale Interkuspidation vorhanden sein. Im weiteren Verlauf der Untersuchung können nun die Befunde in der Tabelle notiert werden. Liegt ein Öffnungs- oder Schließknacken vor, muss der interinzisale Abstand mit einem Lineal gemessen und notiert werden. Dies gilt aber nur für reproduzierbare, also bei mehreren Mundöffnungen und gleichem interinzisalen Abstand sich wiederholende Knackgeräusche.

Ein reziprokes Knacken ist klinisch definiert als reproduzierbares Knacken beim Öffnen und Schließen des Mundes, welches jedoch bei protrudiertem Öffnen und Schließen des Mundes 
nicht mehr wahrzunehmen ist. Falls der Patient kein reproduzierbares Öffnungs- und Schließungsknacken hat, ist 9 anzukreuzen.

\begin{tabular}{|c|c|c|c|c|c|c|c|c|c|c|}
\hline \multirow[t]{6}{*}{6.} & \multicolumn{10}{|c|}{ Bewegungen } \\
\hline & \multirow[t]{2}{*}{ Bewegung } & \multirow[t]{2}{*}{$\begin{array}{l}\text { Bewegung } \\
\text { in } \mathrm{mm}\end{array}$} & \multicolumn{4}{|c|}{ Muskelschmerzen } & \multicolumn{4}{|c|}{ Gelenkschmerzen } \\
\hline & & & $\begin{array}{l}\text { keine } \\
0\end{array}$ & $\begin{array}{l}\text { re. } \\
1\end{array}$ & $\begin{array}{l}\text { li. } \\
2\end{array}$ & $\begin{array}{l}\text { beide } \\
3\end{array}$ & $\begin{array}{l}\text { keine } \\
0\end{array}$ & $\begin{array}{l}\text { re. } \\
1\end{array}$ & $\begin{array}{l}\text { li. } \\
2\end{array}$ & $\begin{array}{l}\text { beide } \\
3\end{array}$ \\
\hline & $\begin{array}{l}\text { Laterotr. } \\
\text { rechts }\end{array}$ & & & & & & & & & \\
\hline & $\begin{array}{l}\text { Laterotr. } \\
\text { links }\end{array}$ & & & & & & & & & \\
\hline & Protrusion & & & & & & & & & \\
\hline
\end{tabular}

Alsdann wird der Proband dazu aufgefordert, seinen Mund soweit wie möglich nach rechts, links bzw. nach vorne zu bewegen. Bei jeder dieser Bewegungen sollte mit einem Lineal der Bewegungsumfang gemessen und festgehalten werden. Nach jeder Bewegung in eine Richtung muss der Behandler gezielt nach Muskel- und/oder Gelenkschmerzen fragen. Um eine Unterscheidung zwischen Muskel- und Gelenkschmerzen möglich zu machen, kann der Behandler gegebenenfalls die schmerzenden Stellen palpieren und so einen eindeutigen Befund bestimmen.

\begin{tabular}{|l|l|l|c|c|}
\hline 7. & \multirow{2}{*}{ Gelenkgeräusche bei Bewegung } & & $\begin{array}{l}\text { Geräusche } \\
\text { rechts }\end{array}$ & $\begin{array}{l}\text { Geräusche } \\
\text { links }\end{array}$ \\
\cline { 2 - 5 } & \multirow{3}{*}{ Bewegung nach rechts } & keine & 0 & 0 \\
& Knacken & 1 & 1 \\
& starkes Reiben & 2 & 2 \\
& feines Reiben & 3 & 3 \\
\hline \multirow{3}{*}{ Bewegung nach links } & keine & 0 & 0 \\
& Knacken & 1 & 1 \\
& starkes Reiben & 2 & 2 \\
\cline { 2 - 4 } & feines Reiben & 3 & 3 \\
\hline \multirow{2}{*}{ Protrusion } & keine & 0 & 0 \\
& Knacken & 1 & 1 \\
& starkes Reiben & 2 & 2 \\
\hline
\end{tabular}

Tab. 10: Untersuchungsblatt (Achse II Befunde): Gelenkgeräusche bei Bewegung (John et al. 2006 S. 10)

Bei den Bewegungen nach rechts, links und vorne sollten auftretende Geräusche ebenfalls dokumentiert werden. Dies ist indessen nur angezeigt, wenn diese reproduzierbar sind. 


\begin{tabular}{|c|c|c|c|c|c|c|c|c|c|}
\hline \multirow[t]{9}{*}{8.} & $\begin{array}{ll}\text { Extraorale Muskelpalpation } \\
\text { kein Schmerz/nur Druck } & =0 \\
\text { leichter Schmerz } & =1 \\
\text { mäßiger Schmerz } & =2 \\
\text { heftiger Schmerz } & =3\end{array}$ & \multicolumn{4}{|c|}{ rechts } & \multicolumn{4}{|c|}{ links } \\
\hline & a) Temporalis posteriorer Teil & 0 & 1 & 2 & 3 & 0 & 1 & 2 & 3 \\
\hline & b) Temporalis medialer Teil & 0 & 1 & 2 & 3 & 0 & 1 & 2 & 3 \\
\hline & c) Temporalis anteriorer Teil & 0 & 1 & 2 & 3 & 0 & 1 & 2 & 3 \\
\hline & d) Masseterursprung & 0 & 1 & 2 & 3 & 0 & 1 & 2 & 3 \\
\hline & e) Masseterkörper & 0 & 1 & 2 & 3 & 0 & 1 & 2 & 3 \\
\hline & f) Masseteransatz & 0 & 1 & 2 & 3 & 0 & 1 & 2 & 3 \\
\hline & g) Regio retromandibularis & 0 & 1 & 2 & 3 & 0 & 1 & 2 & 3 \\
\hline & h) Regio submandibularis & & 1 & 2 & 3 & & 1 & 2 & \\
\hline
\end{tabular}

Tab. 11: Untersuchungsblatt (Achse II Befunde): Extraorale Muskelpalpation (John et al. 2006 S. 11)

Die extraorale Muskelpalpation sollte stets mit definiertem Druck bei leicht geöffnetem Mund und mit dem Zeige- und Mittelfinger zusammen erfolgen, während die andere Hand den Kopf des Patienten auf der nicht zu palpierenden Seite leicht fixiert. Der Druck für die Muskelpalpation sollte ca. $5 \mathrm{~N}$ betragen (Ottl et al. 2005). Dem Probanden sollten vor der Untersuchung die verschiedenen Antwortmöglichkeiten erklärt werden. Die Antworten des Probanden werden entsprechend dokumentiert. Um die Muskeln besser palpieren und Regionen des Muskels mit erhöhter Spannung besser verifizieren zu können, kann der Proband gebeten werden, den Mund zu schließen und dabei fest zuzubeißen.

\begin{tabular}{|l|l|lllll|llll|}
\hline 9. & Palpation des Gelenks & 0 & 1 & 2 & 3 & 0 & 1 & 2 & 3 \\
\cline { 2 - 10 } & a) lateraler Kondylenpol & 0 & 1 & 2 & 3 & 0 & 1 & 2 & 3 \\
\cline { 2 - 9 } & b) posteriorer Kondylenpol &
\end{tabular}

Tab. 12: P Untersuchungsblatt (Achse II Befunde): Palpation des Gelenks (John et al. 2006 S. 11)

Um den lateralen Kondylenpol zu untersuchen, ist der Zeigefinger wieder auf den Tragus vor dem Ohr zu platzieren. Mit einem Druck von ca. 2,5 N kann man das Kiefergelenk nun palpieren. Der posteriore Kondylenpol kann hingegen nur durch Einführen eines Firngers in den äußeren Gehörgang des Patienten palpiert werden. Etwa auftretende Schmerzempfindungen sind zu notieren.

\begin{tabular}{|l|l|lllll|llll|}
\hline 10. & Intraorale Palpation & 0 & 1 & 2 & 3 & 0 & 1 & 2 & 3 \\
\hline & a) Pterygoideus lateralis & 0 & 1 & 2 & 3 & 0 & 1 & 2 & 3 \\
\hline & b) Temporalissehne &
\end{tabular}

Tab. 13: Untersuchungsblatt (Achse II Befunde): Intraorale Palpation (John et al. 2006 S. 11)

Diese Muskeln werden von intraoral mit einem Druck von ca. 2,5 N mit dem Zeigefinger palpiert. Nach dieser Untersuchung ist der klinische Teil beendet und die Gruppe der 30 Proban- 
2. Material und Methoden

den konnte sich im Anschluss daran in mehreren Kleingruppen der Untersuchung im realtime MRT unterziehen. 


\subsection{Methodik der real-time-MRT}

Die Untersuchung der 30 Probanden wurde ausschließlich am Max-Planck-Institut in der Biomedizinischen NMR-Forschungs-GmbH in Göttingen durchgeführt. Dabei wurden sie jeweils am selben Tag, an dem auch die klinische Untersuchung stattgefunden hatte, untersucht. Vor der Untersuchung wurden sie über eventuelle Risiken der MRT-Untersuchung aufgeklärt und sie mussten dies schriftlich bestätigen. Außerdem wurden sie vor Betreten des MRTs über die gültigen Sicherheitsvorschriften unterrichtet und aufgefordert, vorher alle metallischen Gegenstände abzulegen und während der Untersuchung wegen der enormen Geräuschentwicklung Oropax anzulegen.

Die Positionierung der für die Untersuchung notwendigen Spulen konnte nun vorgenommen werden. Dazu wurden die Spulen an die auf dem Untersuchungstisch liegende Person angepasst. Die bilateralen TMJ-Spulen konnten mit Hilfe eines Gestells individuell angepasst werden. Dazu dienten der Tragus und die Frankfurter Horizontalen, welche eine gedachte horizontale Linie durch den tiefstgelegenen Punkt des Unterrandes der Augenhöhle und durch den höchsten Punkt des äußeren knöchernen Gehörgangs darstellt (Lehmann und Hellwig 2005). Mit Hilfe dieser zur Orientierung und durch die Palpation des Kiefergelenks konnte die bestmögliche Position für die Spulen ausgesucht werden. Sie wurden dann so fixiert, dass sie so nah wie möglich am Kiefergelenk der Probanden lagen, aber andererseits auch noch genug Freiraum ließen, damit die Probanden ohne Beeinträchtigung den Mund öffnen und schließen konnten. Soweit sich später bei den ersten Localizer-Aufnahmen herausstellte, dass die Spulen nicht an der richtigen Stelle angebracht waren, wurde deren Position nochmals neu eingestellt. Danach konnte ein eigens angefertigtes Gestell zur Fixierung eines Spiegels über den Augen des Probanden angebracht werden. Dieses war nötig, um ihnen während der MRTUntersuchung über einen Projektor ein Video einzuspielen. Dieses Video bestand aus einer Animation, die einen Mundöffnung und Mundschließung simulierte. Diese Sequenz dauerte $40 \mathrm{sec}$. Auf diese Weise sei eine gleichmäßige Öffnungs- und Schließbewegung des Mundes gewährleistet, um somit zu einer guten Bildqualität beizutragen. Nachdem alle Teile der Spule und des Gestells für die Spiegel eingestellt worden waren, konnte der jeweilige Proband in das MRT gefahren werden.

Die Untersuchung selbst wurde mit einem Magnetresonanztomographen (Tim Trio, Siemens Healthcare, Erlangen) unter Zuhilfenahme der bilateralen Spulen und unter Beachtung der unten aufgeführten Spezifikationen und der aufgelisteten Untersuchungsparameter durchgeführt. 


\begin{tabular}{|c|c|}
\hline real-time-MRT & Technische Spezifikation \\
\hline MRT & 3 T (Tim Trio, Siemens Healthcare, Erlangen) \\
\hline Spulen & $\begin{array}{c}\text { Bilaterale } 2 \times \text { 4-Anordnung von zwei unabhängig und frei } \\
\text { beweglichen 4-Element Spulen( Noras MRT Produkte, } \\
\text { Höchberg) }\end{array}$ \\
\hline Repetition Time (TR) & $4,33 \mathrm{msec}$ \\
\hline Gradient Echo Time (TE) & $2,10 \mathrm{msec}$ \\
\hline Reciever bandwidth & $810 \mathrm{~Hz} /$ pixel \\
\hline Flip angle (RF excitation) & $20^{\circ}$ \\
\hline Field of view (FOV) & $192 \times 192 \mathrm{~mm}$ \\
\hline Base resolution/spoke & 256 data \\
\hline Spokes & 385 \\
\hline Frame rate & 3 Bilder pro Sekunde \\
\hline In-plane resolution & $0,75 \times 0,75 \mathrm{~mm}^{2}$ \\
\hline Section thickness & $5 \mathrm{~mm}$ \\
\hline
\end{tabular}

Tab. 14: Spezifikationen für die Aufnahme des Kiefergelenks mit dem real-time-MRT (Zhang et al. 2010).

Die Untersuchung wurde stets in gleichen Schritten durchgeführt. Dies bedeutet, dass zunächst Localizer-Bilder in koronarer Schnittführung angefertigt wurden. Diese ermöglichten es, die Position der Kiefergelenke genau zu bestimmen und deren Achsausrichtung in der Sagittalen für die folgenden Aufnahmen zu berücksichtigen. Danach konnten von jedem Kiefergelenk erneut je eine Aufnahme mit mehreren Schnitten in der Sagittalen durchgeführt werden, um die zentralste Ansicht als Grundlage für die real-time Aufnahme festzulegen. Diese wurde als zentrale real-time-Aufnahme definiert und auf deren Grundlage wurden eine media- 
le und laterale Aufnahme angefertigt. Das gleiche Prozedere wurde alsdann für die Aufnahmen in einer der Neigung der Kiefergelenkköpfe angepassten Sagittalen verfolgt. Daher ergaben sich pro Proband 17 Aufnahmen, davon 5 Localizer- und 12 real-time-Aufnahmen, wobei jede einzelne real-time-Aufnahme $55 \mathrm{sec}$. gedauert hat. Die gesamte Untersuchungszeit mit Vorbereitung der Probanden kann mit 20 - 30 Minuten veranschlagt werden.

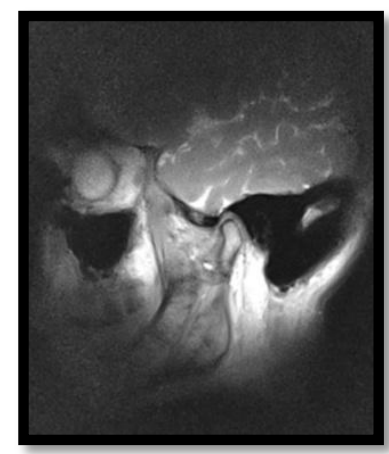

1

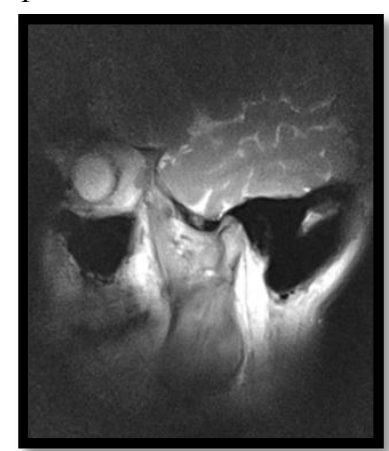

5

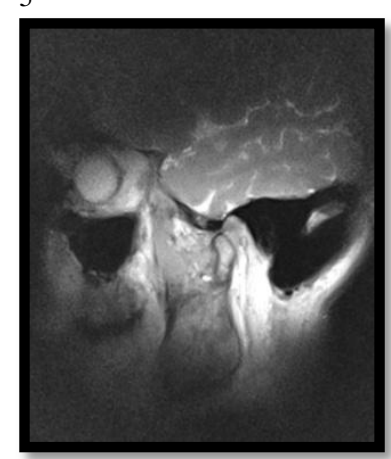

9

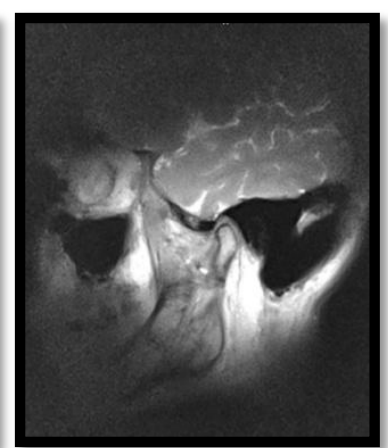

2

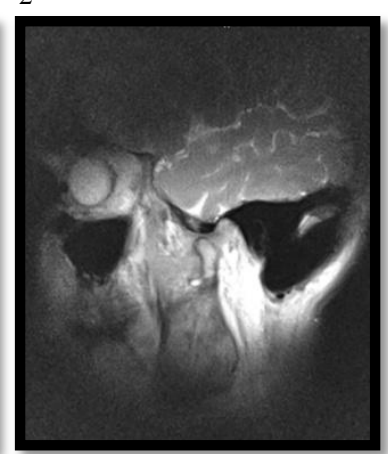

6

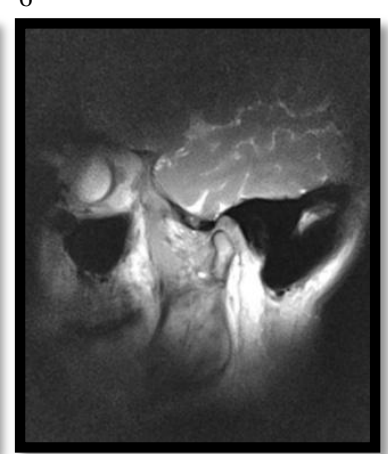

10

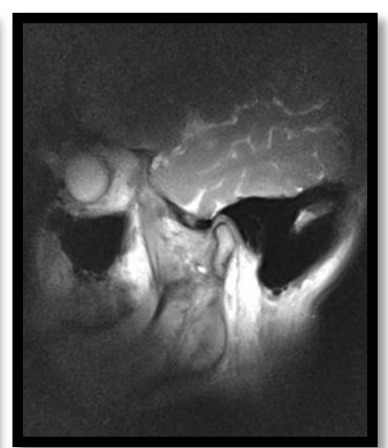

3

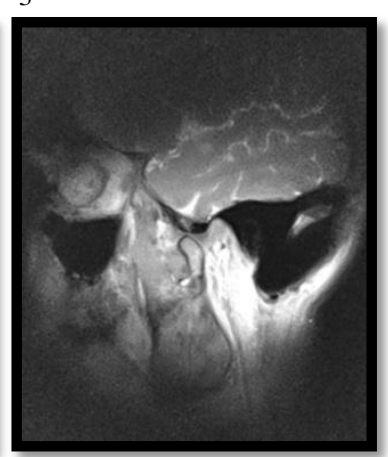

7

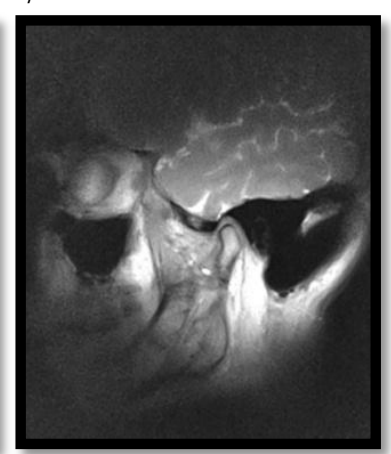

11

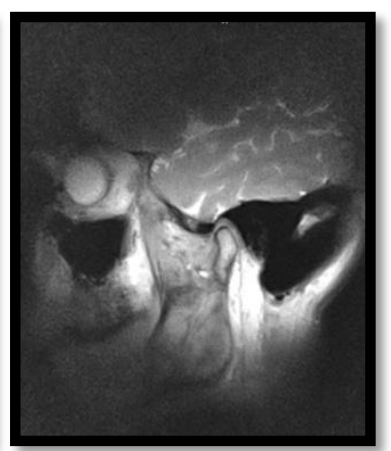

4

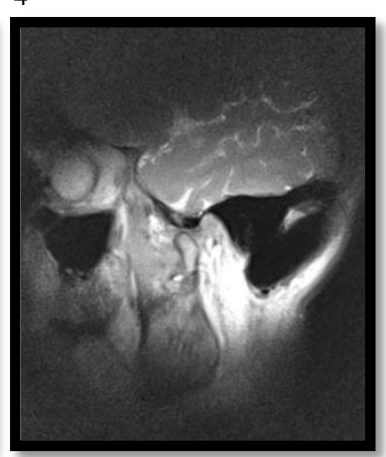

8

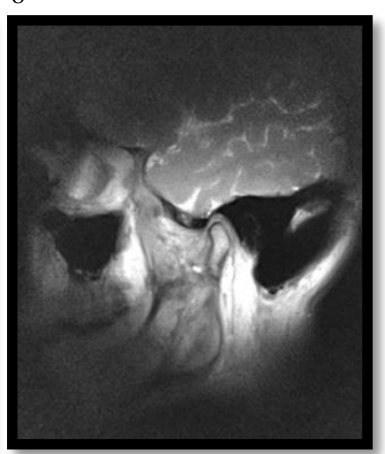

12

Abb.: 9: Bilder 1 - 12 sind exemplarisch aus einer real-time-MRT-Untersuchung ausgewählte Einzelbilder eines Videos, welches die Mundöffnungs- und Mundschlussbewegung wiedergibt. Bild 6 zeigt die maximale Mundöffnung des Probanden. Die Bilder 1 und 12 zeigen den geschlossenen Mund. Die real-time-MRT-Videos bestehen aus 180 Einzelbildern. Da diese nicht alle gezeigt werden können, ist hier nur jedes fünfzehnte Bild abgebildet. Weiterhin ist auf Seite 101 eine CD-ROM mit Beispielvideos hinterlegt. 


\subsection{Auswertung}

Die bei der klinischen Untersuchung gewonnenen Ergebnisse können mit Hilfe der „scoring protocols“ (John et al. 2006) der RDC/TMD vereinheitlicht und in Zahlenwerten veranschaulicht werden. Die Ergebnisse der real-time-MRT wurden unabhängig voneinander durch jeweils drei erfahrene Zahnärzte mit dem Arbeitsschwerpunkt Funktionsdiagnostik und durch drei erfahrene Physiker aus dem Fachbereich der Magnetresonanztomograhie begutachtet und auf die zu erkennenden Details bewertet.

\subsubsection{RDC/TMD}

Eine Ergebnisauswertung der RDC/TMD erfolgte für die Achse I anhand von drei Gruppen:

- Muskelerkrankungen,

- Verlagerung des Discus articularis und

- Arthralgie, Arthritis, Arthrose des Kiefergelenks (John et al. 2006).

Für jede dieser Gruppen existiert ein eigener Berechnungsalgorithmus, durch den jeder Patient individuell in die gegebenen Untergruppen eingeordnet werden kann. Damit lässt sich für jeden Patienten eine genaue, aber immer auf - gleiche Daten und Befundergebnisse begründete - Diagnose stellen.

\subsubsection{Gruppe I. Muskelerkrankungen}

\subsection{Myofaszialer Schmerz (I.a.)}

Er gilt, wenn der Proband Schmerzen im Bereich der Kiefer, des Gesichts, der Schläfen oder der Ohren bei Ruhe oder Funktion angibt. (Eine positive Antwort auf die Frage: Hatten Sie während der letzten Monate Schmerzen im Bereich der Kiefer, des Gesichts, über oder an den Ohren?). Hinzu kommen Schmerzen nach der Palpation von mindesten drei der folgenden 20 Muskelpalpationsstellen:

- M. temporalis posteriore, mittlere und anteriore Region;

- M. masseter Ursprung, Ansatz und Muskelbauch;

- Regio postmandibularis und retromandibularis;

- Region des M. pterygoideus lateralis;

- Sehnen des M. temporalis. 
Dabei gelten die linke und die rechte Seite als separate Taststellen, wobei mindestens eine der Taststellen auf der vom Patienten angegebenen schmerzenden Seite liegen muss.

\subsection{Myofaszialer Schmerz mit eingeschränkter Mundöffnung (I.b.):}

Hier gelten dieselben Kriterien wie unter 1. angegeben. Weiterhin muss eine schmerzfreie aktive Kieferöffnung von weniger als $40 \mathrm{~mm}$ und eine maximale passive Kieferöffnung, die mindestens $5 \mathrm{~mm}$ größer als die schmerzfreie aktive Kieferöffnung ist, möglich sein.

Gruppe: I (Muskelerkrankungen)

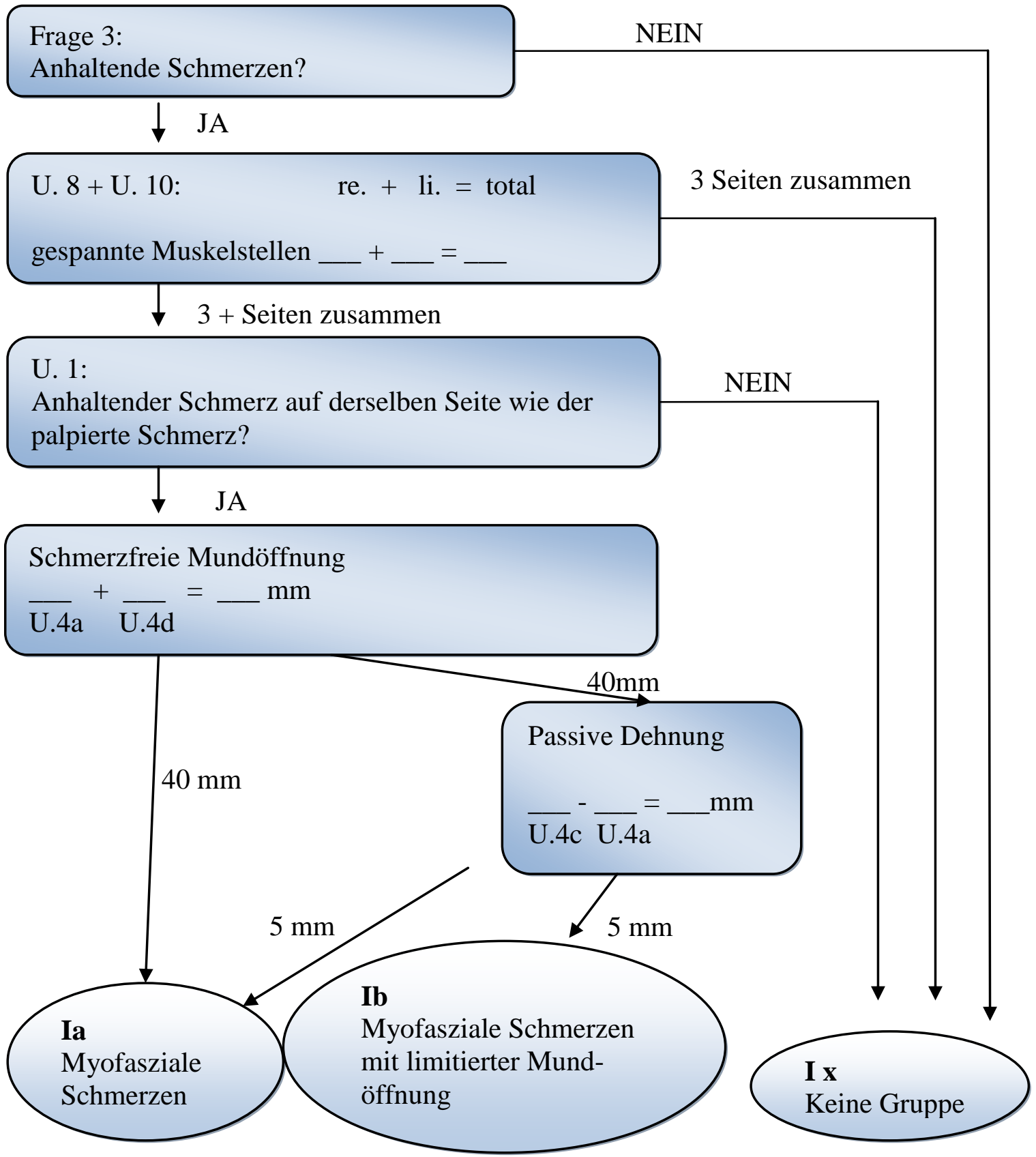

Abb. 10: Gruppe: I (Muskelerkrankungen) (Dworkin und Le Resche 2007 S. 23) 


\subsubsection{Gruppe II. Verlagerung des Discus articularis}

\subsection{Verlagerung des Discus articularis mit Reposition (II.a.)}

Hierbei liegt ein reziprokes Knacken bei zwei von drei Bewegungsdurchläufen der Öffnungs- und Schließbewegung vor. Dabei ist beim Öffnen eine um 5 mm größere Interinzisaldistanz zu erkennen. Außerdem ist das reziproke Knacken durch eine protrusive Kieferposition zu eliminieren. Für diese Untergruppe ist es aber auch zulässig, dass das reziproke Knacken auch bei Lateral- oder Protrusionsbewegungen auftritt.

2.4.1.2.2 Verlagerung des Discus articularis ohne Reposition mit eingeschränkter Kieferöffnung (II.b.)

Diese Diagnose setzt voraus, dass eine eingeschränkte Kieferöffnung in der Anamnese besteht. Auch muss die Kieferöffnung kleiner oder gleich $35 \mathrm{~mm}$ sein. Die maximale passive Kieferöffnung sollte nur $4 \mathrm{~mm}$ oder weniger als die schmerzfreie Kieferöffnung sein. Weiterhin muss die Lateralbewegung eingeschränkt sein und somit unter 7 $\mathrm{mm}$ liegen und / oder es liegt eine unkorrigierte ipsilaterale Deviation beim Öffnen vor. Schließlich dürfen entweder keine Gelenkgeräusche oder aber nur Gelenkgeräusche, die nicht unter 1 . beschrieben sind, oder aber beide vorliegen.

\subsection{Verlagerung des Discus articularis ohne Reposition und ohne eingeschränkte} Kieferöffnung (II.c.)

Für diese Diagnose ist eine eingeschränkte Kieferöffnung in der Anamnese nötig. Hinzu kommt eine schmerzfreie aktive Kieferöffnung von mehr als $35 \mathrm{~mm}$. Dabei muss die maximale passive Kieferöffnung $5 \mathrm{~mm}$ und mehr als die schmerzfreie aktive Kieferöffnung betragen. Weiterhin sind eine eingeschränkte Lateralbewegung von 7 mm und weniger sowie Gelenkgeräusche, die nicht 1. zuzuordnen sind, Voraussetzung. Außerdem können bildgebende Verfahren (Arthrographie, MRT), soweit möglich, zur Diagnosesicherung eingesetzt werden. 
Gruppe: II (Verlagerung des Discus articularis)

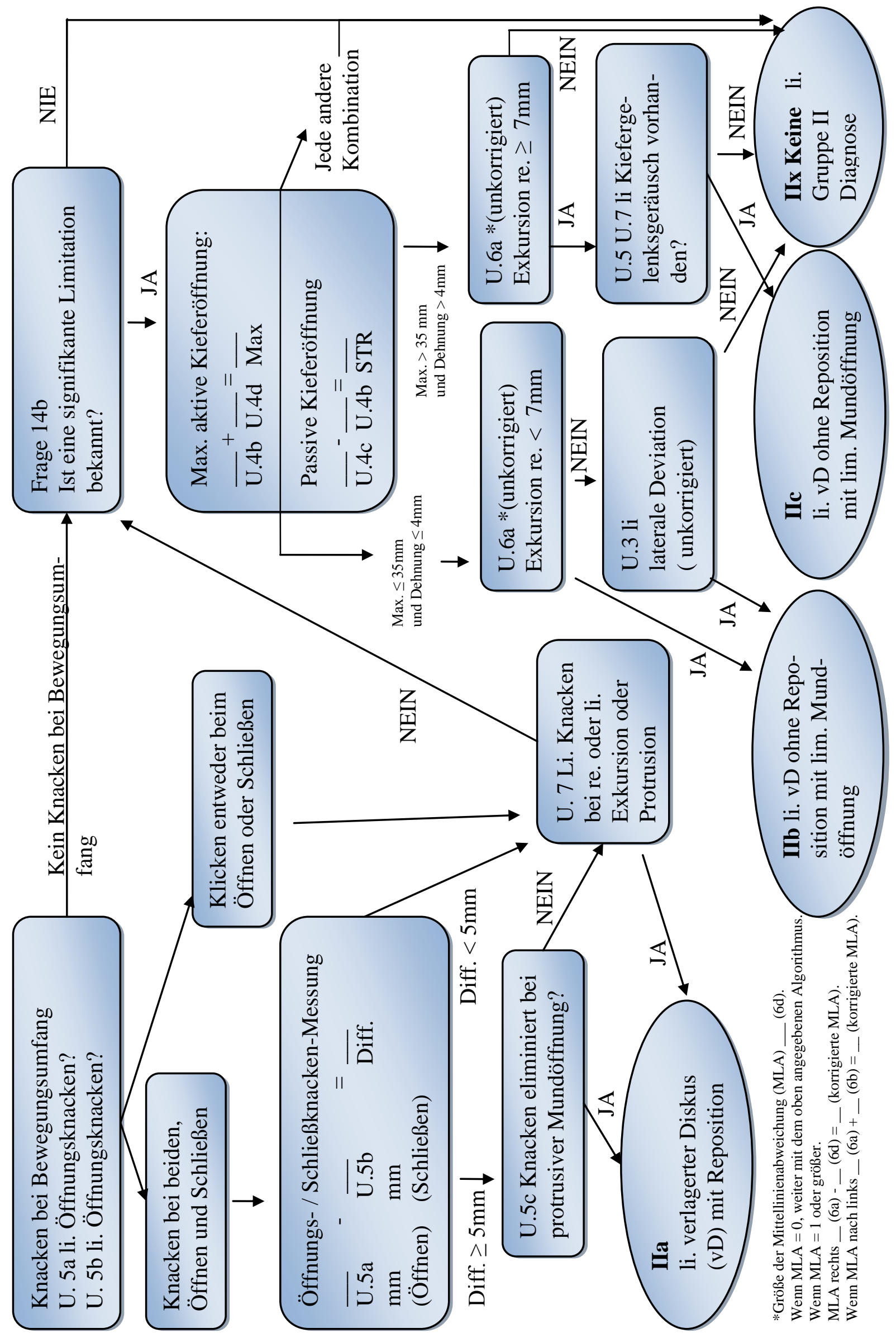

Abb. 11: Gruppe: II (Verlagerung des Discus articularis)Ist genauso auch für das re. Kiefergelenk anwendbar (Dworkin und LeResche 2007 S. 24) 


\subsubsection{Gruppe III. Arthralgie, Arthritis, Arthrose des Kiefergelenks}

\subsection{Arthralgie (III.a.)}

Hier liegen zunächst einmal Schmerzen bei der Palpation (lateral und/oder posterior) in einem oder beiden Kiefergelenken vor. Hinzu kommen noch eine oder mehrere der folgenden Patientenangaben:

- Schmerzen im Kiefergelenksbereich,

- Kiefergelenksschmerzen bei aktiver Kieferöffnung,

- Kiefergelenksschmerzen bei passiver Kieferöffnung,

- Kiefergelenksschmerzen bei Lateralbewegungen.

Hierbei dürfen allerdings keine Krepitationsgeräusche auftreten.

\subsection{Arthritis (III.b.)}

Zuerst sind hier die gleichen Gegebenheiten vorzufinden wie unter 1.. Hinzukommen entweder Krepitationsgeräusche im Kiefergelenk oder aber die Kiefergelenksaufnahmen zeigen einen oder mehrere der folgenden Befunde:

- Erosion der Kortikalis,

- ausgeprägte plane Schlifffläche (an Stellen mechanischer Überbelastung: ventrokranialer Bereich des Condylus mandibulae am dorsalen Abhang des Tuberculum articulare),

- subchondrale Spongiosasklerosierung im Bereich des Condylus mandibulae und Tuberculum articulare,

- Randzacken (Osteophyten).

\subsection{Arthrose (III.c.)}

Es sind keine Schmerzen wie unter 1. zu beobachten. Aber es liegen Krepitationsgeräusche im Kiefergelenk vor und/oder Kiefergelenksaufnahmen zeigen Befunde wie unter 2. 
Gruppe: III (Arthralgie, Arthritis, Arthrose des Kiefergelenks)

Schmerzen bei Palpation

U. 9a, U. 9b Li. Gelenkschmerz bei Palpation

Schmerzempfindung

U. 2 andauernder Schmerz im li. Gelenk oder

U. 4b, U. 4c Schmerzen im li. Gelenk beim Öffnen oder

U. 6a, U. 6b Li. Gelenksschmerzen bei Exkursion

Schmerzen bei Palpation und

Schmerzempfindung

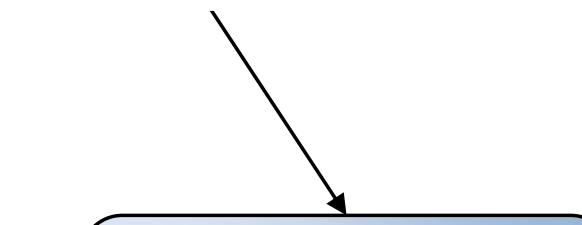

U. 5a, b; 7a, b, c. Krepitus im linken Gelenk während irgendeiner Bewegung?
Weder Schmerzen bei Palpation noch Schmerzempfindung
Entweder Schmerzen bei Palpation oder Schmerzempfindung, aber nicht beides

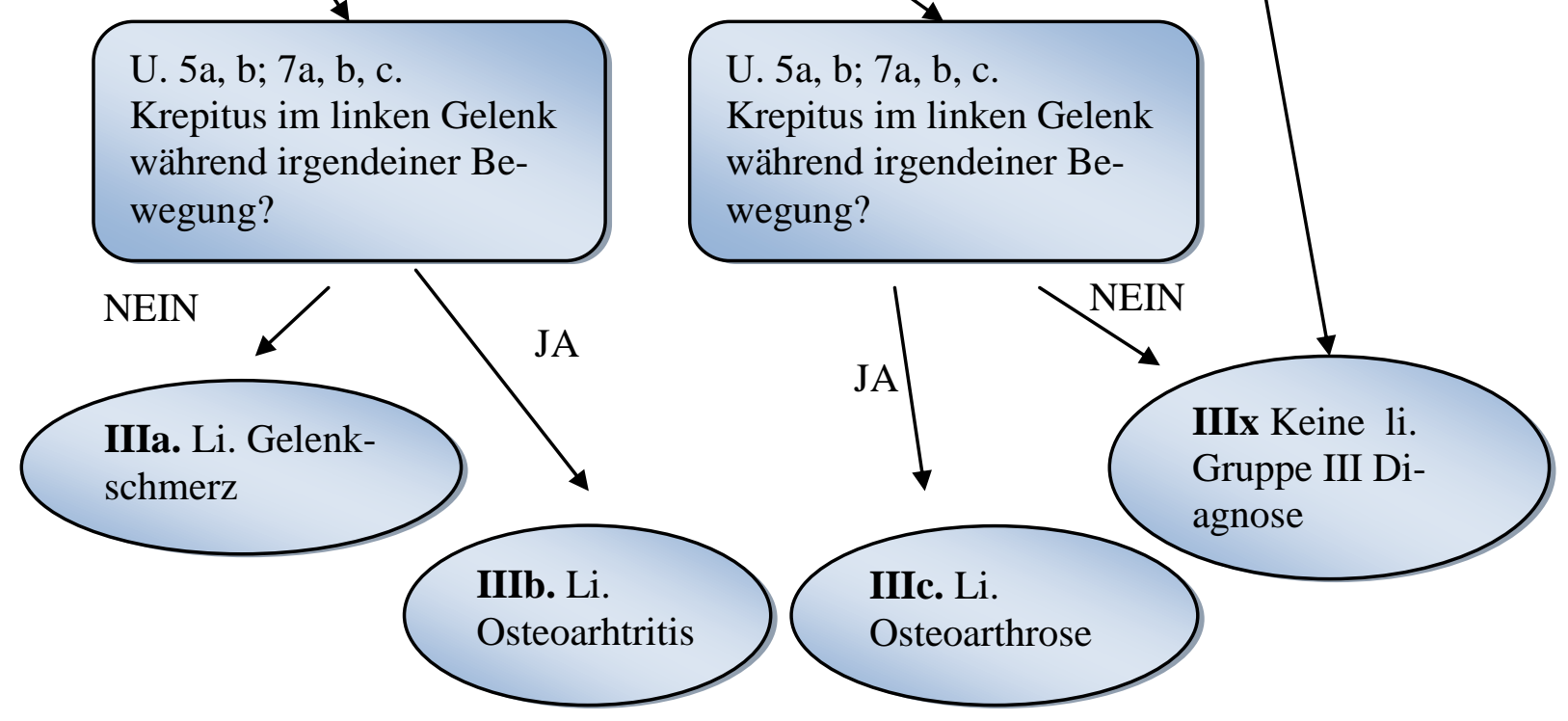

Abb. 12: Gruppe: III (Arthralgie, Arthritis, Arthrose des Kiefergelenks) Ist genauso auch für das re. Kiefergelenk anwendbar (Dworkin und LeResche 2007 S. 26)

So lässt sich auch für die vorliegende Arbeit jeder der 30 Freiwilligen anhand der RDC/TMD in eine der drei Diagnosegruppen und deren Untergruppen einteilen. Damit wird es im Rahmen dieser Arbeit möglich, die Ergebnisse der klinischen Untersuchungen direkt mit den Ergebnissen der real-time-Aufnahme zu vergleichen. In einem weiterem Schritt muss man nun die in den real-time-Aufnahmen zu erkennenden anatomischen Details und teilweise auch die Pathologien mit den Ergebnissen der RDC/TMD vergleichen. 


\subsubsection{Auswertung der real-time-MRT}

Sämtliche real-time-Aufnahmen der Probanden wurden von drei erfahrenen Zahnärzten (A.H., N.G., M.W.) und einem Studenten der Zahnmedizin (O.K.) bewertet. Dabei wurden die Bewertungskriterien für die Diskusverlagerung und für den Bewegungsumfang des Kondylus als wesentliche anatomische und für CMD relevante Merkmale begutachtet. Die jeweilige Bewertung wurde mit einer Zahl nach dem unten angegebenen Muster verschlüsselt.

\begin{tabular}{|c|l|}
\multicolumn{1}{|l|}{ Diskusverlagerung } \\
\hline 1 & normale Diskuslage/keine Verlagerung \\
\hline 2 & Verlagerung des Discus articularis mit Reposition \\
\hline 3 & Verlagerung des Discus articularis ohne Reposition \\
\hline 4 & Verlagerung des Discus articularis nach posterior \\
\hline 5 & Verlagerung des Discus articularis nach lateral \\
\hline 6 & Verlagerung des Discus articularis nach medial \\
\hline 1 & normaler Bewegungsumfang \\
\hline Bewegungsumfang Kondylus
\end{tabular}


Alle Gutachter waren bei der Bewertung der real-time-Aufnahmen alleine und wussten auch nicht, wie die anderen Gutachter die Aufnahmen bewertet hatten. Weiterhin wurden 10 realtime-Untersuchungen mit den Probanden sowie deren Bewertung wiederholt, um später die Ergebnisse abgleichen zu können.

\subsubsection{Cohens Kappa}

Cohens Kappa $(\kappa)$ wurde als statistischer Wert für die Reliabilität zwischen zwei Gutachtern 1960 von Cohen vorgeschlagen (Cohen 1960). Es ist aber auch verwendbar, wenn zwei Gutachter an zwei verschiedenen Orten die gleiche Messmethode verwenden. Dabei lautet die Gleichung für Cohens Kappa:

$$
\kappa=\frac{p_{0}-\mathrm{p} c}{1-\mathrm{pc}}
$$

wobei $p_{0}$ der gemessene Übereinstimmungswert der beiden Gutachter und pc die zufällig erwartete Übereinstimmung ist. Stimmen die Gutachter in allen Urteilen überein, ist $\kappa=1$. Lassen sich aber nur Übereinstimmungen zwischen den beiden Gutachtern feststellen, welche aus rein mathematischer Sicht das Maß des Zufalls nicht überschreiten, ist der Wert von $\kappa=0$ (Gwet 2008).

Es gibt verschiedene Interpretationsmöglickeiten der Werte für Cohens Kappa. Landis und Koch (1977) schlagen folgende Werte vor: $\kappa<0=$,schlechte Übereinstimmung (poor agreement)“, $\kappa$ zwischen 0 und 0,20 = ,geringe (slight) Übereinstimmung““, 0,21-0,40 = ,ausreichende (fair) Übereinstimmung“, 0,41-0,60 = „mittelmäßige (moderate) Übereinstimmung“, 0,61-0,80 = „beachtliche (substantiale) Übereinstimmung“, 0,81-1,00 = ,(fast) vollkommene (almost) perfect Übereinstimmung“(Landis und Koch 1977).

\subsubsection{Sensitivität und Spezifität}

Beide helfen dabei, die Güte eines diagnostischen Verfahrens in eine mathematische Größe zu überführen. Dies dient dazu, die Effizienz diagnostischer Verfahren miteinander vergleichen zu können. 
Die Sensitivität ist definiert als Anteil der kranken Patienten, die auch korrekter weise durch eine Untersuchung als krank diagnostiziert werden konnten. Anhand der Vierfeldertafel lässt sich folgende Formel zur Berechnung ableiten: (\#RP/(\#RP + \#FN)).

Die Spezifität ist definiert als Anteil an gesunden Patienten, die auch korrekterweise durch eine Untersuchung als gesund diagnostiziert werden konnten. Anhand der Vierfeldertafel lässt sich folgende Formel zur Berechnung ableiten: (\#RN/(\#RN + \#FP)) (Hellwig et al. 2006)

\begin{tabular}{|l|l|l|}
\hline & Patient krank (P.k.) & Patient gesund (P.g.) \\
\hline Test positiv (T.p.) & richtig positiv (RP) & falsch positiv (FP) \\
\hline Test negativ (T.n.) & falsch negativ (FN) & richtig negativ (RN) \\
\hline
\end{tabular}

Tab. 16: Vierfeldertafel zur Berechnung der Sensitivität und Spezifität (modifiziert Hellwig et al. 2006 S. 81) 


\section{Ergebnisse}

\subsection{Ergebnisse der RDC/TMD}

Die Ergebnisse der RDC/TMD konnten anhand der Bewertungsalgorythmen aus den Frageund Untersuchungsbögen extrahiert werden. Dabei hat die RDC/TMD Gruppe I die Untergruppen Ia (myofasziale Schmerzen), Ib (myofasziale Schmerzen mit limitierter Mundöffnung) und Ix (keine Gruppe I Diagnose). Die RDC/TMD Gruppe II hat ihrerseits vier Untergruppen: IIa (verlagerter Diskus mit Reposition), IIb (verlagerter Diskus mit Reposition und limitierter Mundöffnung), IIc (verlagerter Diskus ohne Reposition mit limitierter Mundöffnung) und IIx (keine Gruppe II Diagnose). Die RDC/TMD Gruppe III weist die Untergruppen IIIa (Gelenkschmerz), IIIb (Osteoarthritis), IIIc (Osteoarthrose) und IIIx (keine Gruppe III Diagnose) auf.

\begin{tabular}{|c|c|c|c|c|c|}
\hline \multicolumn{6}{|c|}{ Ergebnisse RDC/TMD } \\
\hline Proband & Seite & $\begin{array}{l}\text { MAX/ } \\
\text { STR }\end{array}$ & $\begin{array}{c}\text { RDC/TMD } \\
\text { Gruppe I }\end{array}$ & $\begin{array}{c}\text { RDC/TMD } \\
\text { Gruppe II }\end{array}$ & $\begin{array}{l}\text { RDC/TMD } \\
\text { Gruppe III }\end{array}$ \\
\hline \multirow{2}{*}{5353} & rechts & \multirow{2}{*}{$53 / 2$} & $\mathrm{Ix}$ & IIx & IIIx \\
\hline & links & & $\mathrm{Ix}$ & IIx & IIIx \\
\hline \multirow{2}{*}{5354} & rechts & \multirow{2}{*}{$50 / 2$} & Ix & IIx & IIIx \\
\hline & links & & Ix & IIx & IIIx \\
\hline \multirow{2}{*}{5355} & rechts & \multirow{2}{*}{$43 / 1$} & Ix & IIx & IIIa \\
\hline & links & & Ia & IIa & IIIx \\
\hline \multirow{2}{*}{5356} & rechts & \multirow{2}{*}{$46 / 1$} & Ix & IIx & IIIx \\
\hline & links & & Ix & IIa & IIIx \\
\hline \multirow{2}{*}{5373} & rechts & \multirow{2}{*}{$59 / 0$} & Ix & IIx & IIIx \\
\hline & links & & Ix & IIx & IIIx \\
\hline \multirow{2}{*}{5374} & rechts & \multirow{2}{*}{$44 / 2$} & Ix & IIa & IIIx \\
\hline & links & & Ix & IIa & IIIx \\
\hline \multirow{2}{*}{5375} & rechts & \multirow{2}{*}{$40 / 3$} & $\mathrm{Ix}$ & IIx & IIIx \\
\hline & links & & Ix & IIx & IIIx \\
\hline \multirow{2}{*}{5376} & rechts & \multirow{2}{*}{$42 / 1$} & $\mathrm{Ia}$ & IIx & IIIx \\
\hline & links & & $\mathrm{Ia}$ & IIx & IIIx \\
\hline \multirow{2}{*}{5377} & rechts & \multirow{2}{*}{$44 / 1$} & Ix & IIx & IIIx \\
\hline & links & & Ix & IIx & IIIx \\
\hline \multirow{2}{*}{5378} & rechts & \multirow{2}{*}{$47 / 1$} & Ix & IIa & IIIx \\
\hline & links & & Ix & IIa & IIIx \\
\hline \multirow{2}{*}{5395} & rechts & \multirow{2}{*}{$51 / 1$} & Ix & IIx & IIIx \\
\hline & links & & Ix & IIx & IIIx \\
\hline
\end{tabular}




\begin{tabular}{|c|c|c|c|c|c|}
\hline 5396 & $\begin{array}{l}\text { rechts } \\
\text { links }\end{array}$ & $43 / 4$ & $\begin{array}{l}\text { Ix } \\
\text { Ix }\end{array}$ & $\begin{array}{l}\text { IIx } \\
\text { IIx }\end{array}$ & $\begin{array}{l}\text { IIIx } \\
\text { IIIx }\end{array}$ \\
\hline 5397 & $\begin{array}{l}\text { rechts } \\
\text { links }\end{array}$ & $61 / 0$ & $\begin{array}{l}\text { Ix } \\
\text { Ix }\end{array}$ & $\begin{array}{l}\text { IIx } \\
\text { IIx }\end{array}$ & $\begin{array}{l}\text { IIIx } \\
\text { IIIx }\end{array}$ \\
\hline 5398 & $\begin{array}{l}\text { rechts } \\
\text { links }\end{array}$ & $45 / 1$ & $\begin{array}{l}\text { Ia } \\
\text { Ia }\end{array}$ & $\begin{array}{l}\text { IIa } \\
\text { IIx }\end{array}$ & $\begin{array}{l}\text { IIIx } \\
\text { IIIx }\end{array}$ \\
\hline 5411 & $\begin{array}{l}\text { rechts } \\
\text { links }\end{array}$ & $55 / 2$ & $\begin{array}{l}\text { Ix } \\
\text { Ix }\end{array}$ & $\begin{array}{l}\text { IIx } \\
\text { IIx }\end{array}$ & $\begin{array}{l}\text { IIIx } \\
\text { IIIx }\end{array}$ \\
\hline 5412 & $\begin{array}{l}\text { rechts } \\
\text { links }\end{array}$ & $54 / 2$ & $\begin{array}{l}\text { Ix } \\
\text { Ix }\end{array}$ & $\begin{array}{l}\text { IIx } \\
\text { IIx }\end{array}$ & $\begin{array}{l}\text { IIIx } \\
\text { IIIx }\end{array}$ \\
\hline 5413 & $\begin{array}{l}\text { rechts } \\
\text { links }\end{array}$ & $42 / 2$ & $\begin{array}{l}\text { Ix } \\
\text { Ix }\end{array}$ & $\begin{array}{l}\text { IIx } \\
\text { IIx }\end{array}$ & $\begin{array}{l}\text { IIIx } \\
\text { IIIx }\end{array}$ \\
\hline 5414 & $\begin{array}{l}\text { rechts } \\
\text { links }\end{array}$ & $44 / 1$ & $\begin{array}{l}\text { Ix } \\
\text { Ix }\end{array}$ & $\begin{array}{l}\text { IIx } \\
\text { IIx }\end{array}$ & $\begin{array}{l}\text { IIIx } \\
\text { IIIX }\end{array}$ \\
\hline 5415 & $\begin{array}{l}\text { rechts } \\
\text { links }\end{array}$ & $50 / 1$ & $\begin{array}{l}\text { Ix } \\
\text { Ix }\end{array}$ & $\begin{array}{l}\text { IIx } \\
\text { IIx }\end{array}$ & $\begin{array}{l}\text { IIIx } \\
\text { IIIx }\end{array}$ \\
\hline 5534 & $\begin{array}{l}\text { rechts } \\
\text { links }\end{array}$ & $49 / 1$ & $\begin{array}{l}\text { Ix } \\
\text { Ix }\end{array}$ & $\begin{array}{l}\text { IIx } \\
\text { IIx }\end{array}$ & $\begin{array}{l}\text { IIIx } \\
\text { IIIx }\end{array}$ \\
\hline 5535 & $\begin{array}{l}\text { rechts } \\
\text { links }\end{array}$ & $43 / 1$ & $\begin{array}{l}\text { Ix } \\
\text { Ix }\end{array}$ & $\begin{array}{l}\text { IIa } \\
\text { IIa }\end{array}$ & $\begin{array}{l}\text { IIIx } \\
\text { IIIx }\end{array}$ \\
\hline 5536 & $\begin{array}{l}\text { rechts } \\
\text { links }\end{array}$ & $49 / 1$ & $\begin{array}{l}\text { Ix } \\
\text { Ix }\end{array}$ & $\begin{array}{l}\text { IIx } \\
\text { IIx }\end{array}$ & $\begin{array}{l}\text { IIIx } \\
\text { IIIx }\end{array}$ \\
\hline 5537 & $\begin{array}{l}\text { rechts } \\
\text { links }\end{array}$ & $50 / 1$ & $\begin{array}{l}\text { Ix } \\
\text { Ix }\end{array}$ & $\begin{array}{l}\text { IIx } \\
\text { IIx }\end{array}$ & $\begin{array}{l}\text { IIIx } \\
\text { IIIx }\end{array}$ \\
\hline 5538 & $\begin{array}{l}\text { rechts } \\
\text { links }\end{array}$ & $50 / 2$ & $\begin{array}{l}\text { Ix } \\
\text { Ix }\end{array}$ & $\begin{array}{l}\text { IIa } \\
\text { IIa }\end{array}$ & $\begin{array}{l}\text { IIIx } \\
\text { IIIx }\end{array}$ \\
\hline 5539 & $\begin{array}{l}\text { rechts } \\
\text { links }\end{array}$ & $48 / 2$ & $\begin{array}{l}\text { Ix } \\
\text { Ix }\end{array}$ & $\begin{array}{l}\text { IIx } \\
\text { IIa }\end{array}$ & $\begin{array}{l}\text { IIIx } \\
\text { IIIx }\end{array}$ \\
\hline 5560 & $\begin{array}{l}\text { rechts } \\
\text { links }\end{array}$ & $46 / 2$ & $\begin{array}{l}\text { Ix } \\
\text { Ix }\end{array}$ & $\begin{array}{l}\text { IIx } \\
\text { IIx }\end{array}$ & $\begin{array}{l}\text { IIIx } \\
\text { IIIx }\end{array}$ \\
\hline 5561 & $\begin{array}{l}\text { rechts } \\
\text { links }\end{array}$ & $47 / 1$ & $\begin{array}{l}\text { Ix } \\
\text { Ix }\end{array}$ & $\begin{array}{l}\text { IIx } \\
\text { IIx }\end{array}$ & $\begin{array}{l}\text { IIIx } \\
\text { IIIx }\end{array}$ \\
\hline 5580 & $\begin{array}{l}\text { rechts } \\
\text { links }\end{array}$ & $49 / 1$ & $\begin{array}{l}\text { Ix } \\
\text { Ix }\end{array}$ & $\begin{array}{l}\text { IIx } \\
\text { IIx }\end{array}$ & $\begin{array}{l}\text { IIIx } \\
\text { IIIx }\end{array}$ \\
\hline 5591 & $\begin{array}{l}\text { rechts } \\
\text { links }\end{array}$ & $43 / 4$ & $\begin{array}{l}\text { Ix } \\
\text { Ix }\end{array}$ & $\begin{array}{l}\text { IIx } \\
\text { IIx }\end{array}$ & $\begin{array}{l}\text { IIIx } \\
\text { IIIx }\end{array}$ \\
\hline 5599 & $\begin{array}{l}\text { rechts } \\
\text { links }\end{array}$ & $51 / 1$ & $\begin{array}{l}\text { Ix } \\
\text { Ix }\end{array}$ & $\begin{array}{l}\text { IIx } \\
\text { IIx }\end{array}$ & $\begin{array}{l}\text { IIIx } \\
\text { IIIx }\end{array}$ \\
\hline
\end{tabular}

Tab. 17: Ergebnisse der RDC/TMD nach Auswertung der Frage- und Untersuchungsbögen anhand der entsprechenden Bewertungsalgorithmen. MAX = Maximale aktive Mundöffnung $(\mathrm{mm})$, STR $($ Stretch $)=$ Maximale passive Mundöffnung $(\mathrm{mm}), \quad$ Ia $=$ Myofasziale Schmerzen, Ib = Myofasziale Schmerzen mit limitierter Mundöffnung, Ix = Keine Gruppe I Diagnose, IIa = verlagerter Diskus mit Reposition, Ilb = verlagerter Diskus ohne Reposition mit limitierter Mundöffnung, IIc = verlagerter Diskus ohne Reposition mit limitierter Mundöffnung, IIx = keine Gruppe II Diagnose, IIIa = Gelenkschmerz, IIIb =. Osteoarthritis, IIIc = Osteoarthrose, IIIx = Keine Gruppe III Diagnose

Die aus der Tabelle 14 gewonnenen Daten lassen sich im Diagramm 1 gut veranschaulichen. Nach den Vorgaben der RDC/TMD, wonach jedes Kiefergelenk in jeder Untergruppe einzeln untersucht wird, erschien es auch sinnvoll, jedes Kiefergelenk einzeln zu dokumentieren und 
auszuwerten. So haben von den 30 Probanden, also 60 untersuchten Kiefergelenken, 55 keine RDC/TMD Gruppe I Diagnose, fünf weisen myofasziale Schmerzen auf während, myofasziale Schmerzen mit limitierter Mundöffnung bei dieser Gruppe von Probanden nicht auftraten. Bei der RDC/TMD Gruppe II weisen 48 der 60 Kiefergelenke keine Gruppe II Diagnose auf. 10 Kiefergelenke haben einen verlagerten Diskus mit Reposition. Diskusverlagerungen ohne Reposition konnten bei keinem der Kiefergelenke diagnostiziert werden. In der RDC/TMD Gruppe III befanden sich 59 Kiefergelenke ohne Gruppe III Diagnose. 1 Kiefergelenk wies Gelenkschmerzen auf. Die Diagnose Osteoarthritis und Osteoarthrose konnte überhaupt nicht gestellt werden.

Außerdem wurde die maximale aktive Mundöffnung (MAX) und die passive Kieferöffnung (STR) mit aufgelistet, um zu zeigen, dass bei keinem der Probanden eine limitierte Kieferöffnung vorlag.

\section{Ergebnisse RDC/TMD}

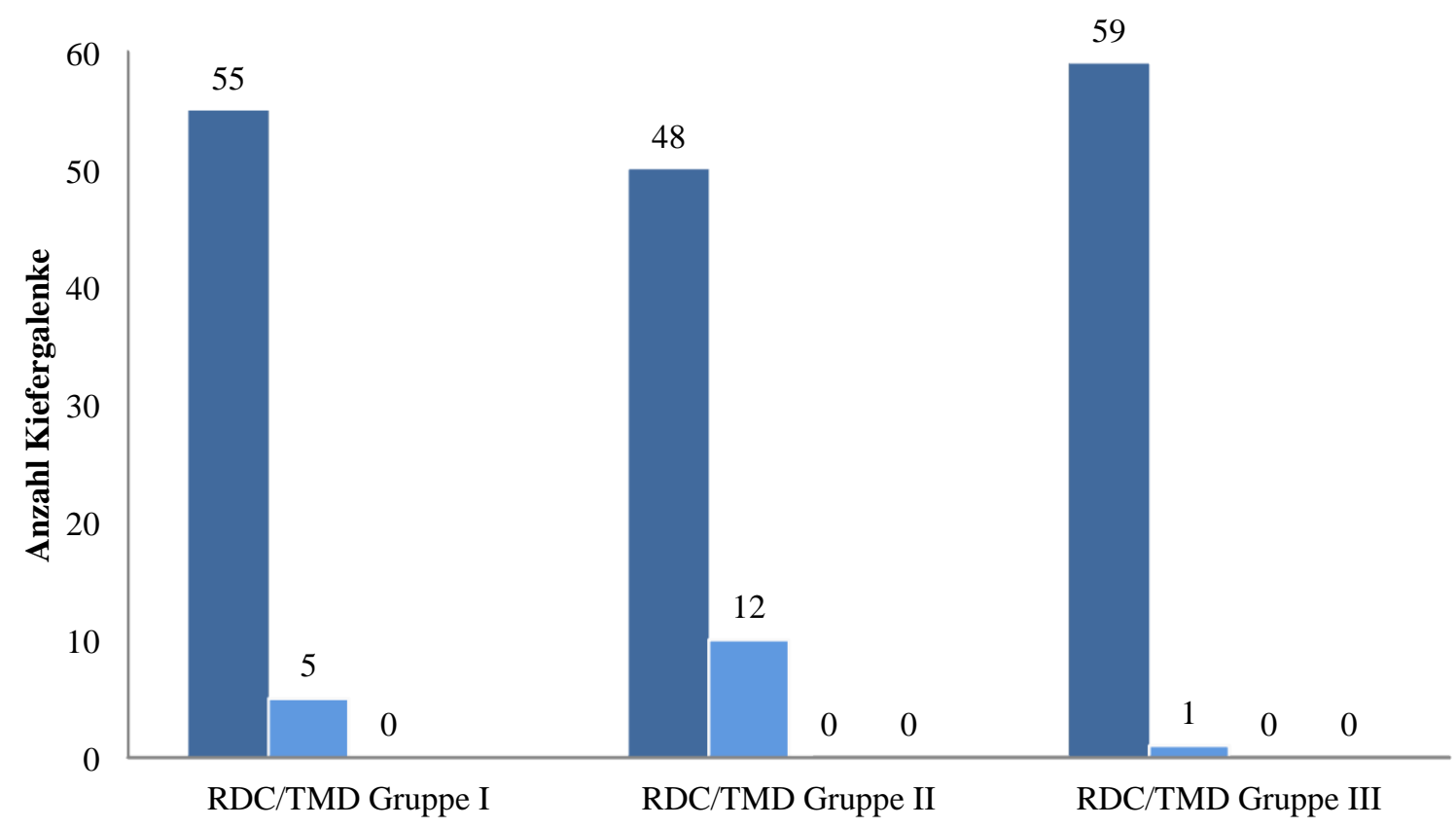

Diagramm 1: Auswertung der RDC/TMD-Untersuchung und Darstellung der Anzahl der gefundenen Diagnosen bezogen auf jedes einzelne Kiefergelenk.

Hier zeigt sich deutlich, dass die Gruppe von Probanden zu jungen Erwachsenen gehört, welche nicht an einer CMD leiden. Es hat zwar ein großer Teil der Probanden Symptome einer CMD, die in den Frage- und Untersuchungsbögen der RDC/TMD vermerkt sind, aber nicht automatisch auch zu einer Diagnose führen. So haben rund 30\% der Probanden (9) eine auf die Bewertungsalgorithmen der RDC/TMD zurückzuführende Diagnose, von denen aber keine der Behandlung bedarf. Dies zeigt, dass wir es mit gesunden Probanden zu tun haben. 


\subsection{Ergebnisse der real-time-MRT}

Die Ergebnisse der real-time-MRT-Untersuchungen konnten von den Gutachtern anhand der vorgegebenen Zahlencodierung bewertet werden. Diese finden sich hier in der Tabelle wieder. Dabei wurde jedes Kiefergelenk einzeln bewertet, um einen direkten Vergleich zwischen den Ergebnissen der RDC/TMD und der real-time-MRT herstellen zu können.

\section{Ergebnisse real-time-MRT}

\begin{tabular}{|c|c|c|c|c|c|c|}
\hline Proband & Seite & $\begin{array}{c}\text { Kiefergelenk } \\
\text { Funktion }\end{array}$ & $\begin{array}{c}\text { Gutachter } \\
1\end{array}$ & $\begin{array}{c}\text { Gutachter } \\
2\end{array}$ & $\begin{array}{c}\text { Gutachter } \\
3\end{array}$ & $\begin{array}{c}\text { Gutachter } \\
4\end{array}$ \\
\hline \multirow{4}{*}{5353} & \multirow{2}{*}{ rechts } & Diskusverl. & 1 & 1 & 1 & 1 \\
\hline & & BU Kondylus & 1 & 1 & 1 & 1 \\
\hline & \multirow{2}{*}{ links } & Diskusverl. & 1 & 1 & 1 & 1 \\
\hline & & BU Kondylus & 1 & 1 & 1 & 1 \\
\hline \multirow{4}{*}{5354} & \multirow{2}{*}{ rechts } & Diskusverl. & 1 & 1 & 1 & 1 \\
\hline & & BU Kondylus & 1 & 1 & 1 & 1 \\
\hline & \multirow{2}{*}{ links } & Diskusverl. & 1 & 1 & 1 & 1 \\
\hline & & BU Kondylus & 1 & 1 & 1 & 1 \\
\hline \multirow{4}{*}{5355} & \multirow{2}{*}{ rechts } & Diskusverl. & 1 & 1 & 1 & 1 \\
\hline & & BU Kondylus & 3 & 3 & 3 & 3 \\
\hline & \multirow{2}{*}{ links } & Diskusverl. & 0 & 0 & 2 & 2 \\
\hline & & BU Kondylus & 3 & 3 & 3 & 3 \\
\hline \multirow{4}{*}{5356} & \multirow{2}{*}{ rechts } & Diskusverl. & 2 & 1 & 1 & 1 \\
\hline & & BU Kondylus & 3 & 3 & 1 & 3 \\
\hline & \multirow{2}{*}{ links } & Diskusverl. & 2 & 2 & 2 & 2 \\
\hline & & BU Kondylus & 3 & 3 & 3 & 3 \\
\hline \multirow{4}{*}{5373} & \multirow{2}{*}{ rechts } & Diskusverl. & 1 & 1 & 1 & 1 \\
\hline & & BU Kondylus & 3 & 3 & 3 & 3 \\
\hline & \multirow{2}{*}{ links } & Diskusverl. & 1 & 1 & 1 & 1 \\
\hline & & BU Kondylus & 1 & 3 & 3 & 1 \\
\hline \multirow{4}{*}{5374} & \multirow{2}{*}{ rechts } & Diskusverl. & 2 & 2 & 2 & 2 \\
\hline & & BU Kondylus & 3 & 3 & 3 & 3 \\
\hline & \multirow{2}{*}{ links } & Diskusverl. & 2 & 2 & 2 & 2 \\
\hline & & BU Kondylus & 3 & 3 & 3 & 3 \\
\hline \multirow{4}{*}{5375} & \multirow{2}{*}{ rechts } & Diskusverl. & 1 & 1 & 1 & 1 \\
\hline & & BU Kondylus & 1 & 2 & 2 & 1 \\
\hline & \multirow{2}{*}{ links } & Diskusverl. & 1 & 1 & 1 & 1 \\
\hline & & BU Kondylus & 1 & 1 & 1 & 1 \\
\hline \multirow{4}{*}{5376} & \multirow{2}{*}{ rechts } & Diskusverl. & 1 & 1 & 1 & 1 \\
\hline & & BU Kondylus & 1 & 1 & 1 & 1 \\
\hline & \multirow{2}{*}{ links } & Diskusverl. & 2 & 1 & 1 & 2 \\
\hline & & BU Kondylus & 3 & 1 & 1 & 1 \\
\hline
\end{tabular}




\begin{tabular}{|c|c|c|c|c|c|c|}
\hline \multirow{4}{*}{5377} & \multirow{2}{*}{ rechts } & Diskusverl. & 1 & 1 & 1 & 1 \\
\hline & & BU Kondylus & 1 & 3 & 3 & 3 \\
\hline & \multirow{2}{*}{ links } & Diskusverl. & 2 & 1 & 2 & 1 \\
\hline & & BU Kondylus & 3 & 3 & 3 & 3 \\
\hline \multirow{4}{*}{5378} & \multirow{2}{*}{ rechts } & Diskusverl. & 2 & 2 & 2 & 2 \\
\hline & & BU Kondylus & 3 & 3 & 3 & 3 \\
\hline & \multirow{2}{*}{ links } & Diskusverl. & 2 & 2 & 2 & 2 \\
\hline & & BU Kondylus & 3 & 3 & 3 & 3 \\
\hline \multirow{4}{*}{5395} & \multirow{2}{*}{ links } & Diskusverl. & 1 & 1 & 1 & 1 \\
\hline & & BU Kondylus & 1 & 1 & 1 & 1 \\
\hline & \multirow{2}{*}{ rechts } & Diskusverl. & 1 & 1 & 1 & 1 \\
\hline & & BU Kondylus & 1 & 1 & 1 & 1 \\
\hline \multirow{4}{*}{5396} & \multirow{2}{*}{ rechts } & Diskusverl. & 1 & 1 & 1 & 1 \\
\hline & & BU Kondylus & 1 & 1 & 1 & 1 \\
\hline & \multirow{2}{*}{ links } & Diskusverl. & 2 & 1 & 2 & 1 \\
\hline & & BU Kondylus & 3 & 3 & 3 & 3 \\
\hline \multirow{4}{*}{5397} & \multirow{2}{*}{ rechts } & Diskusverl. & 1 & 1 & 1 & 1 \\
\hline & & BU Kondylus & 1 & 1 & 1 & 1 \\
\hline & \multirow{2}{*}{ links } & Diskusverl. & 1 & 1 & 1 & 1 \\
\hline & & BU Kondylus & 1 & 1 & 1 & 1 \\
\hline \multirow{4}{*}{5398} & \multirow{2}{*}{ rechts } & Diskusverl. & 1 & 1 & 1 & 1 \\
\hline & & BU Kondylus & 1 & 1 & 1 & 1 \\
\hline & \multirow{2}{*}{ links } & Diskusverl. & 1 & 1 & 1 & 1 \\
\hline & & BU Kondylus & 1 & 1 & 1 & 1 \\
\hline \multirow{4}{*}{5411} & \multirow{2}{*}{ rechts } & Diskusverl. & 1 & 1 & 1 & 1 \\
\hline & & BU Kondylus & 1 & 1 & 1 & 1 \\
\hline & \multirow{2}{*}{ links } & Diskusverl. & 1 & 1 & 1 & 1 \\
\hline & & BU Kondylus & 1 & 1 & 1 & 1 \\
\hline \multirow{4}{*}{5412} & roohts & Diskusverl. & 1 & 1 & 1 & 1 \\
\hline & rechts & BU Kondylus & 1 & 1 & 1 & 1 \\
\hline & $\operatorname{link}_{\mathrm{s}}$ & Diskusverl. & 1 & 1 & 1 & 1 \\
\hline & links & BU Kondylus & 1 & 1 & 1 & 1 \\
\hline & & Diskusverl. & 1 & 1 & 1 & 1 \\
\hline 5413 & rechts & BU Kondylus & 1 & 1 & 1 & 1 \\
\hline 5413 & links & Diskusverl. & 1 & 1 & 1 & 1 \\
\hline & links & BU Kondylus & 1 & 1 & 1 & 1 \\
\hline & & Diskusverl. & 1 & 1 & 1 & 1 \\
\hline 5414 & rechts & BU Kondylus & 1 & 1 & 1 & 1 \\
\hline 5414 & & Diskusverl. & 1 & 1 & 1 & 1 \\
\hline & links & BU Kondylus & 1 & 1 & 1 & 1 \\
\hline & rechts & Diskusverl. & 1 & 1 & 1 & 1 \\
\hline 5415 & rechts & BU Kondylus & 1 & 3 & 3 & 3 \\
\hline 5415 & $\operatorname{link}_{\mathrm{s}}$ & Diskusverl. & 1 & 1 & 1 & 1 \\
\hline & links & BU Kondylus & 1 & 3 & 3 & 1 \\
\hline & & Diskusverl. & 1 & 1 & 1 & 1 \\
\hline 5524 & rechts & BU Kondylus & 1 & 1 & 1 & 1 \\
\hline 5334 & & Diskusverl. & 1 & 1 & 1 & 1 \\
\hline & links & BU Kondylus & 1 & 1 & 1 & 1 \\
\hline
\end{tabular}




\begin{tabular}{|c|c|c|c|c|c|c|}
\hline \multirow{4}{*}{5535} & \multirow{2}{*}{ rechts } & Diskusverl. & 1 & 1 & 1 & 1 \\
\hline & & BU Kondylus & 1 & 1 & 1 & 1 \\
\hline & \multirow{2}{*}{ links } & Diskusverl. & 2 & 1 & 2 & 2 \\
\hline & & BU Kondylus & 3 & 1 & 3 & 1 \\
\hline \multirow{4}{*}{5536} & \multirow{2}{*}{ rechts } & Diskusverl. & 1 & 1 & 1 & 1 \\
\hline & & BU Kondylus & 1 & 1 & 1 & 1 \\
\hline & \multirow{2}{*}{ links } & Diskusverl. & 1 & 1 & 1 & 1 \\
\hline & & BU Kondylus & 1 & 1 & 1 & 1 \\
\hline \multirow{4}{*}{5537} & \multirow{2}{*}{ rechts } & Diskusverl. & 1 & 1 & 1 & 1 \\
\hline & & BU Kondylus & 1 & 1 & 1 & 1 \\
\hline & \multirow{2}{*}{ links } & Diskusverl. & 2 & 1 & 1 & 1 \\
\hline & & BU Kondylus & 3 & 1 & 1 & 1 \\
\hline \multirow{4}{*}{5538} & \multirow{2}{*}{ rechts } & Diskusverl. & 2 & 2 & 2 & 2 \\
\hline & & BU Kondylus & 3 & 3 & 3 & 3 \\
\hline & \multirow{2}{*}{ links } & Diskusverl. & 2 & 2 & 2 & 2 \\
\hline & & BU Kondylus & 3 & 3 & 3 & 3 \\
\hline \multirow{4}{*}{5539} & \multirow{2}{*}{ rechts } & Diskusverl. & 1 & 1 & 1 & 1 \\
\hline & & BU Kondylus & 1 & 1 & 1 & 1 \\
\hline & \multirow{2}{*}{ links } & Diskusverl. & 2 & 2 & 2 & 2 \\
\hline & & BU Kondylus & 3 & 3 & 3 & 3 \\
\hline \multirow{4}{*}{5560} & \multirow{2}{*}{ rechts } & Diskusverl. & 1 & 1 & 1 & 1 \\
\hline & & BU Kondylus & 1 & 1 & 1 & 1 \\
\hline & \multirow{2}{*}{ links } & Diskusverl. & 2 & 2 & 2 & 2 \\
\hline & & BU Kondylus & 3 & 3 & 3 & 3 \\
\hline \multirow{4}{*}{5561} & \multirow{2}{*}{ rechts } & Diskusverl. & 1 & 1 & 1 & 1 \\
\hline & & BU Kondylus & 1 & 1 & 1 & 1 \\
\hline & \multirow{2}{*}{ links } & Diskusverl. & 1 & 1 & 1 & 1 \\
\hline & & BU Kondylus & 1 & 1 & 1 & 1 \\
\hline \multirow{4}{*}{5580} & \multirow{2}{*}{ rechts } & Diskusverl. & 3 & 3 & 3 & 2 \\
\hline & & BU Kondylus & 3 & 3 & 3 & 3 \\
\hline & \multirow{2}{*}{ links } & Diskusverl. & 2 & 2 & 2 & 2 \\
\hline & & BU Kondylus & 3 & 3 & 3 & 3 \\
\hline \multirow{4}{*}{5591} & \multirow{2}{*}{ rechts } & Diskusverl. & 1 & 1 & 1 & 1 \\
\hline & & BU Kondylus & 1 & 1 & 1 & 1 \\
\hline & links & Diskusverl. & 1 & 1 & 1 & 1 \\
\hline & IInKS & BU Kondylus & 1 & 1 & 1 & 1 \\
\hline & & Diskusverl. & 1 & 1 & 1 & 1 \\
\hline & recnts & BU Kondylus & 1 & 1 & 1 & 1 \\
\hline 5599 & & Diskusverl. & 1 & 1 & 1 & 1 \\
\hline & IInKS & BU Kondylus & 1 & 1 & 1 & 1 \\
\hline
\end{tabular}

Tab. 18: Ergebnisse der real-time-MRT-Aufnahme nach der Bewertung durch vier Gutachter. Diskusverl. = Diskusverlagerung, BU = Bewegungsumfang, 0 = nicht beurteilbar, 1 = normale Diskuslage/keine Verlagerung, 2 = Verlagerung des Discus articularis mit Reposition, 3 = Verlagerung des Discus articularis ohne Reposition, 4 = Verlagerung des Discus articularis nach posterior, $5=$ Verlagerung des Discus articularis nach lateral, $6=$ Verlagerung des Discus articularis nach medial

Hierbei hat sich gezeigt, dass die Bewertung der Gutachter nur in 16 der 120 Fälle voneinander abweicht. In allen anderen Fällen stimmen die Gutachter miteinander überein. Aus statistischer Sicht ergeben sich für die Übereinstimmung der Gutachter folgende Werte: 
- Für den Befund „keine Diskusverlagerung“ ergab sich für $\kappa$ ein durchschnittlicher Wert von 0,844 und ein prozentualer Wert von $90 \%$.

- Für den Befund „Diskusverlagerung mit Reposition“ ergab sich für $\kappa$ ein durchschnittlicher Wert von 0,849 und ein prozentualer Wert von $90 \%$.

- Für den Befund „Diskusverlagerung ohne Reposition“ konnte $\kappa$ aus mathematischen Gründen nicht zwischen allen Gutachtern berechnet werden.

- Für den Befund „,normaler Bewegungsumfang des Kondylus“ ließ sich im Durchschnitt ein $\kappa$-Wert von 0,809 und eine prozentuale Übereinstimmung von $85 \%$ berechnen.

- Für den Befund „limitierter Bewegungsumfang des Kondylus“ konnte $\kappa$ aus mathematischen Gründen auch nicht zwischen allen Gutachtern berechnet werden.

- Für den Befund „nicht kontinuierlicher Bewegungsumfang des Kondylus“ konnte ein durchschnittlicher $\kappa$-Wert von 0,835 und eine prozentuale Übereinstimmung von $86 \%$ berechnet werden.

Besser veranschaulicht werden die Ergebnisse in den folgenden Diagrammen. Dazu wurde jede einzelne Bewertung durch einen Gutachter als Ordinate verwendet, um jede mögliche Pathologie wahrnehmen zu können. Somit lässt sich jede einzelne Bewertung eines Gutachters und nicht deren Gesamturteil eines Kiefergelenkes festhalten. Diese Ergebnisse dürfen dabei nicht auf die gesamte Gruppe bezogen werden, da dies sonst zu falschen Rückschlüssen führen würde.

\section{Diskusverlagerung}

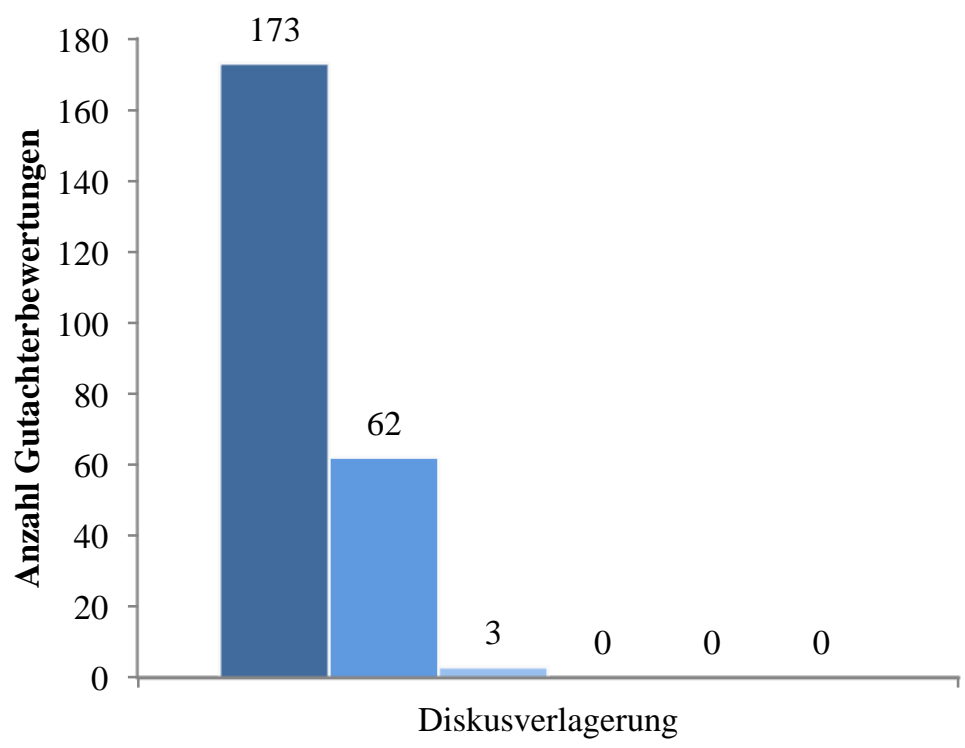

- Normale Diskuslage/keine Verlagerung

- Verlagerung des Diskus articularis mit Reposition

- Verlagerung des Diskus articularis ohne Reposition

- Verlagerung des Diskus articularis nach posterior

- Verlagerung des Diskus articularis nach lateral

- Verlagerung des Diskus articularis nach medial

Diagramm 2: Auswertung der Ergebnisse der real-time-MRT-Aufnahmen für die Formen der Diskusverlagerungen 
Nur bei einem Probanden und zwei Gutachtern war es der Fall, dass aufgrund von technischen Mängeln keine Bewertung vorgenommen werden konnte (in der Tabelle mit „,0“ gekennzeichnet). Diese sind vermutlich auf Bewegungsartefakte zurückzuführen und entstehen dann, wenn der Proband die Kieferbewegung zu ruckartig durchführt. Da aber zwei der vier Gutachter diesen Kiefer nicht mit „0 “sondern mit „2“ bewerteten, kann es auch möglich sein, dass die ruckartigen Bewegungen des Kiefers durch die Reposition des Kondylus auf den Diskus und somit durch die Pathologie selbst entstanden und nicht $\mathrm{zu}$ vermeiden waren. So sind in den Diagrammen 2 und 3 nur 238 Bewertungspunkte angegeben anstatt 240. Es zeigt sich, dass der Befund „normale Diskuslage/keine Verlagerung“ mit 173 Punkten mit Abstand am häufigsten ist. Darauf folgt mit 62 Bewertungspunkten die „Verlagerung des Discus articularis mit Reposition“ und mit drei Punkten die „Verlagerung des Discus articularis ohne Reposition“. Eine Verlagerung des Discus articularis nach posterior, lateral oder medial konnte bei keinem Probanden festgestellt werden.

\section{Bewegungsumfang des Kondylus}

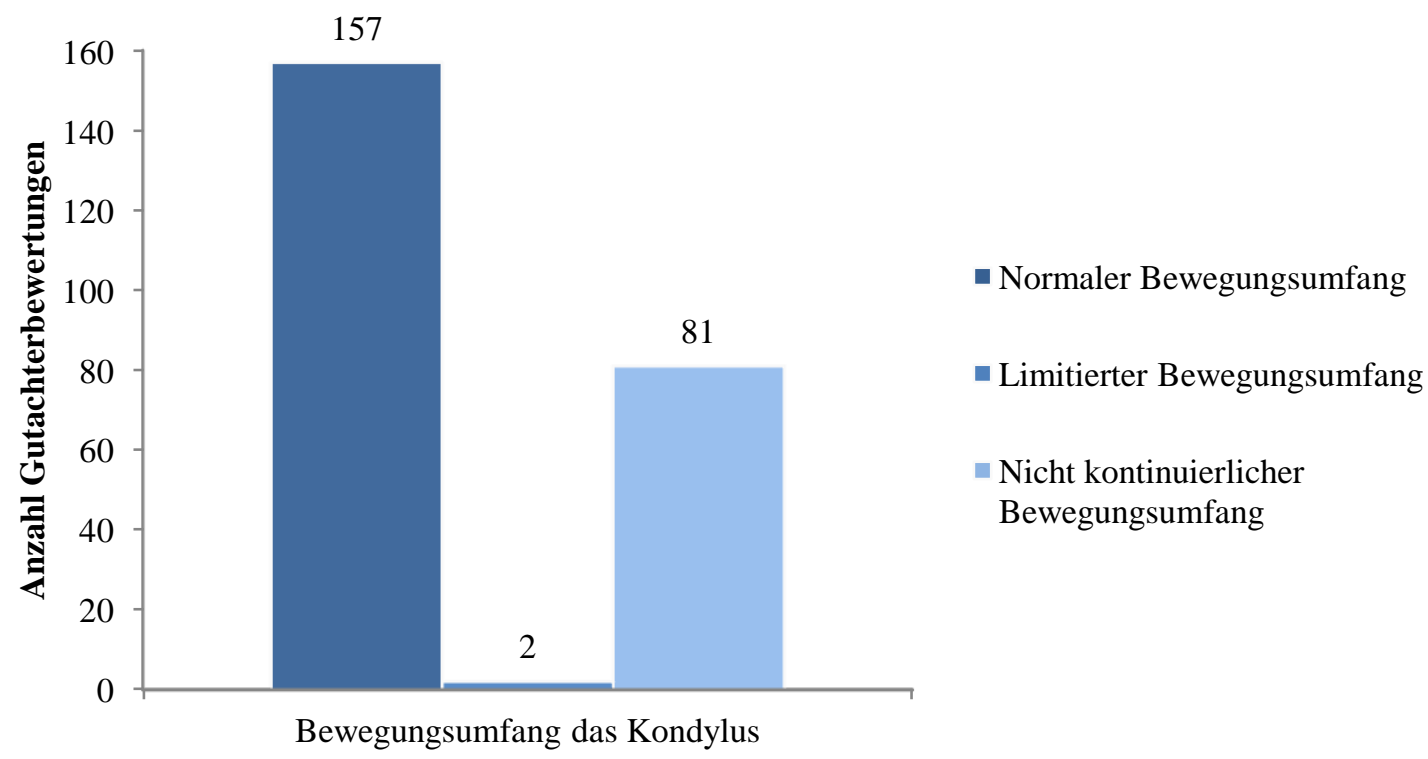

Diagramm 3: Auswertung der Ergebnisse der real-time-MRT-Aufnahmen für den Bewegungsumfang der Kondylen

Als zweiter Parameter wurde von den Gutachtern der Bewegungsumfang des Kondylus bewertet. Dabei konnten die Ergebnisse, wie auch bei der Diskusverlagerung, mit Zahlen für entsprechende Befunde codiert werden. Dabei ergaben 157 Befunde eine normalen Bewegungsumfang des Kondylus. Davon waren zwei limitiert und 81 wurden als nicht kontinuierlich bewertet. 


\subsection{Ergebnisse der Nachuntersuchung}

Weiterhin wurden 10 Probanden zu einem späteren Zeitpunkt erneut mit der real-time-MRT untersucht. Die Auswertung der Ergebnisse durch dieselben Gutachter wurde auch erneut unter den gleichen Rahmenbedingungen wie bei der Erstuntersuchung durchgeführt. Die Ergebnisse der Erstuntersuchung und der Nachuntersuchung werden in Tabelle 16 gegenübergestellt.

\begin{tabular}{|c|c|c|c|c|c|c|c|c|c|c|}
\hline \multicolumn{11}{|c|}{ Vergleich der Ergebnisse des real-time-MRT und deren Nachuntersuchung } \\
\hline Proband & Seite & $\begin{array}{l}\text { Kiefergelenk } \\
\text { Funktion }\end{array}$ & G1 & G2 & G3 & G4 & $\begin{array}{l}\text { G1 } \\
\mathbf{N}\end{array}$ & $\begin{array}{l}\text { G2 } \\
\mathbf{N}\end{array}$ & $\begin{array}{l}\text { G3 } \\
\text { N }\end{array}$ & $\begin{array}{c}\text { G4 } \\
\text { N }\end{array}$ \\
\hline \multirow{4}{*}{5353} & \multirow{2}{*}{ rechts } & Diskusverl. & 1 & 1 & 1 & 1 & 1 & 1 & 1 & 1 \\
\hline & & BU Kondylus & 1 & 1 & 1 & 1 & 1 & 1 & 1 & 1 \\
\hline & \multirow{2}{*}{ links } & Diskusverl. & 1 & 1 & 1 & 1 & 1 & 1 & 1 & 1 \\
\hline & & BU Kondylus & 1 & 1 & 1 & 1 & 1 & 1 & 1 & 1 \\
\hline \multirow{4}{*}{5354} & \multirow{2}{*}{ rechts } & Diskusverl. & 1 & 1 & 1 & 1 & 1 & 1 & 1 & 1 \\
\hline & & BU Kondylus & 1 & 1 & 1 & 1 & 1 & 1 & 1 & 1 \\
\hline & \multirow{2}{*}{ links } & Diskusverl. & 1 & 1 & 1 & 1 & 1 & 1 & 1 & 1 \\
\hline & & BU Kondylus & 1 & 1 & 1 & 1 & 1 & 1 & 1 & 1 \\
\hline \multirow{4}{*}{5356} & \multirow{2}{*}{ rechts } & Diskusverl. & 2 & 1 & 1 & 1 & 2 & 1 & 1 & 1 \\
\hline & & BU Kondylus & 3 & 3 & 1 & 3 & 3 & 3 & 1 & 3 \\
\hline & \multirow{2}{*}{ links } & Diskusverl. & 2 & 2 & 2 & 2 & 2 & 2 & 2 & 2 \\
\hline & & BU Kondylus & 3 & 3 & 3 & 3 & 3 & 3 & 3 & 3 \\
\hline \multirow{4}{*}{5377} & \multirow{2}{*}{ rechts } & Diskusverl. & 1 & 1 & 1 & 1 & 1 & 1 & 1 & 1 \\
\hline & & BU Kondylus & 1 & 3 & 3 & 3 & 1 & 3 & 3 & 3 \\
\hline & \multirow{2}{*}{ links } & Diskusverl. & 2 & 1 & 2 & 1 & 2 & 1 & 2 & 1 \\
\hline & & BU Kondylus & 3 & 3 & 3 & 3 & 3 & 3 & 3 & 3 \\
\hline \multirow{4}{*}{5378} & \multirow{2}{*}{ rechts } & Diskusverl. & 2 & 2 & 2 & 2 & 2 & 2 & 2 & 2 \\
\hline & & BU Kondylus & 3 & 3 & 3 & 3 & 3 & 3 & 3 & 3 \\
\hline & \multirow{2}{*}{ links } & Diskusverl. & 2 & 2 & 2 & 2 & 2 & 2 & 2 & 2 \\
\hline & & BU Kondylus & 3 & 3 & 3 & 3 & 3 & 3 & 3 & 3 \\
\hline \multirow{4}{*}{5396} & \multirow{2}{*}{ rechts } & Diskusverl. & 1 & 1 & 1 & 1 & 1 & 1 & 1 & 1 \\
\hline & & BU Kondylus & 1 & 1 & 1 & 1 & 1 & 1 & 1 & 1 \\
\hline & \multirow{2}{*}{ links } & Diskusverl. & 2 & 1 & 2 & 1 & 2 & 1 & 2 & 2 \\
\hline & & BU Kondylus & 3 & 3 & 3 & 3 & 3 & 3 & 3 & 3 \\
\hline \multirow{4}{*}{5397} & \multirow{2}{*}{ rechts } & Diskusverl. & 1 & 1 & 1 & 1 & 1 & 1 & 1 & 1 \\
\hline & & BU Kondylus & 1 & 1 & 1 & 1 & 1 & 1 & 1 & 1 \\
\hline & \multirow{2}{*}{ links } & Diskusverl. & 1 & 1 & 1 & 1 & 1 & 1 & 1 & 1 \\
\hline & & BU Kondylus & 1 & 1 & 1 & 1 & 1 & 1 & 1 & 1 \\
\hline \multirow{4}{*}{5411} & \multirow{2}{*}{ rechts } & Diskusverl. & 1 & 1 & 1 & 1 & 1 & 1 & 1 & 1 \\
\hline & & BU Kondylus & 1 & 1 & 1 & 1 & 1 & 1 & 1 & 1 \\
\hline & \multirow{2}{*}{ links } & Diskusverl. & 1 & 1 & 1 & 1 & 1 & 1 & 1 & 1 \\
\hline & & BU Kondylus & 1 & 1 & 1 & 1 & 1 & 1 & 1 & 1 \\
\hline
\end{tabular}




\begin{tabular}{|c|c|c|c|c|c|c|c|c|c|c|}
\hline \multirow{3}{*}{5534} & \multirow{3}{*}{ rechts } & Diskusverl. & 1 & 1 & 1 & 1 & 1 & 1 & 1 & 1 \\
\cline { 3 - 11 } & & BU Kondylus & 1 & 1 & 1 & 1 & 1 & 1 & 1 & 1 \\
\cline { 3 - 11 } & \multirow{2}{*}{ links } & Diskusverl. & 1 & 1 & 1 & 1 & 1 & 1 & 1 & 1 \\
\cline { 3 - 11 } & & BU Kondylus & 1 & 1 & 1 & 1 & 3 & 1 & 1 & 1 \\
\hline \multirow{3}{*}{5537} & \multirow{2}{*}{ rechts } & Diskusverl. & 1 & 1 & 1 & 1 & 1 & 1 & 1 & 1 \\
\cline { 3 - 11 } & \multirow{2}{*}{ links } & BU Kondylus & 1 & 1 & 1 & 1 & 1 & 1 & 1 & 1 \\
\cline { 3 - 11 } & & Diskusverl. & 2 & 1 & 1 & 1 & 2 & 1 & 1 & 1 \\
\cline { 3 - 10 } & & BU Kondylus & 3 & 1 & 1 & 1 & 3 & 1 & 1 & 1 \\
\hline
\end{tabular}

Tab.: 19 Vergleich der Ergebnisse der real-time-MRT und deren Nachuntersuchungen. Diskusverl. = Diskusverlagerung, BU = Bewegungsumfang, 0 = nicht beurteilbar, 1 = normale Diskuslage/keine Verlagerung, 2 = Verlagerung des Discus articularis mit Reposition, 3 = Verlagerung des Discus articularis ohne Reposition, 4 = Verlagerung des Discus articularis nach posterior, $5=$ Verlagerung des Discus articularis nach lateral, 6 = Verlagerung des Discus articularis nach medial, G 1-4 = Gutachter 1-4, G 1-4 N = Gutachter 1-4 Nachuntersuchung

Dazu ist festzustellen, dass die Übereinstimmung unter den Gutachtern sehr hoch ist. Von 400 Einzelbefunden jeden Gutachters stimmen nur zwei in der Nachuntersuchung nicht überein. Danach stimmte die Nachuntersuchung mit der Erstuntersuchung zu 99,95\% überein. 


\subsection{Vergleichende Ergebnisse der MRT und der RDC/TMD}

Zum Ende hin gilt es jetzt noch die Ergebnisse der RDC/TMD mit denen der real-time-MRT zu vergleichen. Dafür stehen die drei Befunde „keine Diskusverlagerung (kDV)“, „Diskusverlagerung mit Reposition (DVR)“ und „Diskusverlagerung ohne Reposition (DV)“ zur Verfügung. Aus Gründen der besseren statistischen Auswertbarkeit wurden die Einzelbefunde mit den Zahlen 1 und 0 codiert. Die 1 steht dabei dafür, dass der angegebene Befund festgestellt werden konnte. Die 0 steht im Gegensatz zur 1 dafür, dass der Befund nicht festgestellt werden konnte. Die Ergebnisse sind in der Tabelle 17 aufgeführt. Die Übereinstimmungen zwischen den Ergebnissen der real-time-MRT und den Ergebnissen der RDC/TMD wurden mit einem „,+“ - Zeichen markiert. Alle Fälle, bei denen es nicht zu einer Übereinstimmung kam, wurden mit einem ,,-“Zeichen markiert.

\begin{tabular}{l} 
Vergleich der Ergebnisse des real-time-MRT und deren Nachuntersuchung \\
Nr. \\
\hline
\end{tabular}




\begin{tabular}{|c|c|c|c|c|c|c|c|c|c|c|c|}
\hline 24 & 5538 & rechts & 0 & 1 & - & 1 & 1 & + & 0 & 0 & + \\
\hline 25 & 5539 & rechts & 1 & 1 & + & 0 & 0 & + & 0 & 0 & + \\
\hline 26 & 5560 & rechts & 1 & 1 & + & 0 & 0 & + & 0 & 0 & + \\
\hline 27 & 5561 & rechts & 1 & 1 & + & 0 & 0 & + & 0 & 0 & + \\
\hline 28 & 5580 & rechts & 0 & 1 & - & 0 & 0 & + & 1 & 0 & - \\
\hline 29 & 5599 & rechts & 1 & 1 & + & 0 & 0 & + & 0 & 0 & + \\
\hline 30 & 5591 & rechts & 1 & 1 & + & 0 & 0 & + & 0 & 0 & + \\
\hline 1 & 5353 & links & 1 & 1 & + & 0 & 0 & + & 0 & 0 & + \\
\hline 2 & 5354 & links & 1 & 1 & + & 0 & 0 & + & 0 & 0 & + \\
\hline 3 & 5355 & links & 0 & 0 & + & 1 & 1 & + & 0 & 0 & + \\
\hline 4 & 5356 & links & 0 & 0 & + & 0 & 1 & - & 0 & 0 & + \\
\hline 5 & 5373 & links & 1 & 1 & + & 0 & 0 & + & 0 & 0 & + \\
\hline 6 & 5374 & links & 0 & 0 & + & 1 & 1 & + & 0 & 0 & + \\
\hline 7 & 5375 & links & 1 & 1 & + & 0 & 0 & + & 0 & 0 & + \\
\hline 8 & 5376 & links & 1 & 1 & + & 1 & 0 & - & 0 & 0 & + \\
\hline 9 & 5377 & links & 0 & 1 & - & 1 & 0 & - & 0 & 0 & + \\
\hline 10 & 5378 & links & 0 & 0 & + & 1 & 1 & + & 0 & 0 & + \\
\hline 11 & 5395 & links & 1 & 1 & + & 0 & 0 & + & 0 & 0 & + \\
\hline 12 & 5396 & links & 0 & 1 & - & 1 & 0 & - & 0 & 0 & + \\
\hline 13 & 5397 & links & 1 & 1 & + & 0 & 0 & + & 0 & 0 & + \\
\hline 14 & 5398 & links & 1 & 1 & + & 0 & 0 & + & 0 & 0 & + \\
\hline 15 & 5411 & links & 1 & 1 & + & 0 & 0 & + & 0 & 0 & + \\
\hline 16 & 5412 & links & 1 & 1 & + & 0 & 0 & + & 0 & 0 & + \\
\hline 17 & 5413 & links & 1 & 1 & + & 0 & 0 & + & 0 & 0 & + \\
\hline 18 & 5414 & links & 1 & 1 & + & 0 & 0 & + & 0 & 0 & + \\
\hline 19 & 5415 & links & 1 & 1 & + & 0 & 0 & + & 0 & 0 & + \\
\hline 20 & 5534 & links & 1 & 1 & + & 0 & 0 & + & 0 & 0 & + \\
\hline 21 & 5535 & links & 0 & 0 & + & 1 & 1 & + & 0 & 0 & + \\
\hline 22 & 5536 & links & 1 & 0 & - & 0 & 0 & + & 0 & 0 & + \\
\hline 23 & 5537 & links & 1 & 1 & + & 0 & 0 & + & 0 & 0 & + \\
\hline 24 & 5538 & links & 0 & 1 & - & 1 & 1 & + & 0 & 0 & + \\
\hline 25 & 5539 & links & 0 & 1 & - & 1 & 1 & + & 0 & 0 & + \\
\hline 26 & 5560 & links & 0 & 1 & - & 1 & 0 & - & 0 & 0 & + \\
\hline 27 & 5561 & links & 1 & 1 & + & 0 & 0 & + & 0 & 0 & + \\
\hline 28 & 5580 & links & 0 & 1 & - & 1 & 0 & - & 0 & 0 & + \\
\hline 29 & 5599 & links & 1 & 1 & + & 0 & 0 & + & 0 & 0 & + \\
\hline 30 & 5591 & links & 1 & 1 & + & 0 & 0 & + & 0 & 0 & + \\
\hline
\end{tabular}

Tab.: 20 Vergleich der Ergebnisse der real-time-MRT und der RDC/TMD. kDV = keine Diskusverlagerung, DVR = Diskusverlagerung mit Reposition, DV = Diskusverlagerung ohne Reposition, 1 = Ja, 2 = Nein, ,„“ = keine Übereinstimmung zwischen MRT und RDC/TMD, „+“= Übereinstimmung zwischen MRT und RDC/TMD.

Bei prozentualer Betrachtung lässt sich feststellen, dass der Befund keine Diskusverlagerung bei 49 der 60 Kiefergelenke übereinstimmt, das sind 82\% der untersuchten Fälle. Für die Diskusverlagerung mit Reposition ergibt sich eine prozentuale Übereinstimmung von $87 \%$. Das heißt bei 52 von 60 Kiefergelenken ist der Befund der gleiche. 
Bei der Diskusverlagerung ohne Reposition beträgt der Wert für die prozentuale Übereinstimmung $98 \%$, also die Befunde von 59 der 60 Kiefergelenke stimmen hier überein.

Außerdem ließen sich die Ergebnisse auch auf deren Spezifität und Sensitivität hin untersuchen. Dabei war es notwendig, die RDC/TMD als die etablierte Methode gegen die neue realtime-MRT zu testen.

Vierfeldertafel zur Diskusverlagerung mit Reposition:

\begin{tabular}{|c|c|c|c|}
\hline \multicolumn{2}{|c|}{ DVR } & \multicolumn{2}{c|}{ RDC/TMD } \\
\cline { 3 - 4 } \multicolumn{2}{|c|}{} & P.k./1 & P.g./0 \\
\hline \multirow{2}{*}{ MRT } & T.p./1 & 8 & 6 \\
\cline { 2 - 2 } & T.n./0 & 4 & 42 \\
\hline
\end{tabular}

Sensitivität: $8 /(8+4)=0,67$

Bei 67\% der Probanden mit einer Diskusverlagerung mit Reposition ist die Untersuchung positiv.

Spezifität: 42 / $(42+6)=0,88$

Bei 88\% der Probanden mit normaler Diskusposition ist die Untersuchung negativ.

Vierfeldertafel zur Diskusverlagerung ohne Reposition:

\begin{tabular}{|c|c|c|c|}
\hline \multicolumn{2}{|c|}{ DV } & \multicolumn{2}{c|}{ RDC/TMD } \\
\cline { 3 - 4 } & & P.k./1 & P.g./0 \\
\hline \multirow{2}{*}{ MRT } & T.p./1 & 0 & 1 \\
\cline { 2 - 3 } & T.n./0 & 0 & 59 \\
\hline \multirow{2}{*}{$\begin{array}{c}\text { Tab.: 22 Vierfeldertafel DV : P.g./0 = Patient gesund, P.k./1 = Pati- } \\
\text { ent krank, T.n/0. = Test negativ, T.p/1. = Test positiv }\end{array}$}
\end{tabular}


Sensitivität: $0 /(0+0)=$ nicht definiert

In diesem Fall ist die Sensitivität aus mathematischen Gründen nicht zu berechnen.

Spezifität: $59 /(59+1)=0,98$

Bei 98\% der Probanden mit normaler Diskusposition ist die Untersuchung negativ. 


\section{Diskussion}

\subsection{Validität der RDC/TMD-Untersuchung}

Betrachtet man die durchgeführten Untersuchungen und die daraus gewonnenen Ergebnisse, werden in der vorliegenden Arbeit ausschließlich Befunde der Achse I der RDC/TMD untersucht. Auf die Achse II mit den in diesem Zusammenhang diagnostizierten Schmerzen wird deshalb nicht weiter eingegangen. Eine vergleichbare getrennte Betrachtung der RDC/TMD findet sich auch in der Literatur wieder (Look et al. 2010; Truelove et al. 2010; Schiffman et al. 2010 b). Im Mittelpunkt steht dabei trotz der weiten Verbreitung und der häufigen Anwendung der RDC/TMD deren klinische Kompatibilität. Außerdem tauchen in der Literatur immer wieder kritische Fragen auf, die sich insbesondere auf die diagnostischen Bewertungsalgorithmen für myofasziale Schmerzen und die Diskusverlagerungen mit Reposition beziehen (Look et al. 2010; Schiffman et al. 2010 b). Überdies werden die Ausführbarkeit aller palpatorischen Untersuchungen an den vorgesehenen Stellen und die Möglichkeiten, die RDC/TMD in den klinischen Alltag einzubauen, durchaus kritisch bewertet (Naeije et al. 2009; Steenks und de Wijer 2009).

Außerdem wurde versucht, die RDC/TMD auf ihre praktische und klinische Anwendbarkeit zu untersuchen. Dabei ergab sich unter anderem, dass gewisse Untersuchungspunkte für die abschließende Diagnose stärker oder schwächer ins Gewicht fallen und damit ihre Erhebung wichtiger oder unwichtiger wird. Es sollte deshalb in Erwägung gezogen werden, welche Punkte vernachlässigt werden können, damit die praktische und klinische Anwendbarkeit erhöht wird (Schmitter et al. 2008).

Diese Mängel bei der Anwendung der RDC/TMD sind auch deren Befürwortern nicht verborgen geblieben, und so wurden noch im Jahre 2010 sechs zusammenhängende Artikel veröffentlicht, welche sich alle mit der Validität der RDC/TMD beschäftigten und zusammengefasst als Validität-Projekt der RDC/TMD bezeichnet werden (Anderson et al. 2010; Look et al. 2010; Ohrbach et al. 2010; Truelove et al. 2010; Schiffman et al. 2010 a; Schiffman et al. 2010 b). In sämtlichen Artikeln werden die über Jahre hinweg gesammelten praktischen Erfahrungen zur Anwendung der RDC/TMD sowie die Auffassungen der Autoren und deren Nachweis durch wissenschaftliche Studien dazu herangezogen, die Grundlagen der RDC/TMD zu hinterfragen, deren Nutzen zu analysieren und Verbesserungen für die Zukunft 
vorzuschlagen (Schiffman et al. 2010 a). Schiffman et al. (2010) führen in ihrer Veröffentlichung weiter ins Feld, dass die RDC/TMD nur eine Kopie dessen sei, was sowieso in der Klinik bei der Behandlung von CMD-Patienten gemacht werde, und ferner, dass sie von Spezialisten auf dem Gebiet der CMD entwickelt worden seien, um die Behandlung der CMD zu vereinheitlichen und evidenzbasiert zu gestalten (Schiffman et al. 2010 a).

Die zweite Studie des Validität-Projektes befasst sich mit der Diagnosefindung entlang der ersten Achse der RDC/TMD. Diese ist von besonderer Relevanz für die vorliegende Untersuchung, da sich die erste Achse der RDC/TMD unter anderem mit den speziellen Pathologien

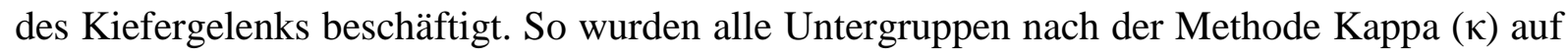
die zwischengutachterliche Reliabilität geprüft. Für myofaciale Schmerzen $(\kappa=0,62)$ und myofasziale Schmerzen mit limitierter Mundöffnung $(\kappa=0,58)$ zeigen die $\kappa$-Werte eine gute Reliabilität. Wurde die gesamte Gruppe I zusammengefasst, war der $\kappa$-Wert $(\kappa>75)$ sogar noch höher. Für die Diskusverlagerung mit Reposition $(\kappa=0,63)$, die Diskusverlagerung ohne Reposition mit limitierter Mundöffnung $(\kappa=0,62)$ und die Diskusverlagerung ohne Reposition und ohne limitierte Mundöffnung $(\kappa=0,31-0,41)$ ergaben sich die angegebenen Werte (Look et al. 2010). Für die beiden ersten häufiger auftretenden Formen der Diskusverlagerung sind die Werte als sehr gut zu bezeichnen, während der Wert für die seltener auftretende Diskusverlagerung ohne Reposition und ohne limitierte Mundöffnung als wenig aussagekräftig $\mathrm{zu}$ bewerten ist. Auch die Gruppe III wurde mit $\kappa$ bewertet. Hierbei schneidet die Arthralgie $(\kappa=0,55)$ noch relativ gut ab, dagegen die Osteoarthritis $(\kappa=0.31-0,43)$ nur schlecht (Look et al. 2010). Im Ergebnis stellt diese Studie damit zutreffend heraus, dass die RDC/TMD für die Diagnostik von myofaszialen Schmerzen, Diskusverlagerungen mit Reposition bzw. ohne Reposition, aber mit limitierter Mundöffnung und Arthralgien sehr gut geeignet sind. Dies scheint einerseits darin begründet zu sein, dass diese Pathologien mit am häufigsten vorkommen und daher die Gutachter auch die größere Übung darin haben, sie zu diagnostizieren. Andererseits sind sie aber durch ihren einfachen Pathomechanismus klinisch besser zu verifizieren. Folglich erscheint die Annahme durchaus gerechtfertigt, dass die $\kappa$-Werte für die Diskusverlagerung ohne Reposition (Anderson et al. 2010) und ohne limitierte Mundöffnung sowie für die Osteoarthritis schlecht sind. Derartige Pathologien sind im Kiefergelenk eher von seltenerer Natur und haben einen hochdifferenzierten Pathomechanismus, welcher eine genauere Diagnostik ohne bildgebende Verfahren nur schwer möglich macht. Damit verdeutlicht diese Studie aber auch, dass die RDC/TMD durchaus dazu geeignet und in der Lage sind, CMD-Patienten bei ersten klinischen Untersuchungen sinnvoll einzuordnen und als Konse- 
quenz daraus bei einem bestehenden Verdacht auf schwerwiegendere Pathologien weitere Mittel zur Diagnosesicherung heranzuziehen.

Dieses Ergebnis wird auch in einer weiteren Studie des Validität-Projektes beschrieben (Truelove et al. 2010). Dabei wurden Patienten entlang der Vorgaben der RDC/TMD klassifiziert. Die Klassifizierung wurde von Dental-Hygienikern/-innen vorgenommen, welche zuvor in die RDC/TMD eingewiesen wurden. Die Ergebnisse wurden danach mit einem „Goldstandard" verglichen. Dieser wurde von zwei Experten auf dem Gebiet der CMD, denen alle Unterlagen der Patienten zur Verfügung standen, also neben allen klinischen Untersuchungen auch das bildgebende Material, festgelegt (Truelove et al. 2010). Die RDC/TMD wurden danach auf Sensitivität und Spezifität im Vergleich zu den Ergebnissen des „Goldstandard” bewertet. Dabei war die angestrebte Sensitivität 0,70 und die angestrebte Spezifität 0,95. Nun wurden für Diagnosen aus der Gruppe I eine Sensitivität von 0,87 und eine Spezifität von 0,98 erreicht. Für die Diagnosen der Gruppe II und III ergab sich jedoch nur eine Sensitivität von 0,03 - 0,53 und eine Spezifität von 0,86 - 0,99 (Truelove et al. 2010). Nach Ansicht der Autoren bedarf es daher einer Überarbeitung der Bewertungsalgorithmen der Achse I, um die Validität der RDC/TMD gerade im Bereich der Diskusverlagerung zu erhöhen und damit sicherer zu machen. Dieses Ergebnis zeigt damit sehr deutlich, dass die RDC/TMD allein als diagnostisches Mittel nicht ausreichen, um, wie hier gezeigt wurde, Patienten mit Diskusverlagerungen sicher zu diagnostizieren und den einzelnen Untergruppen zuzuordnen. Dabei ist $\mathrm{zu}$ berücksichtigen, dass in dieser Studie die RDC/TMD nur von geschulten DentalHygienikern/-innen durchgeführt wurde, bei denen jedoch keine nennenswerte Erfahrung in der Behandlung und Diagnostik des Krankheitsbildes CMD beschrieben wurde. Gleichwohl unterstreicht die Arbeit damit den diagnostischen Nutzen von bildgebenden Verfahren, sei es nun die MRT bei Diskusverlagerung oder sei es die CT bei pathologischen Veränderungen des Knochens. Dies spricht für die Anwendung einer kombinierten Verfahrensweise. Zunächst können die Patienten mittels RDC/TMD in die jeweiligen diagnostischen Gruppen eingeteilt werden. Danach sollte mittels bildgebender Verfahren in einschlägigen Fällen eine weitere Diagnostik betrieben werden, in denen aufgrund der geringen Sensitivität der RDC/TMD keine eindeutige Diagnose gestellt werden konnte.

Weiterhin äußerten sich Anderson et al. (2010) in ihrem Artikel zu zahlreichen sich in diesem Zusammenhang stellenden Fragen. Sie beschreiben die in der Zukunft möglichen und/oder notwendigen Änderungen und Verbesserungen der RDC/TMD (Anderson et al. 2010). Dazu werden diverse Themen angesprochen. Eindeutig scheint hierbei zu sein, dass die Anzahl der 
an dem Validität-Projekt beteiligten Patienten bei weitem nicht ausreicht, um qualifizierte Aussagen über die weniger häufigen Untergruppen der RDC/TMD treffen zu können. Ferner führen Anderson et al. (2010) ins Feld, dass weitere Untergruppen unumgänglich seien, da die individuelle Ausprägung einer CMD bei jedem Patienten unterschiedlich oder zum Teil mit anderen aus anatomischer Sicht benachbarten Regionen vergesellschaftet sei. Sie diskutieren ferner, ob die CMD Auslöser von Schmerzen in diesen Regionen sein könne. In diesem Zusammenhang sprechen sie z.B. den Spannungskopfschmerz an. Dieser könnte aber ohne weiteres als Untergruppe I.c. in den RDC/TMD seinen Platz finden. Sie fordern zudem, dass die bildgebende Diagnostik Einzug in die RDC/TMD halten sollte. Dies soll dort geschehen, wo eine ausreichende Sensitivität und Spezifität nicht gewährleistet werden könnte. Auf dieser Linie liegen auch Anderson et al. (2010) die daran zweifelten, ob die von Dworkin und LeResche (1992) zur RDC/TMD angestrebte Sensitivität von 0,7 und Spezifität von 0,95 überhaupt erreichbar ist (Anderson et al. 2010).

Schon der Erstautor der RDC/TMD, S. F. Dworkin, wies bei der Erstveröffentlichung der RDC/TMD bereits darauf hin, dass diese einem Entwicklungsprozess unterliegen werde und auch werden müsse, um mit den Entwicklungen der Zeit Schritt halten zu können. Daher hat er ganz aktuell im Jahre 2010 zu dem derzeitigen Stand der Entwicklung sowie zu den in Zukunft geplanten Überarbeitungen und im Speziellen zum Validität-Projekt Stellung genommen (Dworkin 2010). Dabei ist ihm durchaus bewusst, dass die RDC/TMD auf Grund von neueren Erkenntnissen noch nicht den für ein einfaches Diagnose-System für CMD-Patienten erforderlichen Standard haben. Er begrüßt daher ausdrücklich den derzeitigen Entwicklungsprozess. Sehr deutlich stellt er dazu heraus, in welchen Punkten die RDC/TMD verbessert werden können:

- Er teilt die Ansicht von Anderson et al. (2010), dass der Name „research” nicht mehr adäquat sei, da die RDC/TMD bereits Einzug in den klinischen Alltag gehalten haben und noch stärker halten sollten. Neuester Stand sei, dass eine für den klinischen Alltag bestimmte Diagnostic Criteria for Temporomandibular Disorders (DC/TMD) herausgegeben werden sollte. Diese sollte Verbesserungen zum klinischen Alltag und zu oft gestellten Diagnosen innerhalb der RDC/TMD enthalten. Außerdem sei eine Weiterentwicklung der RDC/TMD geplant, jedoch wird die Herausgabe wegen größerer Untersuchungen noch längere Zeit in Anspruch nehmen. (List und Greene 2010) 
- Auch stimmt Dworkin (2010) mit Anderson et al. (2010) darin überein, dass eine Reevaluation der Werte für die Spezifität und Sensitivität von Nöten sei. Dabei hält er den Vorschlag von Anderson et al. (2010) für sinnvoll, auf Kosten der Spezifität die Sensitivität zu erhöhen. Er weist aber gleichzeitig darauf hin, dass dies zum Wohle der Patienten mit größter Sorgfalt zu geschehen habe.

- Darüber hinaus unterstützt er die von Anderson et al. (2010) vorgeschlagenen Subtypen für die Achse I-Untersuchungen. Dabei teilt er deren Auffassung, dass die von ihm 1992 angewendeten Untersuchungsmethoden zum Teil nicht mehr zeitgemäß seien und dass die Übernahme von neueren Methoden absolut wünschenswert erscheine.

- Schließlich sollten auch Punkte der Achse II reevaluiert werden. Ausführungen dazu erscheinen im Rahmen dieser Arbeit in dessen nicht angezeigt, da sich alle Befunde des real-time-MRT nur auf die Achse I der RDC/TMD beziehen.

Anzumerken ist, dass Dworkin (2010) zur Präsentation der Strategien, wie die erarbeiteten Verbesserungsvorschläge in die Tat umzusetzen sind, nichts schuldig bleibt. Sicherlich ließen sich dazu zeitnah einige Verbesserungen einarbeiten. Andere Verbesserungsvorschläge benötigen hingegen eine längere Planung und eine vorausgehende Überprüfung. Weiterhin betont er die harte und gute Arbeit der am Validität-Projekt beteiligten Referenten. Aber er weist zugleich darauf hin, dass eine erfolgsorientierte Zusammenarbeit, die zu verbesserten RDC/TMD führt, nur dann möglich sei, wenn alle Beteiligten objektiv blieben und keine eigenen Interessen verfolgten (Dworkin 2010).

Nach diesen negativen Aspekten und den kritischen Anmerkungen zu den RDC/TMD und den aufgezeigten Diskussionen rund um die RDC/TMD geht es nun darum, ein Fazit zu ziehen. Obwohl die RDC/TMD nach Anderson et al. (2010) die für die Untersuchung selbst geforderte Güte nicht erreicht haben, werden sie doch häufig für wissenschaftliche Studien nutzbar gemacht, so dass sie zur Zeit das weltweit anerkannteste Diagnoseverfahren bei CMD-Patienten sind. Außerdem hat sich gezeigt, dass die RDC/TMD im klinischen Alltag ein probates Mittel darstellen, um CMD-Patienten nach einem einheitlichen System einzuordnen. Dabei zeigt gerade der Entwicklungsprozess, dass das System der RDC/TMD ihren Zweck sehr gut erfüllt und dass sie geeignet sind, als Basis für Weiterentwicklungen zu dienen. Zulässig ist danach die Feststellung, dass die RDC/TMD trotz ihrer Mängel zur Zeit eine 
der besten klinischen Screening-Untersuchungs-Verfahren für CMD-Patienten sind und sich angesichts ihrer anstehenden Weiterentwicklung noch verbessern werden. 


\subsection{Validität der real-time-MRT gegenüber der statischen MRT}

Das Bestreben war immer darauf gerichtet, physiologische oder auch pathologische Vorgänge im menschlichen Körper non-invasiv zu untersuchen (Uecker et al. 2010). Schon 1989 sahen Fulmer und Harms voraus, dass die MRT mit Oberflächenspulen und CINE-Technik zum Standard in der Kiefergelenksdiagnostik werden könnte (Fulmer und Harms 1989). Dabei prognostizierten sie, dass sich gerade die MRT-Forschung stark entwickeln und der Behandler in die Lage versetzt werde, Kiefergelenksbewegungen zu untersuchen, um auf diese Weise eine fehlerhafte Funktion der Kiefergelenke festzustellen.

Bereits 1987 haben Burnett et al. eine Methode vorgeschlagen, die Bewegung des Kiefergelenks mit zu untersuchen. Dies geschah mit einer verstellbaren Aufbisshilfe, mit der eine Serie von Bildern des Kiefergelenks bei verschiedenen Öffnungsgraden des Mundes aufgenommen wurde. Sie prognostizierten, dass die damals noch nicht gegebene Möglichkeit, das Kiefergelenk in Bewegung zu untersuchen, einen Rückschlag bei routinemäßiger Anwendung der MRT zur Untersuchung der Funktion des Kiefergelenks führen könnte (Burnett et al. 1987).

Die hier vorgestellte real-time-MRT spiegelt den heutigen Stand der Technik wider. Sie ist in der Lage, ohne jedwede mechanische Hilfe die Kieferöffnungs- und die Kieferschließbewegung in ihrem tatsächlichen Bewegungsablauf aufzunehmen und wiederzugeben (Zhang et al. 2011).

Selbst so komplexe Vorgänge wie die Entstehung der Sprache konnte mit Hilfe der real-timeMRT dargestellt werden. Dies wäre mit der statischen MRT oder aber auch mit CINEMethoden schlichtweg unmöglich gewesen (Uecker et al. 2010). Sicherlich ist die Kiefergelenksfunktion nicht mit der Komplexität der Sprachbildung zu vergleichen, dennoch sollte man aber die individuellen Bewegungsmuster gerade bei pathologischen Zuständen im Kiefergelenk nicht unterschätzen. Diese sind mit einer statischen oder einer CINE-Technik nicht originalgetreu $\mathrm{zu}$ reproduzieren.

Ähnlich wie bei Ahmad et al. (2009) wurden die Ergebnisse der MRT-Untersuchung, also alle Einzelergebnisse der Gutachter, im Paarvergleich mit Hilfe von Kappa (к) und ferner die gesamte Gruppe mit einer prozentualen Bewertung beschrieben. Dort lagen die $\kappa-W e r t e$ für die MRT bei Diskusverlagerung mit Reposition bei 0,78 und bei Diskusverlagerung ohne Reposition bei 0,94. Die prozentuale Übereinstimmung lag in dieser Studie für die Diskusverlagerung mit und ohne Reposition sowie für den Gelenksbluterguss bei 82\% (Ahmad et al. 2009). 
$\mathrm{Zu}$ ähnlich guten Werten kommen auch Tasaki et al. (1993), Vahlensieck et al. (2002) sowie Lewis et al. (2008) (Tasaki et al. 1993; Vahlensieck et al. 2002; Lewis et al. 2008). Die Werte, die in dieser Arbeit für die entsprechenden Pathologien ermittelt werden konnten, ergeben für die Diskusverlagerung mit Reposition einen $\kappa$-Wert von 0,849. Für die Diskusverlagerung ohne Reposition konnte aus mathematischen Gründen nicht durchweg zwischen allen Gutachtern ein Wert berechnet werden.

Die Werte sind durchweg als sehr gut zu erachten, und im Anschluss an Ahmed et al. (2009) ist festzustellen, dass die MRT, sowohl die statische als auch, wie hier beschrieben, die realtime-MRT, für alle sich stellenden Probleme zur Funktion der Kiefergelenke eine große Hilfe bei der Diagnosefindung darstellt und deshalb mit in die RDC/TMD aufgenommen werden sollte.

Nun könnte man annehmen, dass aus dem Umstand, dass die Werte für die real-time-MRT noch über denen der statischen MRT liegen, dieser ein höherer Informationsgehalt zuzuschreiben ist, weil die Gutachter es mit einem höheren Informationsgehalt leichter haben, Pathologien zu erkennen. Eine solche Annahme ist indes nicht zulässig, da die Probanden bezogen auf eine CMD-Erkrankung alle als gesund bezeichnet werden müssen. So hatte nur einer der Probanden eine Diskusverlagerung ohne Reposition. Dies bedeutet, dass die Ergebnisse nicht unbedingt vergleichbar sind und weitere Untersuchungen mit Patienten, die an einer CMD-Erkrankung leiden, im real-time-MRT erfolgen müssen, um über mehrere Jahre hinweg Daten von Patienten zu sammeln, denen dann eine größere Aussagekraft beizulegen ist. Nun ist es eine realistische Ansicht, dass der real-time-MRT aus den vorgestellten Gründen eine Verbesserung der statischen MRT im Bezug auf die Untersuchung des Kiefergelenks beizumessen ist. Da jedoch das Sammeln und Auswerten der Daten mehrere Jahre in Anspruch nehmen wird, ist ein abschließendes Urteil nur eingeschränkt möglich. Der Ausblick hierauf ist jedoch, was die Anwendung des real-time-MRT im klinischen Alltag angeht, durchaus als positiv anzusehen.

Dies liegt u.a. daran, dass die real-time-MRT mit den üblichen Geräten durchgeführt werden kann. Die Anschaffung weiterer Hardware ist nicht erforderlich. Einzig die Anschaffung für ein bilaterales Spulensystem ist nötig und erscheint zudem für medizinische Zentren finanziell tragbar, zumal dieses nicht allein für die Kiefergelenksuntersuchungen genutzt werden kann, sondern auch z.B. zur Diagnostik des Knie- und Ellenbogengelenks. Überdies ist die einfache und im Vergleich zum statischen MRT kürzere Untersuchung als ein Benefit für den Patienten und für die Auslastung der MRTs an den medizinischen Zentren anzusehen. Brauchte man 
bei einer statischen MRT-Untersuchung noch bis zu 45 Minuten, so können mit der real-timeMRT alle für die Diagnostik notwendigen Aufnahmen innerhalb von 10 Minuten durchgeführt werden. Außerdem könnte auf diese Weise der Schwerpunkt der Untersuchungen in Bezug auf die CMD-Patienten von der klinischen Untersuchung weg und mehr zur bildgebenden Diagnostik verlagert werden, wie dies z.B. von Lewis et al. (2008) gefordert wird (Lewis et al. 2008).

Darüber hinaus ist es den Behandlern schon länger ein Bedürfnis, diagnostische Aussagen über den Moment des Kiefergelenk-Knackens bei CMD-Patienten machen zu können (Klett 1986). Dies war bisher nicht möglich, da nur statische bzw. Serien von statischen Aufnahmen gemacht wurden und die Biomechanik des Kiefergelenk-Knackens nie wirklich betrachtet werden konnte.

Für die genaue Darstellung von funktionell pathologischen Vorgängen ist die MRTUntersuchung in zwei Maximalpositionen unzureichend. Die CINE-MRT-Technik erfüllt bereits die Anforderungen an eine Funktionsdiagnostik und erlaubt somit bereits eine Diagnostik von Bewegungsstörungen (Vogl et al. 1992). Unter Verwendung eines CINE-Programmes können MRT-Aufnahmen zu der jeweiligen Kiefergelenksposition ausgewählt und zu einer kinematographischen Darstellung der Kiefergelenksbewegung zusammengesetzt werden (Vogl et al. 1992). Negativ an der CINE-MRT-Technik ist aber, dass der Mundschluss oft gerade die Serie der Bilder ist, die dann rückwärts abgespielt wird. Damit entspricht sie also nicht der tatsächlichen Schließbewegung und sollte deshalb nicht beurteilt werden. Hinzu kommt noch, dass bei der CINE-Technik nicht dargestellt werden kann, wie sich der Diskus bei der Kieferbewegung unter Belastung verhält. Außerdem ist die Bildauflösung bei der CINE-Technik schlechter. Dazu haben Behr et al. (1996) festgestellt, dass sich ein Mehrwert an Informationen nur bei $14 \%$ der Probanden ergibt. Es wurde ausgeschlossen, dass für die Untersuchung einer Diskusverlagerung routinemäßig die statische MRT gemacht werden sollte (Behr et al. 1996). Allerdings ist es, falls sie doch durchgeführt wird, nicht möglich, den Mechanismus der Pathologie zu erkennen; denn dazu ist allein die dynamische Information, egal mit welchem System sie erfasst wurde, von Interesse (Handel et al. 1990; Kordass 1999).

Die MRT des Kiefergelenks erweist sich dabei insofern als hilfreich, als bei einer geplanten Therapie anhand der im MRT gemachten Befunde eine dem jeweiligem Ergebnis angepasste Therapie ausgewählt wird (Vahlensieck et al. 2002). Auch ermöglicht sie dem Radiologen, schon frühzeitig von Kiefergelenksdysfunktionen zu erkennen. Solche sind die Verdickung der lateralen Sehne des M. pterigoideus lateralis, zerrissene retrodiskale Bänder und Bluter- 
güsse innerhalb des Kiefergelenks (Tomas et al. 2006). Die Frage ist nun, ob die statische MRT oder die CINE-MRT von ihrer Technik her überhaupt in der Lage sind, eine derartige Vielzahl an Aussagen zu treffen. Dies scheint wegen der technischen Limitationen nicht gegeben zu sein. Führt man sich die Vor- und Nachteile der real-time-MRT noch einmal vor Augen, nämlich:

- geringerer apparativer Aufwand als bei der CINE-Technik,

- geringerer Zeitaufwand als bei der CINE-Technik und statischer Technik, der wiederum zu einer besseren Auslastung der MRTs an den Zentren führt,

- Aufnahmen der Kiefergelenksbewegung unter physiologischen/pathologischen Bedingungen,

- Erfassung der Gesamtheit der Komplexität von Pathomechanismen,

- Anschaffung neuer Hardware nur in geringem Umfang erforderlich,

- Senkung der Belastung für Patienten bei etablierten Untersuchungsprotokollen,

- Auslöser für Fehlfunktionen sind unmittelbar nach der Untersuchung nachvollziehbar,

- der Mehrwert an Informationen gleicht die geringfügig schlechtere Bildqualität aus,

so muss man zu dem Schluss kommen, dass die real-time-MRT der statischen MRT überlegen ist. Weiterhin sprechen nicht nur die guten Ergebnisse für eine zukünftige klinische Anwendung der real-time-MRT sondern auch wirtschaftliche und technische Aspekte, denn neben Validität und Reliabilität haben die Kosten von zusätzlichen Untersuchungen einen entschiedenen Einfluss auf die Häufigkeit ihrer Anwendung (Dahlstrom und Lindvall 1996).

Neben der hier vorgestellten Methodik gab es und gibt es auch eine Reihe von technisch in anderer Weise durchgeführten Verfahren, welche für dynamische MRT-Untersuchungen in Betracht gezogen wurden (Chen et al. 2000; Abolmaali et al. 2004; Azuma et al. 2009). Allerdings haben diese bisher nicht die Bildqualität geliefert, die nötig wäre, um daraus einen 
klinischen Nutzen zu ziehen (Zhang et al. 2011). Unsere Arbeitsgruppe beschreibt, dass sich diese Verfahren im Unterschied zu dem hier vorgestellten System als unterlegen erweisen, was die Auflösung, den Gewebskontrast und die Artefakte angeht (Zhang et al. 2011).

Als Resümee ist festzuhalten, dass die Vorteile des vorgestellten Verfahrens eindeutig überwiegen und die real-time-MRT eine valide Methode zur funktionellen Untersuchung der Kiefergelenke ist. Weitere klinische Studien müssen noch unter Beweis stellen, dass die realtime-MRT außerdem geeignet ist, die statische MRT und auch die CINE-Technik-MRT im klinischen Alltag abzulösen. 


\subsection{Vergleich der Ergebnisse von RDC/TMD und real-time-MRT}

Es hat sich gezeigt, dass sich bei der Behandlung von CMD-Patienten, die allein einer klinischen Untersuchung unterzogen wurden, nicht alle pathologischen Zustände auffinden lassen und deshalb eine hinreichend sichere Diagnose erschwert wird (Marguelles-Bonnet et al. 1995). Dabei ist die MRT-Untersuchung bereits die allgemein akzeptierte Untersuchung bei Vorliegen einer CMD (Emshoff et al. 2002 a). Umso wichtiger ist es, die MRT im klinischen Alltag noch stärker zu etablieren und die bereits gegenwärtigen Vorteile der statischen MRT in der Befunderhebung von CMD-Patienten durch die Anwendung der real-time-MRT noch weiter auszubauen. So sollen im Folgenden noch einmal die Vorzüge der real-time-MRT auch gerade im Vergleich mit den RDC/TMD, also mit einer klinischen Untersuchung, dargestellt werden. Abschließend erscheint es sinnvoll, einen Ausblick darauf zu werfen, ob die realtime-MRT in Zukunft in der Lage sein wird, die statische MRT in der Diagnostik der Kiefergelenkserkrankungen zu ersetzten, und ob die dynamischen Informationen einen Vorteil in der Behandlung von CMD-Patienten erbringen können.

Betrachtet man nun abschließend die Ergebnisse der RDC/TMD mit denen der real-timeMRT, so ergibt sich für den Befund Diskusverlagerung mit Reposition, dass die Befunde in $87 \%$ der untersuchten Fälle überein stimmen. Damit erreicht die real-time-MRT eine Sensitivität von 0,67 und eine Spezifität von 0,88. Prozentual ausgedrückt bedeutet dies, dass die Untersuchung bei $67 \%$ der Kiefergelenke mit einer Diskusverlagerung und Reposition diese auch feststellt und bei $88 \%$ der Kiefergelenke mit physiologischer Diskusposition diese auch als physiologisch ausweist.

Eine weiterer untersuchter Befund trifft Aussagen über die Diskusverlagerung ohne Reposition. Hier stimmten die Befunde in $98 \%$ der untersuchten Fälle überein. Leider ließ sich die Sensitivität aus mathematischen Gründen nicht berechnen, da in der Formel für die Berechnung der Sensitivität Null als Divisor auftaucht. Dies kommt durch den Umstand zustande, dass in den RDC/TMD diese Pathologie nicht diagnostiziert wurde. Die Spezifität betrug 0,98. Daher lässt sich auch nur die Aussage formulieren, dass bei $98 \%$ der Probanden mit normaler Diskusposition die Untersuchung negativ ist. Die Ergebnisse für die Sensitivität und Spezifität dienen der Überprüfung eines medizinischen Testverfahrens, in diesem Falle der real-timeMRT. Dabei sind die Werte für die Sensitivität mit 0,67 gut für einen medizinischen Test, genauso wie die Werte für die Spezifität mit 0,98 und 0,88. Bei einer abschließenden Beurteilung dieser Werte müssen allerdings die Eigenschaften der Gruppe von Probanden unbedingt 
Berücksichtigung finden, damit man aus den Ergebnissen auch die richtigen Schlussfolgerungen zieht.

Im Hinblick darauf, dass es nur Probanden ohne CMD-Historie gab, ist die vorliegende Arbeit nur geeignet, als Grundlage für kommende Untersuchungen zu dienen und eine Prognose für künftige Entwicklungen zu treffen. Gerechtfertigt ist deshalb die Annahme, dass die realtime-MRT der Kiefergelenke in der Lage ist, anatomische Strukturen korrekt wiederzugeben. Auch Pathologien, welche nur bei einigen der Probanden vorhanden waren, wurden korrekt dargestellt. So konnten die Diskusverlagerungen mit Kiefergelenksknacken, welches in einer klinischen Untersuchung einwandfrei zu diagnostizieren ist, auch im real-time-MRT erkannt werden. Die guten Ergebnisse bei den schwerer wiegenden Pathologien lassen sich nicht so leicht darstellen, da lediglich ein Proband eine solche Pathologie aufwies. Demgegenüber machte das Fehlen einer CMD-Historie unter den Probanden es möglich, die RDC/TMD als „Goldstandard“ bei Sensitivität und Spezifität einzusetzen. Schmitter et al. benutzten in ihrer Studie die statische MRT und die RDC/TMD jeweils als „Goldstandard“, um diese Methoden dann mit der Panoramaschichtaufnahme zu vergleichen. Dabei sollte die Form des Kondylus untersucht werden. Es zeigte sich, dass die Werte für Sensitivität beim MRT bei 0,94 und bei den RDC/TMD bei 0,86 lagen. Die Werte für die Spezifität lagen beim MRT bei 0,45 und bei den RDC/TMD bei 0,49. Dies zeigt, dass man mit Hilfe der RDC/TMD durchaus in der Lage ist, aussagekräftige Befunde festzustellen (Schmitter et al. 2006).

Da es in dieser Arbeit aus den oben genannten Gründen nicht sinnvoll erscheint, die statische MRT mit der real-time-MRT zu vergleichen, blieb ein genormtes klinisches Untersuchungsprotokoll als vergleichender Standard in dieser Arbeit als einzige Möglichkeit übrig, andererseits natürlich auch, weil die RDC/TMD eine der gebräuchlichsten ScreeningUntersuchungen ist. Außerdem wird bei CMD-Patienten eine MRT-Untersuchung nicht routinemäßig durchgeführt. Würde man die real-time-MRT direkt mit der statischen MRT vergleichen, müsste man die aus vielen Einzelbildern (180) zusammengesetzte real-time-Aufnahme einfach nur einzeln betrachten. Dabei gingen aber sämtliche aus den Videosequenzen gewonnen Information über die Dynamik der Kiefergelenksbewegung verloren, so dass dies nicht sinnvoll erscheint. Die Auflösung erweist sich bei einem statischen MRT als höher, da eine längere Zeitspanne von 2-3 sec im Gegensatz zur real-time-MRT zur akquirieren der Daten genutzt werden kann. Ob dies aber im Umkehrschluss auch eine Relevanz für die gestellten Befunde hat, ist zu bezweifeln, denn die Auflösung ist nur einer von vielen Faktoren für die Bildqualität und bei einem dynamischen Bewegung ist auch die dynamische Information ent- 
scheidend. Ein weiterer Grund, die RDC/TMD als „Goldstandard“ zu verwenden war, dass bei Patienten ohne CMD-Historie die Diagnostik entlang der RDC/TMD wesentlich leichter ist und $\mathrm{zu}$ sicheren Ergebnissen führt. Bei schwerwiegenden Pathologien, wie z.B. in der Gruppe III der Achse I der RDC/TMD, ist dies indessen nicht gewährleistet.

Zukünftig, wird es darauf ankommen, ob Patienten, die sich mit einer CMD-Symptomatik vorstellen, für die real-time Untersuchung gewonnen werden können. Dies gelingt teilweise schon, doch hat sich gezeigt, dass es noch einige Zeit dauern wird, bis auch eine ausreichende Zahl an Patienten mit einer aussagekräftigen Symptomatik untersucht wurde.

In den RDC/TMD ist bis jetzt kein bildgebendes Verfahren erforderlich, um eine Diagnose zu stellen. Diese erscheint aber auch nur dann sinnvoll, wenn ihr Nutzen gewährleistet ist. Da aber Nachforschungen gezeigt haben, dass bildgebende Untersuchungen, also auch die MRT, nicht immer die Ergebnisse der RDC/TMD stützen, ist die Verbesserung und Abstimmung auf die jeweils andere Untersuchung von entscheidender Bedeutung für zukünftig besser funktionierende Untersuchungen. Dabei sollten indessen bildgebende Verfahren nur dann angewandt werden, wenn deren Nutzen für den Patienten gewährleistet werden kann (Petersson 2010). So muss es ein nachhaltiges Anliegen sein, klinische Untersuchungen mit bildgebenden Verfahren zu kombinieren. Die ersten real-time-MRT-Ergebnisse unserer Arbeitsgruppe zeigen vielversprechende Ergebnisse und könnten dabei in Zukunft von großer Relevanz sein. Sie ermöglicht dem Behandler, schnell und sicher dynamische Informationen über die Vorgänge in den Kiefergelenken zu erhalten und sie in klinische Untersuchungen mit einzubeziehen. Anzumerken ist indessen, dass die real-time-MRT die klinischen Untersuchungen nur ergänzen, aber nicht ersetzen kann. Allerdings liefert die MRT-Untersuchung keine Informationen beispielsweise über Schmerzqualitäten, druckdolente Muskeln und den Verlauf der Scherzsymptomatik. Überdies könnte ein zukünftiges Anwendungsgebiet der real-time-MRT die Kontrolle der Schienentherapie bei CMD-Patienten sein. Die Schienentherapie entweder mit einer Unterkieferäquilibrierungsschiene oder einer bimaxillären Kalottenschiene ist die gebräuchlichste Therapieform bei CMD-Patienten. Bisher erfolgte deren Kontrolle jedoch nur rein okklusal und symptomatisch. Eine Kontrolle der im Kiefergelenk stattfindenden Prozesse gib es schlichtweg nicht. Dieser Mangel könnte durch die realtime-MRT behoben werden, da man die Kieferöffnung und den Kieferschluss sowohl mit und ohne Schiene direkt beobachten kann. Daneben wären Verlaufskontrollen möglich.

Abschließend lässt sich festhalten, dass die real-time-MRT-Ergebnisse unserer Arbeitsgruppe vielversprechend sind und ein Zusammenwirken von RDC/TMD und real-time-MRT wegen 
der Synergieeffekte für zukünftige Untersuchungen von CMD-Patienten wünschenswert erscheint, vorausgesetzt jedoch, dass deren Symptomatik und klinische Untersuchung nicht die nötigen Aufschlüsse über eine adäquate Behandlung ergeben. 


\section{Zusammenfassung}

Im menschlichen Kiefergelenk kann eine störungsfreie Funktion nur dann gewährleistet werden, wenn das Zusammenspiel der anatomischen Strukturen und verschiedenen Regelkreise reibungslos funktioniert. Daher verwundert es nicht, dass die pathologischen Zustände bei CMD-Patienten sehr komplex sind.

Daher ist es nur konsequent, dass die derzeit bestehenden Untersuchungsmethoden hinterfragt, dem wissenschaftlichen Standard angepasst und weiterentwickelt werden. Dies gilt im besonderen Maße für die RDC/TMD, die viele Jahre erfolgreich angewandt wurden und nun intensiv überarbeitet werden, um sie für künftige Aufgaben zu rüsten.

Ebenso verhält es sich mit Untersuchungsmethoden wie der MRT, die ständig durch technische Neuerungen an Aussagekraft gewinnt. Dies gilt gerade auch für die real-time-MRT, die in dieser Arbeit durchaus den Nachweis erbracht hat, dass ihr Einsatz bei der Behandlung von CMD-Patienten regelmäßig zu guten Ergebnissen führt. Diese konnten in der vorliegenden Arbeit durch unsere Arbeitsgruppe klar herausgestellt und mit guten, wissenschaftlich fundierten Ergebnissen unterstrichen werden. Anhand von Spezifität und Sensitivität wurden die Ergebnisse mit konkreten Werten gleichgesetzt und weiter verdeutlicht.

Es wird sich herausstellen, ob die aufgezeigten Vorzüge der real-time-MRT dazu ausreichen, um sie im klinischen Alltag zu etablieren. Die gewonnenen Ergebnisse stehen aber nur am Anfang der Untersuchungen mittels real-time-MRT. Weitere Untersuchungen von CMDPatienten vor oder während der Therapie müssen folgen. Aber bereits diese Ergebnisse haben gezeigt, dass die real-time-MRT im Bereich der Kiefergelenksdiagnostik das Potential besitzt, zu einem im klinischen Alltag angewandten Standard zu werden, und rechtfertigt damit zugleich die neuesten Forschungen.

Zusammenfassend ist festzuhalten, dass der Einsatz der real-time-MRT letztlich dem Wohlergehen der Patienten im gesteigerten Maße zu Gute kommt und aus diesem Grund unbedingt unterstützt und gefördert werden sollte. Neben diesen Aspekten sollten auch die wirtschaftlichen Faktoren Berücksichtigung finden. Auch diese sprechen für die real-time-MRT, da ihre Anwendung nicht nur zu besseren Ergebnissen führt, sondern voraussichtlich sogar mit geringeren Kosten verbunden ist. 


\section{Literaturverzeichnis}

Abolmaali N D, Schmitt J, Schwarz W, Toll D E, Hinterwimmer S und Vogl T J (2004):

Visualization of the articular disk of the temporomandibular joint in near-real-time MRI: feasibility study.

Eur Radiol 14, 1889-1894

Ahmad M, Hollender L, Anderson Q, Kartha K, Ohrbach R, Truelove E L, John M T und Schiffman E L (2009):

Research diagnostic criteria for temporomandibular disorders (RDC/TMD): development of image analysis criteria and examiner reliability for image analysis.

Oral Surg Oral Med Oral Pathol Oral Radiol Endod 107, 844-860

Anderson G C, Gonzalez Y M, Ohrbach R, Truelove E L, Sommers E, Look J O und Schiffman E L (2010):

The Research Diagnostic Criteria for Temporomandibular Disorders. VI: future directions. J Orofac Pain $\underline{24}, 79-88$

Azuma T, Ito J, Kutsuki M, Nakai R, Fujita S und Tsutsumi S (2009):

Analysis of the mandibular movement by simultaneous multisection continuous ultrafast MRI. Magn Reson Imaging 27, 423-433

Barghan S, Merrill R und Tetradis S (2010):

Cone beam computed tomography imaging in the evaluation of the temporomandibular joint. J Calif Dent Assoc 38, 33-39

Behr M, Held P, Leibrock A, Fellner C und Handel G (1996):

Diagnostic potential of pseudo-dynamic MRI (CINE mode) for evaluation of internal derangement of the TMJ.

Eur J Radiol 23, 212-215 
Benninghoff und Drenckhahn:

Anatomie - Makroskopische Anatomie, Histologie Embryologie, Zellbiologie.

16. Auflage, Band 1, Urban \& Fischer Verlag, München 2003, 510-518.

Blankestijn J und Boering G (1985):

Posterior dislocation of the temporomandibular disc.

Int J Oral Surg $\underline{14}, 437-443$

Blaustein D I und Scapino R P (1986):

Remodeling of the temporomandibular joint disk and posterior attachment in disk displacement specimens in relation to glycosaminoglycan content.

Plast Reconstr Surg $\underline{78}$, 756-764

Brooks S L, Brand J W, Gibbs S J, Hollender L, Lurie A G, Omnell K A, Westesson P L und White S C (1997):

Imaging of the temporomandibular joint: a position paper of the American Academy of Oral and Maxillofacial Radiology.

Oral Surg Oral Med Oral Pathol Oral Radiol Endod 푸, 609-618

Bumann A und Lotzmann U:

Farbatlanten der Zahnmedizin - Funktionsdiagnostik und Therapieprinzipien.

1. Auflage, Band 12, Georg Thieme Verlag, Stuttgart 2000

Burnett K R, Davis C L und Read J (1987):

Dynamic display of the temporomandibular joint meniscus by using "fast-scan" MR imaging. AJR Am J Roentgenol 149, 959-962

Cairns B E, Sessle B J und Hu J W (1998):

Evidence that excitatory amino acid receptors within the temporomandibular joint region are involved in the reflex activation of the jaw muscles.

J Neurosci $\underline{18}$, 8056-8064 
Carlson G E und Magnusson T:

Behandlung Temporomandibulärer Funktionsstörungen in der Praxis.

1. Auflage, Quintessenz Verlags-GmbH, Berlin 1999

Chen Y J, Gallo L M, Meier D und Palla S (2000):

Dynamic magnetic resonance imaging technique for the study of the temporomandibular joint. J Orofac Pain $\underline{14}, 65-73$

Clark G T (2008):

Classification, causation and treatment of masticatory myogenous pain and dysfunction.

Oral Maxillofac Surg Clin North Am 20, 145-157

Cohen J (1960):

A coefficient of agreement for nominal scales.

Educat Psychol Measurem 20, 37-46

Costen J B (1997):

A syndrome of ear and sinus symptoms dependent upon disturbed function of the temporomandibular joint. 1934.

Ann Otol Rhinol Laryngol 106, 805-819

Dahlstrom L und Lindvall A M (1996):

Assessment of temporomandibular joint disease by panoramic radiography: reliability and validity in relation to tomography.

Dentomaxillofac Radiol 25, 197-201

Dworkin S F (2010):

Research Diagnostic Criteria for Temporomandibular Disorders: current status \& future relevance.

J Oral Rehabil 37, 734-43 
Dworkin S F und LeResche L (1992):

Research diagnostic criteria for temporomandibular disorders: review, criteria, examinations and specifications, critique.

J Craniomandib Disord $\underline{6}, 301-355$

Dworkin S F und LeResche L (2007):

Entire RDC/TMD Booklet

http://www.rdc-tmdinternational.org/LinkClick.aspx?fileticket=sYtOuTKbw4s\%3d\&tabid

$=960 \& \operatorname{mid}=2911,1-29$

Eberhard D, Bantleon H P und Steger W (2000):

Functional magnetic resonance imaging of temporomandibular joint disorders.

Eur J Orthod 22, 489-497

Emshoff R, Brandlmaier I, Bertram S und Rudisch A (2002 a):

Comparing methods for diagnosing temporomandibular joint disk displacement without reduction.

J Am Dent Assoc 133, 442-451

Emshoff R, Jank S, Bertram S, Rudisch A und Bodner G (2002 b):

Disk displacement of the temporomandibular joint: sonography versus MR imaging.

AJR Am J Roentgenol 178, 1557-1562

Fricton J (2007):

Myogenous temporomandibular disorders: diagnostic and management considerations.

Dent Clin North Am $\underline{51}, 61-83$

Fulmer J M und Harms S E (1989):

The temporomandibular joint.

Top Magn Reson Imaging $\underline{1}, 75-84$

Gallagher D M (1986):

Posterior dislocation of the temporomandibular joint meniscus: report of three cases.

J Am Dent Assoc 113, 411-415 


\section{Gwet K L:}

Intrarater Reliability.

In: Wiley Encyclopedia of Clinical Trials, John Wiley \& Sons, New York 2008, 1-13

Handel G, Gerdes M und Rossbach A (1990):

Three-dimensional dynamic imaging of TMJ movements using stereognathography.

Dtsch Zahnarztl Z $\underline{45}$, S82-84

\section{Helkimo M (1974)}

Studies on function and dysfunction of the masticatory system. II. Index for anamnestic and clinical dysfunction and occlusal state.

Sven Tandlak Tidskr $\underline{67}, 101-121$

Hellwig E, Klinek J und Attin T:

Einführung in die Zahnerhaltung

4. Auflage, Urban \& Fischer Verlag, München 2006, 81

Jäger L, Rammelsberg P und Reiser M (2001):

Diagnostic imaging of the normal anatomy of the temporomandibular joint.

Radiologe 41, 734-740

John M, Hirsch C, Reiber T und S D (2006):

Translating the research diagnostic criteria for temporomandibular disorders into German: evaluation of content and process.

J Orofac Pain 20, 43-52,

http://www.rdc-tmdinternational.org

/LinkClick. aspx ?fileticket=FdkWqFHCKIk\%3d\&tabid=978\&mid=2165, 1-21

Katzberg R W, Westesson P L, Tallents R H und Drake C M (1996):

Anatomic disorders of the temporomandibular joint disc in asymptomatic subjects.

J Oral Maxillofac Surg 54, 147-153; discussion 153-145 
Klett R (1986):

Biomechanics of temporomandibular joint clicking. III. Etiology of eccentric and centric disk luxation.

Dtsch Zahnarztl Z $\underline{41}, 684-692$

Kobs G, Bernhardt O, Kocher T und Meyer G (2005):

Critical assessment of temporomandibular joint clicking in diagnosing anterior disc displacement.

Stomatologija $\underline{7}, 28-30$

Kordass B (1999):

The temporomandibular joint in video motion--noninvasive image techniques to present the functional anatomy.

Ann Anat $\underline{181}, 33-36$

Krestan C, Lomoschitz F, Puig S und Robinson S (2001):

Internal derangement of the temporomandibular joint.

Radiologe 41, 741-747

Landis J R und Koch G G (1977):

The measurement of observer agreement for categorical data.

Biometrics $\underline{33}, 159-174$

Larheim T A (2005):

Role of magnetic resonance imaging in the clinical diagnosis of the temporomandibular joint.

Cells Tissues Organs 180, 6-21

Larheim T A, Westesson P und Sano T (2001):

Temporomandibular joint disk displacement: comparison in asymptomatic volunteers and patients.

Radiology 218, 428-432

Lehmann K M und Hellwig E:

Zahnärztliche Propädeutik.

9. Auflage, Urban \& Fischer Verlag, München 2005, 49 
Lewis E L, Dolwick M F, Abramowicz S und Reeder S L (2008):

Contemporary imaging of the temporomandibular joint.

Dent Clin North Am $\underline{52}, 875-890$

List T und Greene C S (2010):

Moving forward with the RDC/TMD.

J Oral Rehabil 37, 731-733

Look J O, John M T, Tai F, Huggins K H, Lenton P A, Truelove E L, Ohrbach R, Anderson G C und Shiffman E L (2010):

The Research Diagnostic Criteria For Temporomandibular Disorders. II: reliability of Axis I diagnoses and selected clinical measures.

J Orofac Pain 24, 25-34

Marguelles-Bonnet R E, Carpentier P, Yung J P, Defrennes D und Pharaboz C (1995):

Clinical diagnosis compared with findings of magnetic resonance imaging in 242 patients with internal derangement of the TMJ.

J Orofac Pain $\underline{9}, 244-253$

Mc Neill C (1997):

History and evolution of TMD concepts.

Oral Surg Oral Med Oral Pathol Oral Radiol Endod $\underline{83}$, 51-60

Meyer G, Bernhardt O und Asselmeyer T (2003):

Moderne Diagnostik- und Therapieformen bei Funktionsstörungen.

Zahnärztliche Nachrichten Niedersachsen ZNN $\underline{10}, 15-22$

Naeije M, Kalaykova S, Visscher C M und Lobbezoo F (2009):

Evaluation of the Research Diagnostic Criteria for Temporomandibular Disorders for the recognition of an anterior disc displacement with reduction.

J Orofac Pain $\underline{23}, 303-311$

Ohrbach R, Turner J A, Sherman J J, Mancl L A, Truelove E L, Schiffman E L und Dworkin S F (2010): 
The Research Diagnostic Criteria for Temporomandibular Disorders. IV: evaluation of psychometric properties of the Axis II measures.

J Orofac Pain $\underline{24}$, 48-62

Ottl P, Reiber T, Lange M und Lauer H-C (2005):

Der klinische Funktionsstatus der Arbeitsgemeinschaft für Funktionsdiagnostik und Therapie in der DGZMK -Arbeitsanleitung-.

http://www.dgzmk.de/uploads/media/Funktionsstatus.pdf

Parsons M T und Boucher L J (1966):

The bilaminar zone of the meniscus.

J Dent Res $\underline{45}, 59-61$

Petersson A (2010):

What you can and cannot see in TMJ imaging - an overview related to the RDC/TMD diagnostic system.

J Oral Rehabil $\underline{37}, 771-778$

Pufe T, Harde V, Petersen W, Goldring M B, Tillmann B und Mentlein R (2004):

Vascular endothelial growth factor (VEGF) induces matrix metalloproteinase expression in immortalized chondrocytes.

J Pathol 202, 367-374

Rohen W J, Yokochi C und Lütjen-Drecoll E:

Fotographischer Atlas der systematischen und topographischen Anatomie - Anatomie des Menschen.

5. Auflage, Schattauer Verlag, Stuttgart 2002, 56

Scapino R P (1983):

Histopathology associated with malposition of the human temporomandibular joint disc.

Oral Surg Oral Med Oral Pathol 55, 382-397

Schiffman E L, Truelove E L, Ohrbach R, Anderson G C, John M T, List T und Look J O (2010 a): 
The Research Diagnostic Criteria for Temporomandibular Disorders. I: overview and methodology for assessment of validity.

J Orofac Pain 24, 7-24

Schiffman E L, Ohrbach R, Truelove E L, Tai F, Anderson G C, Pan W, Gonzalez Y M, John M T, Sommers E, List T, Velly A M, Kang WundLook J O (2010 b):

The Research Diagnostic Criteria for Temporomandibular Disorders. V: methods used to establish and validate revised Axis I diagnostic algorithms.

J Orofac Pain 24, 63-78

Schmitter M, Gabbert O, Ohlmann B, Hassel A, Wolff D, Rammelsberg P und Kress B (2006):

Assessment of the reliability and validity of panoramic imaging for assessment of mandibular condyle morphology using both MRI and clinical examination as the gold standard.

Oral Surg Oral Med Oral Pathol Oral Radiol Endod 102, 220-224

Schmitter M, Kress B, Leckel M, Henschel V, Ohlmann B und Rammelsberg P (2008):

Validity of temporomandibular disorder examination procedures for assessment of temporomandibular joint status.

Am J Orthod Dentofacial Orthop 133, 796-803

Schwenzer N und Ehrenfeld M:

Zahn- Mund- Kieferheilkunde: Zahnärztliche Chirugie.

3.Auflage, Band 3, Georg Thieme Verlag, Stuttgart 2000, 268-278

Steenks M H und De Wijer A (2009):

Validity of the Research Diagnostic Criteria for Temporomandibular Disorders Axis I in clinical and research settings.

J Orofac Pain 23, 9-16; discussion 17-27

Sunakawa M, Chiang C Y, Sessle B J und Hu J W (1999):

Jaw electromyographic activity induced by the application of algesic chemicals to the rat tooth pulp.

Pain $\underline{80}, 493-501$ 
Tanaka E, Detamore M S und Mercuri L G (2008):

Degenerative disorders of the temporomandibular joint: etiology, diagnosis, and treatment.

J Dent Res $\underline{87}$, 296-307

Tasaki M M, Westesson P L und Raubertas R F (1993):

Observer variation in interpretation of magnetic resonance images of the temporomandibular joint.

Oral Surg Oral Med Oral Pathol 트, 231-234

Tomas X, Pomes J, Berenguer J, Quinto L, Nicolau C, Mercader J M und Castro V (2006):

MR imaging of temporomandibular joint dysfunction: a pictorial review.

Radiographics $\underline{26}, 765-781$

Truelove E, Pan W, Look J O, Mancl L A, Ohrbach R K, Velly A M, Huggins K H, Lenton P und Shiffman E L (2010):

The Research Diagnostic Criteria for Temporomandibular Disorders. III: validity of Axis I diagnoses.

J Orofac Pain $\underline{24}, 35-47$

Türp J C und Hugger A (2000):

Schmerzhafte Myoarthopatien des Kausystems.

Zahnärztl Mitt Online 10/2000, 35-42

Uecker M, Zhang S, Voit D, Karaus A, Merboldt K D und Frahm J (2010):

Real-time MRI at a resolution of $20 \mathrm{~ms}$.

NMR Biomed 23, 986-994

Vahlensieck M, Okweschokwu S und Greven M (2002):

Magnetic Resonance Imaging (MRI) of the TMJ: Influence on therapy and inter-observer agreement of two radiologists.

Rofo $\underline{174}, 1415-1421$

Vasconcelos B C, Rocha N S und Cypriano R V (2010):

Posterior dislocation in intact mandibular condyle: an unusual case.

Int J Oral Maxillofac Surg 39, 89-91 
Vogl T J, Eberhard D, Weigl P, Assal J und Randzio J (1992):

The use of the "cine-technic" in the MRT diagnosis of the temporomandibular joint.

Rofo $\underline{156}, 232-237$

Westesson P L (1993):

Reliability and validity of imaging diagnosis of temporomandibular joint disorder.

Adv Dent Res $\underline{7}, 137-151$

Wish-Baratz S, Ring G D, Hiss J, Shatz A und Arensburg B (1993):

The microscopic structure and function of the vascular retrodiscal pad of the human temporomandibular joint.

Arch Oral Biol $\underline{38}, 265-268$

Wong M, Siegrist M und Goodwin K (2003):

Cyclic tensile strain and cyclic hydrostatic pressure differentially regulate expression of hypertrophic markers in primary chondrocytes.

Bone $\underline{33}, 685-693$

Zhang S, Block K T und Frahm J (2010):

Magnetic resonance imaging in real time: advances using radial FLASH.

J Magn Reson Imaging $\underline{31}$, 101-109

Zhang S, Gersdorff N und Frahm J (2011):

Real-Time Magnetic Resonance Imaging of Temporomandibular Joint Dynamics.

Open Med Imaging J, $\underline{4}, 1-9$ 


\section{Abbildungsverzeichnis}

Abb. 1 Anatomie des Kiefergelenks

(modifiziert Rohen et al. 2002)

Abb. 2: Bewegungsmuster Mundöffnung.

(Bumann und Lotzmann 2000)

Abb. 3 Bewegungsmuster Mundschluss

(Bumann und Lotzmann 2000)

Abb.4: Physiologische myogene Funktion

(Meyer et al. 2003)

Abb.5: Pathologische myogene Funktion

(Meyer et al. 2003)

Abb.6: Diskusverlagerung mit totaler Reposition

(Krestan et al. 2001)

Abb.7: Diskusverlagerung ohne Reposition

(Krestan et al. 2001)

Abb. 8: Normale Anatomie MRT

(modifiziert Larheim 2005)

Abb. 9: Exemplarisch aus einer real-time-MRT-Untersuchung ausgewählte

Einzelbilder eines Videos.

Mit freundlicher Genehmigung der Biomedizinischen NMR-Forschungs-

GmbH (Direktor Prof. Dr. Jens Frahm) S.46

Abb. 10: Gruppe: I Muskelerkrankungen S. 48

(Dworkin und LeResche 1992)

Abb. 11: Gruppe: II Verlagerung des Discus articularis. .5 .50

(Dworkin und LeResche 1992)

Abb. 12 Gruppe: III Arthralgie, Arthritis, Arthrose des Kiefergelenks

(Dworkin und LeResche 1992) 


\section{Tabellen-/Diagrammverzeichnis}

\section{Tabellen}

Tab. 1: Einteilung der Pathophysiologien des Kiefergelenks nach Dworkin S.16 (Dworkin und LeResche 1992)

Tab. 2: Dysfunktionsindex nach Helkimo (Helkimo 1974)

Tab. 3: Anamnestische Angaben des Patienten. S.34 - 37 (John et al. 2006)

Tab. 4: Untersuchungsblatt (Achse II Befunde): Schmerzanamnese. S. 38 (John et al. 2006)

Tab. 5: Untersuchungsblatt (Achse II Befunde): Schmerzempfindung. S.38 (John et al. 2006)

Tab. 6: Untersuchungsblatt (Achse II Befunde): Mundöffnungsbewegung S.39 (John et al. 2006)

Tab. 7: Untersuchungsblatt (Achse II Befunde): Vertikaler Bewegungsumfang.......S.39 (John et al. 2006)

Tab. 8: Untersuchungsblatt (Achse II Befunde): Gelenkgeräusche (Palpation)...........S.40 (John et al. 2006)

Tab. 9: Untersuchungsblatt (Achse II Befunde): Bewegungen. S. 40 (John et al. 2006)

Tab. 10: Untersuchungsblatt (Achse II Befunde): Gelenkgeräusche bei Bewegung.....S.41 (John et al. 2006)

Tab. 11: Untersuchungsblatt (Achse II Befunde): Extraorale Muskelpalpation S.42 (John et al. 2006)

Tab. 12: Untersuchungsblatt (Achse II Befunde): Palpation des Gelenkes. S. 42 (John et al. 2006)

Tab. 13: Untersuchungsblatt (Achse II Befunde): Intraorale Palpation S. 42 (John et al. 2006) 
Tab. 14: Spezifikationen für die Aufnahme des Kiefergelenkes mit dem real-time-MRT.

(Zhang et al. 2010)

Tab. 15: Verschlüsselung der Daten der real-time-MRT

Tab. 16: Vierfeldertafel zur Berechnung der Sensitivität und Spezifität .5 .55 (modifiziert Hellwig et al. 2006)

Tab. 17: Ergebnisse der RDC/TMD nach Auswertung der Frage- und Untersuchungsbögen anhand der entsprechenden Bewertungs-Algorithmen. $.5 .56-57$

Tab. 18: Ergebnisse der real-time-MRT Aufnahme nach der Bewertung durch vier Gutachter.

ab.: 19: Vergleich der Ergebnisse der real-time-MRT und deren

Nachuntersuchungen.

Tab.: 20: Vergleich der Ergebnisse der real-time-MRT und der RDC/TMD S.66 - 67

Tab.: 21: Vierfeldertafel kDV .5 .68

Tab.: 22: Vierfeldertafel kDV S.68

\section{Diagramme}

Diagramm 1: Auswertung der RDC/TMD-Untersuchung und Darstellung der Anzahl der gefundenen Diagnosen bezogen auf jedes einzelne Kiefergelenk.

Diagramm 2: Auswertung der Ergebnisse der real-time-MRT Aufnahmen für die Formen der Diskusverlagerungen.

Diagramm 3: Auswertung der Ergebnisse der real-time-MRT-Aufnahmen für den Bewegungsumfang der Kondylen. 


\section{Video-CD:}




\section{Danksagung}

Danken möchte ich dem kürzlich verstorbenen Herrn Prof. Dr. Dr. A. Hüls für die Überlassung des Themas dieser Dissertation.

Mein besonderer Dank gilt Herrn Dr. N. Gersdorff für die Hilfe bei der Durchführung, Planung und für die Übernahme dieser Dissertation.

Danken möchte ich der Abteilung für medizinische Statistik der Universitätsmedizin Göttingen für die fachkundige Beratung.

Auch möchte ich Herrn Prof. Dr. Frahm und Dr. Shuo Zhang für die sehr sympathische Zusammenarbeit und einen tollen Einblick in die MRT-Forschung ganz herzlich danken. 


\section{Lebenslauf}

Am 3.7.1983 wurde ich, Olaf Kling, als zweiter Sohn der Eheleute Dr. Erhard Kling und Elisabeth Kling-Chrysant in Bonn geboren. Ab dem 6. Lebensjahr besuchte ich vier Jahre die St. Laurentius-Grundschule in Bonn-Lessenich und wechselte danach auf das Carl-von-Ossietzky-Gymnasium in Bonn-Ückesdorf. Unterbrochen von einem einjährigen Aufenthalt auf dem Worksop College in Nottinghamshire bestand ich nach meiner Rückkehr aus England im Jahre 2003 das Abitur.

Im Anschluss daran leistete ich als (ausgebildeter) Rettungssanitäter meinen Zivildienst bei der Johanniter-Unfallhilfe in Bonn ab.

Im Jahre 2005 nahm ich mein Studium der Zahnmedizin an der Georg-August- Universität in Göttingen auf und beendete dieses am 25.6.2010 mit dem erfolgreichen Bestehen des Staatsexamens und Erlangen meiner Approbation als Zahnarzt. 